\title{
WestVirginiaUniversity
}

THE RESEARCH REPOSITORY @ WVU

Graduate Theses, Dissertations, and Problem Reports

2020

\section{Modeling and Tuning of CVT Systems for SAE® Baja Vehicles}

\author{
Sean Sebastian Skinner \\ West Virginia University, ssskinner@mix.wvu.edu
}

Follow this and additional works at: https://researchrepository.wvu.edu/etd

Part of the Applied Mechanics Commons

\section{Recommended Citation}

Skinner, Sean Sebastian, "Modeling and Tuning of CVT Systems for SAE® Baja Vehicles" (2020). Graduate Theses, Dissertations, and Problem Reports. 7590.

https://researchrepository.wvu.edu/etd/7590

This Thesis is protected by copyright and/or related rights. It has been brought to you by the The Research Repository @ WVU with permission from the rights-holder(s). You are free to use this Thesis in any way that is permitted by the copyright and related rights legislation that applies to your use. For other uses you must obtain permission from the rights-holder(s) directly, unless additional rights are indicated by a Creative Commons license in the record and/ or on the work itself. This Thesis has been accepted for inclusion in WVU Graduate Theses, Dissertations, and Problem Reports collection by an authorized administrator of The Research Repository @ WVU. For more information, please contact researchrepository@mail.wvu.edu. 


\title{
Modeling and Tuning of CVT Systems for SAE ${ }^{\circledR}$ Baja Vehicles
}

\section{Sean Skinner}

Thesis Submitted to the Benjamin M. Statler College of Engineering and Mineral Resources at West Virginia University

In Partial fulfillment of the requirements for the degree of Master of Science in Mechanical Engineering

Ken Means, Ph.D., Chair

Tony McKenzie, Ph.D.

Terrence Musho, Ph.D.

Department of Mechanical and Aerospace Engineering

\author{
Morgantown, West Virginia
}

2020

Keywords: SAE, Baja, CVT, Tuning, Modeling, Continuously, Variable, Dynamometer Copyright 2020: Sean Skinner 


\begin{abstract}
Modeling and Tuning of CVT Systems for SAE® Baja Vehicles
\end{abstract}

Sean Skinner

Continuously variable transmission (CVT) describes a family of transmission styles. These systems serve the same purpose as their traditional counterparts; transmitting power while offering a variable gearing ratio. The difference lies in the CVT's ability to change ratio continuously between a minimum and maximum without halting power transfer. They offer many benefits of an automatic transmission while being significantly lighter. For this reason, CVTs are often used on $\mathrm{Baja}_{\mathrm{SAE}}{ }^{\circledR}$ vehicles to control gearing ratio.

WVU's Baja team has used mechanical CVTs for many decades. These systems are simpler in the sense that they don't require power to operate. However, understanding the mechanical control scheme they offer can be difficult. Many teams take a trial and error approach to tuning these systems. This consists of altering components to achieve desired outputs. For a mechanically controlled system this process is time consuming and cumbersome. Data collection isn't as effective for future vehicles because gear box ratio, and vehicle weight will disproportionally affect CVT performance.

The purpose of this research is three-fold. One: researching specific, quantifiable, CVT tuning objectives. Areas of consideration include engagement speed, shift out speed, static RPM shifting, and efficient power transfer. Second: developing a model of the CVT force balance. This model is derived from the force balance between the primary and the secondary sub-systems. Third: developing tools to aid in the tuning process. This includes a system testing setup, monitoring power, torque, and shaft speed at the primary and secondary. 
Table of Contents

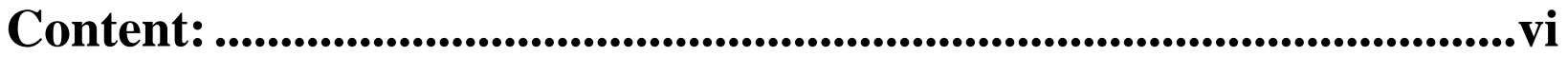

List of Equations: ..................................................................... vi

List of Figures: ................................................................ viii

List of Tables: ...........................................................................

Table of Symbols......................................................................

Acknowledgement: ...............................................................................xi

Introduction: ..............................................................................................1

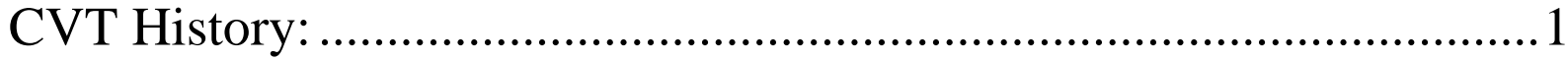

Baja SAE ${ }^{\circledR}$ Application: ................................................................. 3

Importance of Tuning .............................................................. 3

Literature Review: ........................................................................5

System Components and Variables:.................................................6

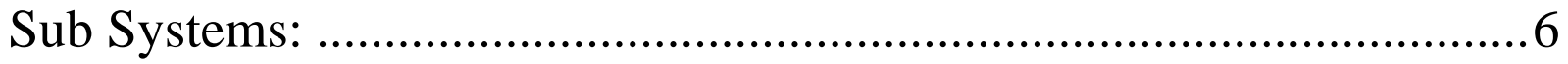

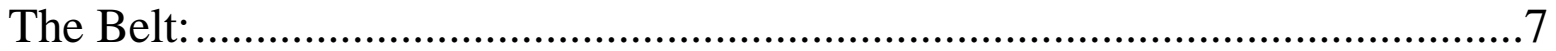

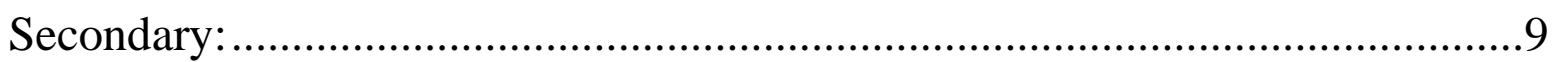

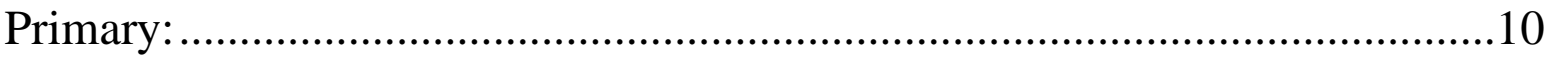

Operating Conditions: ..............................................................12

Clutching Phase: ................................................................... 12

Low Ratio Acceleration: ........................................................... 12

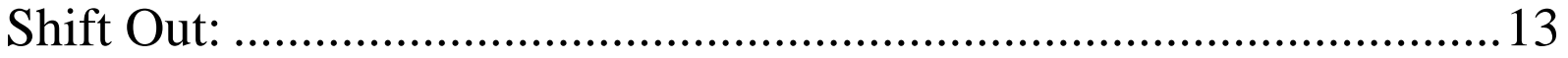

High Ratio Acceleration: ............................................................ 13

Tuning Objectives: ..............................................................................14

Straight Shift:................................................................. 14 


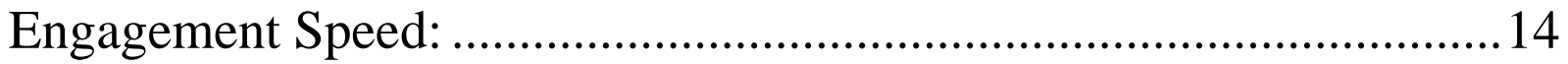

Efficient Power Transfer: ................................................................ 14

Methods: Empirical Analysis and Testing................................................15

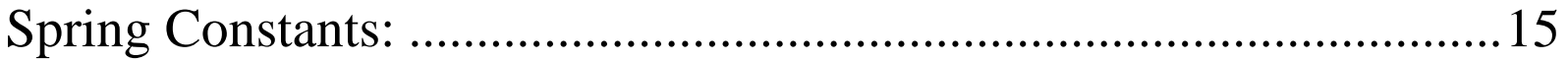

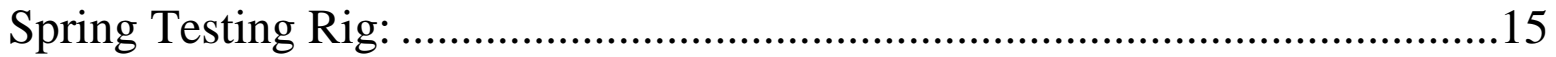

Linear Constants: ........................................................................................

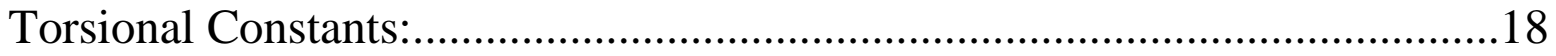

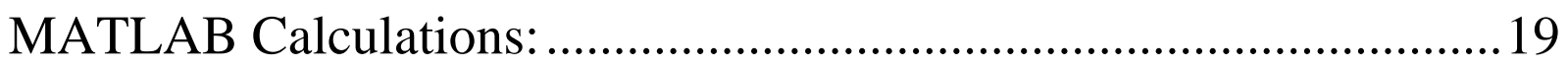

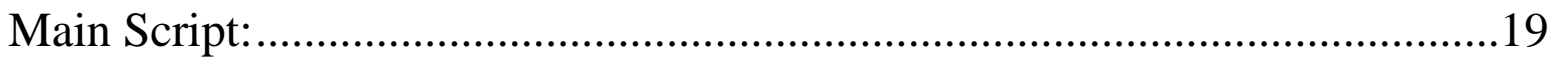

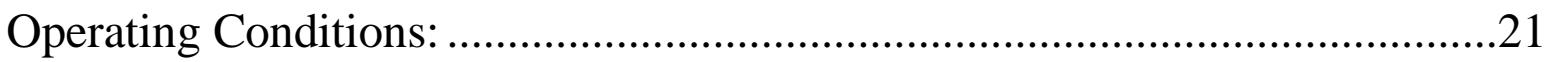

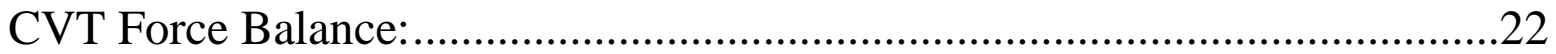

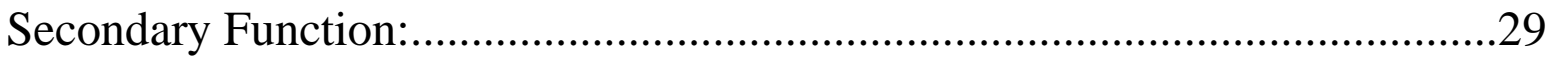

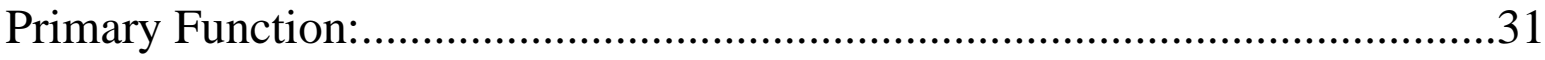

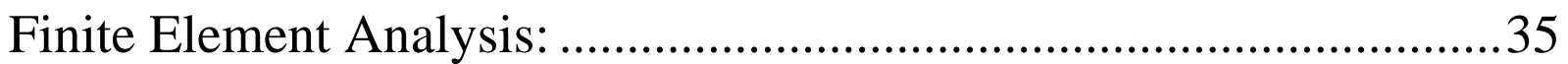

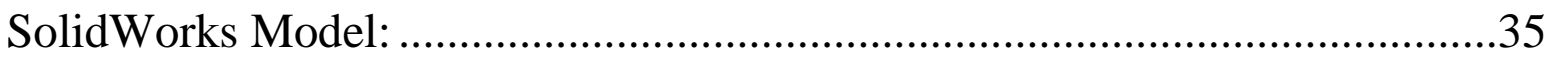

ANSYS Workbench Model: ........................................................................37

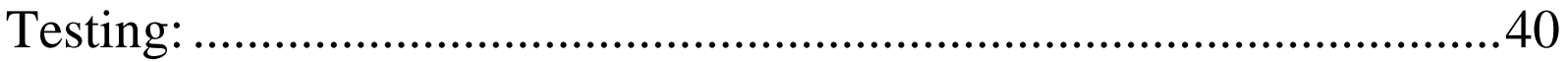

Water-brake Dynamometer ...........................................................................

Data Acquisition Setup: …………………………………………………......4

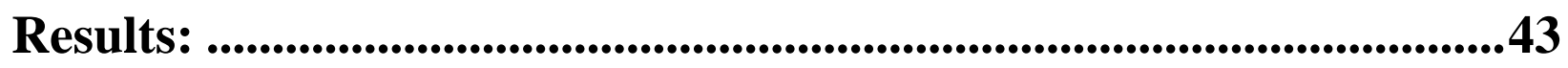

System Constants: ........................................................................4

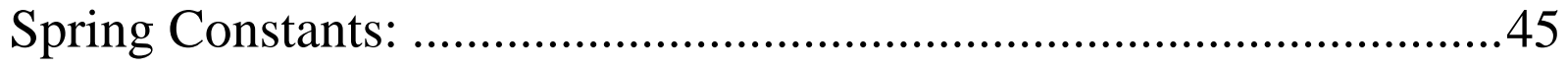

Tunable Component Options: ............................................................4

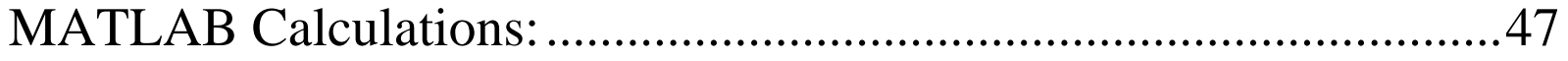

ANSYS Workbench Model:................................................................53

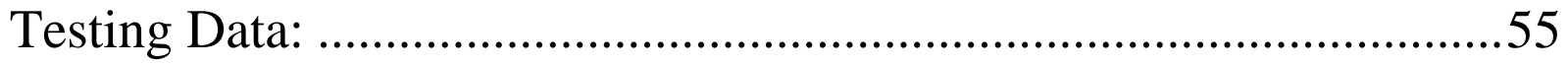


Uncertainty Analysis: .................................................................58

Conclusions: ..........................................................................................55

Contributions: ............................................................................................60

CVT Tuning Research:...........................................................6 60

Spring Testing Rig: ............................................................6 60

CAD Model: ...........................................................................60

MATLAB Setup Analysis:......................................................6 60

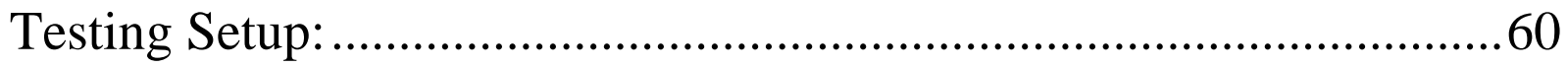

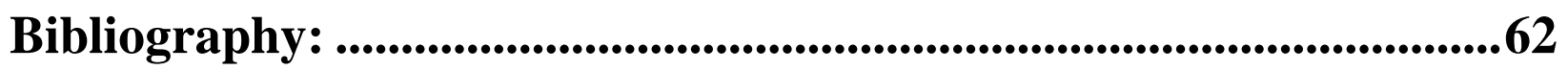

Appendix: .......................................................................................................63

Spring Test Rig Data: .......................................................6 63

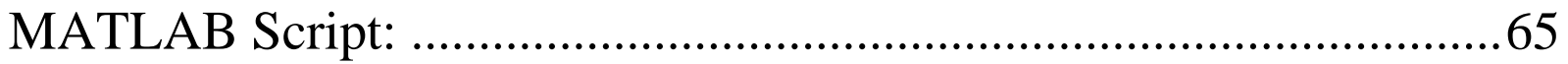

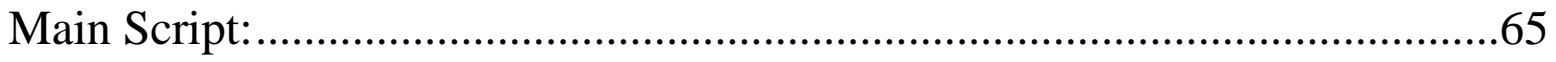

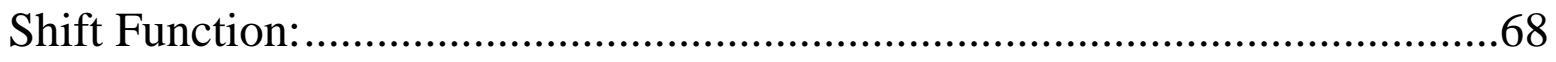

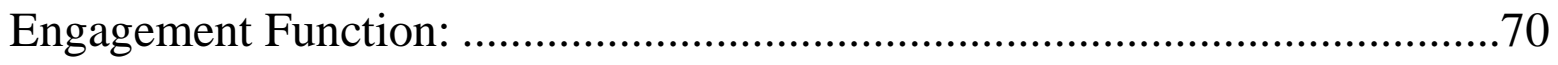

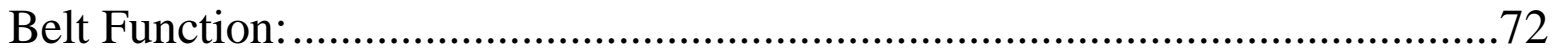

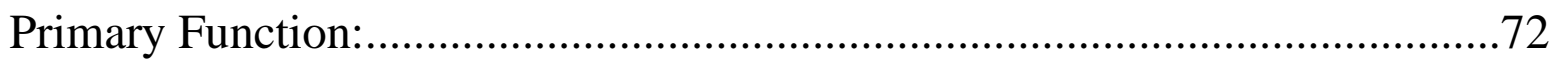

Secondary Function:.............................................................................. 72 


\section{Content:}

\section{List of Equations:}

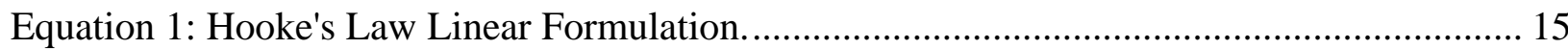

Equation 2: Hooke's Law Torsional Formulation.................................................................... 15

Equation 3: Torsional Spring Constant. (Richard G. Budynas) ……………………………..... 18

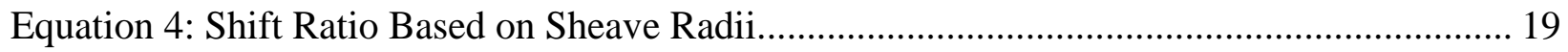

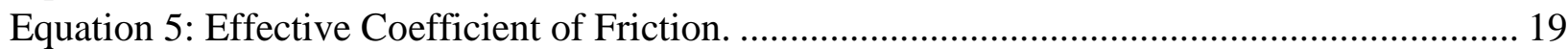

Equation 6: Wrap Angle at the Primary SR $\geq 1$. (Richard G. Budynas) ....................................... 20

Equation 7: Wrap Angle at the Secondary SR $\geq 1$. (Richard G. Budynas) …………….................. 20

Equation 8: Wrap Angle at the Primary SR <1. (Richard G. Budynas) ....................................... 20

Equation 9: Wrap Angle at the Secondary SR <1. (Richard G. Budynas) .................................... 20

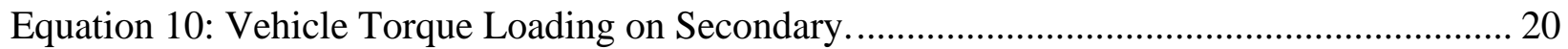

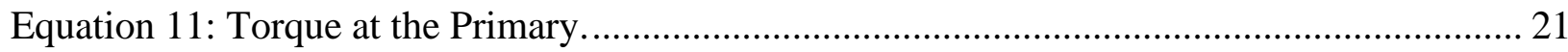

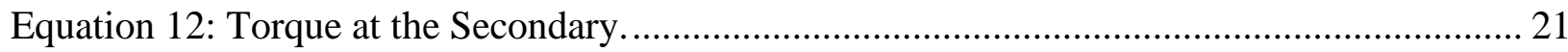

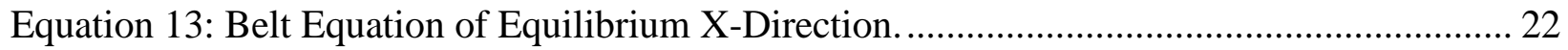

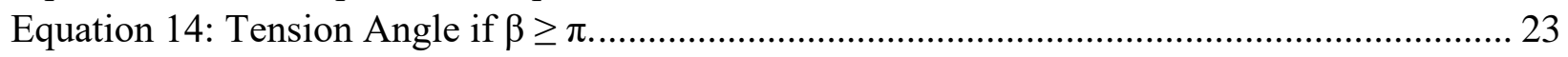

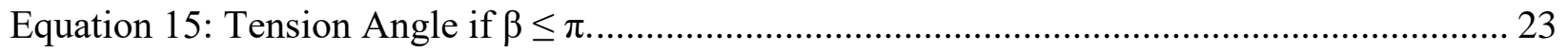

Equation 16: Taut and Slack Side Tension Relationship............................................................. 23

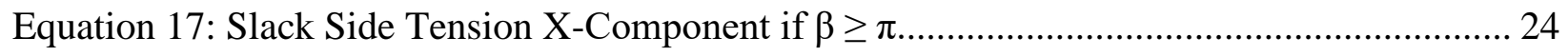

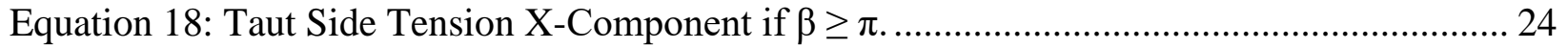

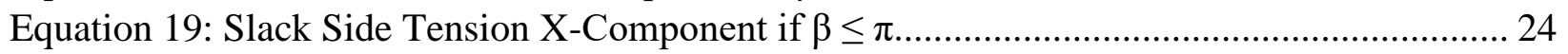

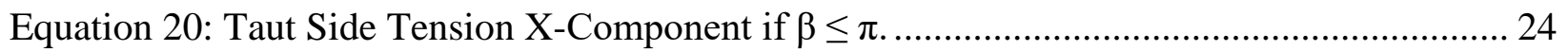

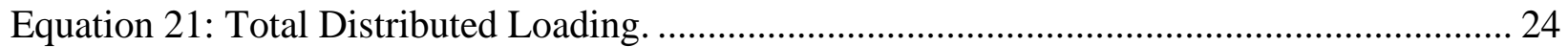

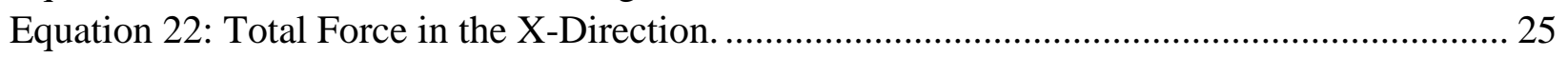

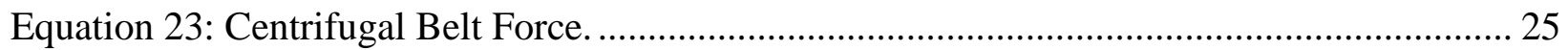

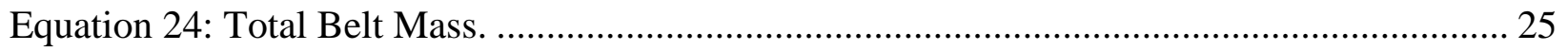

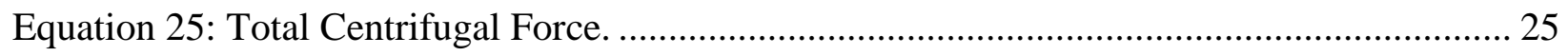

Equation 26: Centrifugal Force Distributed Along the Wrap Angle.......................................... 25

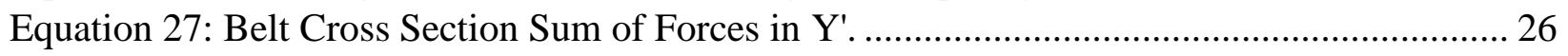

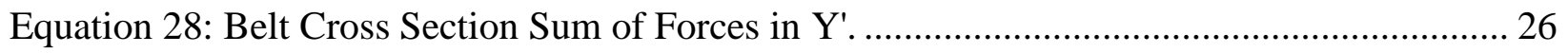

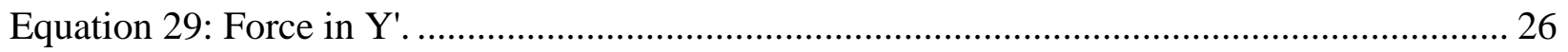

Equation 30: Friction Due to Clamping Force in $Y^{\prime}$................................................................ 27

Equation 31: Friction Due to Radial Force in $Y^{\prime}$...................................................................... 27

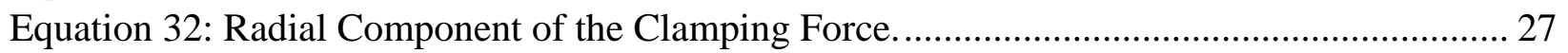

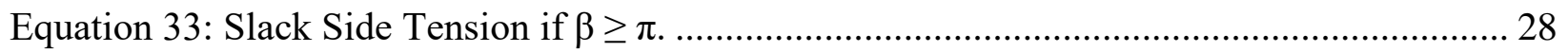

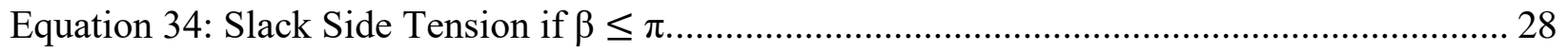

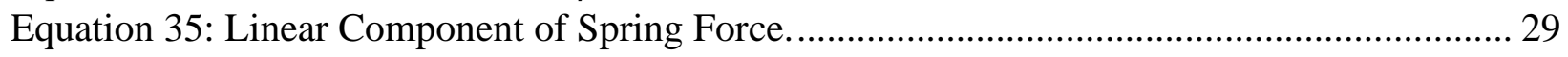

Equation 36: Torsional Component of Spring Force ................................................................ 29

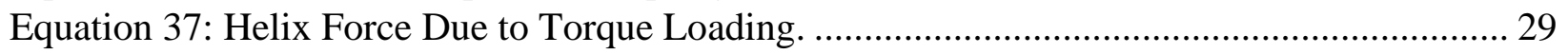

Equation 38: Clamping Force From the Helix. (Aaen)............................................................... 29

Equation 39: Distributed Clamping Force at the Secondary. ........................................................ 30

Equation 40: Maximum Torque at the Secondary..................................................................... 30 
Equation 41: Taut Side Tension at the Primary.................................................................. 31

Equation 42: Slack Side Tension at the Primary. .................................................................... 31

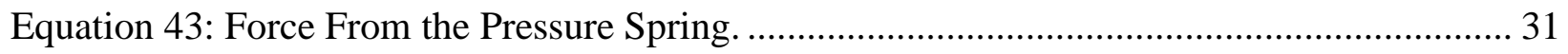

Equation 44: Clamping Force Due to the Flyweights.......................................................... 31

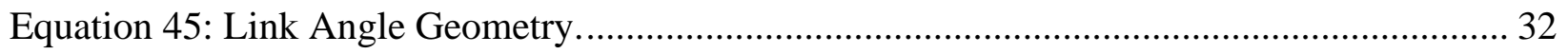

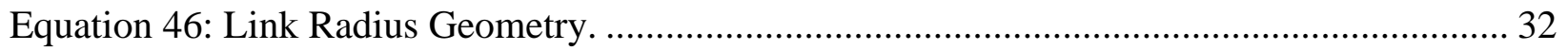

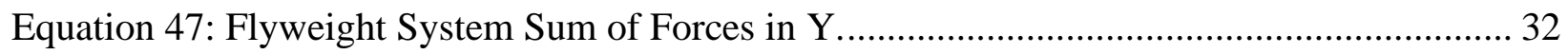

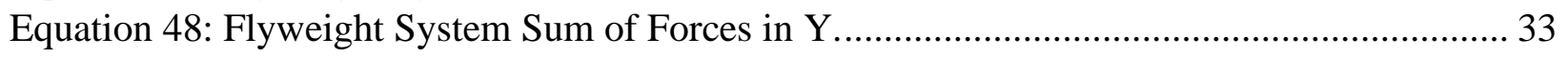

Equation 49: Flyweight System Reaction in the X-Direction. ............................................. 33

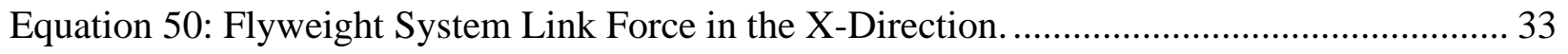

Equation 51: Flyweight System Required Centrifugal Force.............................................. 33

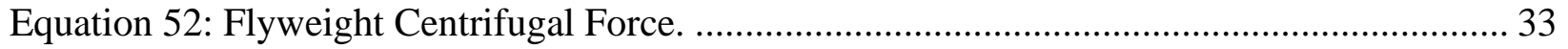

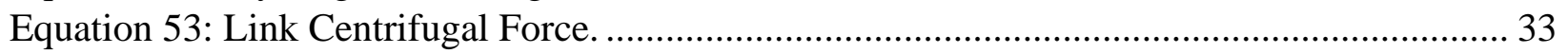

Equation 54: Engine Speed Based on Required Clamping Force ............................................. 34

Equation 55: Maximum Torque Transferrable Primary. (PSU) ................................................ 34

Equation 56: Average Tension Along the Wrap Angle......................................................... 39

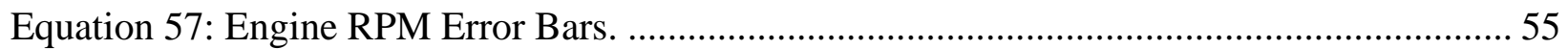

Equation 58: Shift Ratio Based on Angular Velocity....................................................... 55

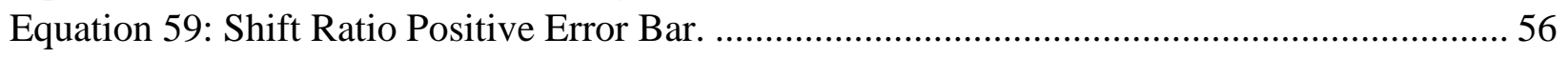

Equation 60: Shift Ratio Negative Error Bar....................................................................... 56

Equation 61: Uncertainty General Formulation.............................................................. 58 


\section{List of Figures:}

Figure 1: A Traditional Gear Box Speed Diagram. (Aaen) ................................................. 3

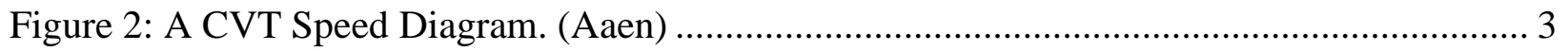

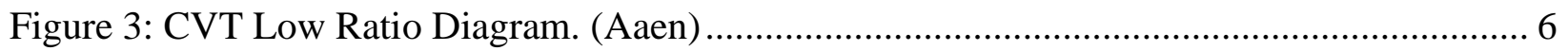

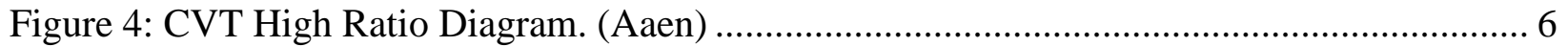

Figure 5: CVT Low Ratio to High Ratio Movement............................................................... 7

Figure 6: Render of a GX9 CVT. (Aztec Baja) ..................................................................... 7

Figure 7: Belt Tension Around a Pulley FBD. (Moradmand, Marcks and Looker) ..................... 8

Figure 8: Wrap Angle Equation Derivation. (Richard G. Budynas)........................................ 9

Figure 9: Sheave Groove Angle Reference Diagram. (Aaen) .............................................. 9

Figure 10: GX9 CVT Torsional Springs. (GE) ................................................................ 9

Figure 11: Helix and Roller Bearings. .............................................................................. 10

Figure 12: Various Helixes With Differing Helix Angles. (Aaen) ......................................... 10

Figure 13: CVT Flyweight System Diagram. (Aulakh)......................................................... 11

Figure 14: Pressure Spring Reference Diagram. (Aaen) ....................................................... 11

Figure 15: CVT Operating Conditions Graph. (Aaen) ...................................................... 12

Figure 16: Custom CVT Spring Test Rig. ...................................................................... 16

Figure 17: CVT Spring Linear Constant Test Setup.............................................................. 16

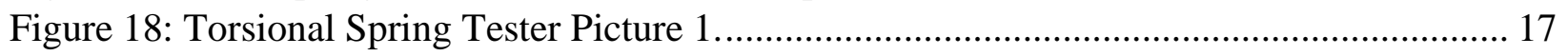

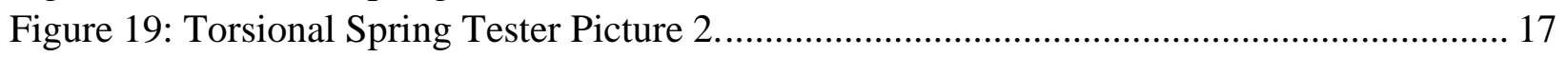

Figure 20: Least Squares Approximation Equation........................................................ 17

Figure 21: Belt Free Body Diagram.................................................................................. 22

Figure 22: Belt Tension Free Body Diagram................................................................. 23

Figure 23: Belt Distributed Loading along wrap angle. ................................................... 24

Figure 24: Belt Cross Section Free Body Diagram. ............................................................ 26

Figure 25: Secondary Helix FBD. (Aaen) ........................................................................... 29

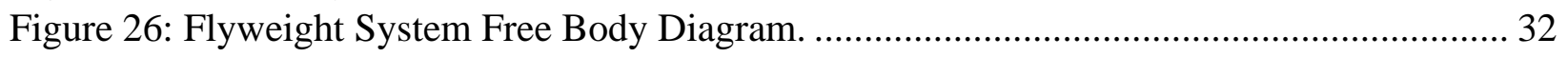

Figure 27: CVT System Assembly Made in SolidWorks..................................................... 35

Figure 28: Primary Sub-Assembly Made in SolidWorks. ...................................................... 36

Figure 29: Secondary Sub-Assembly Made in SolidWorks. ................................................... 36

Figure 30: Belt Model Made in SolidWorks.......................................................................... 37

Figure 31: Belt Model Highlighting Section Division......................................................... 38

Figure 32: CVTech Belt. (Pennslyvania Tech) ................................................................... 39

Figure 33: Enduro Belt. (Gaged Engineering) ................................................................... 39

Figure 34: ANSYS Forces Applied on Belt Model. .............................................................. 39

Figure 35: Land and Sea Water-brake Dynamometer on Test Stand. ...................................... 40

Figure 36: Dyno Table CAD Model. ..................................................................................... 41

Figure 37: Dyno Table with Motor Mounted. ........................................................................... 41

Figure 38: Arduino RPM Monitoring Setup......................................................................... 42

Figure 39: Briggs and Stratton M19 Engine Power Curve.................................................... 44

Figure 40: Briggs and Stratton M19 Engine Torque Curve...................................................... 45

Figure 41: Pressure Spring: Linear Spring Force Vs. Displacement. ...................................... 46

Figure 42: Torsional Spring: Linear Spring Force Vs. Displacement. .................................. 47 


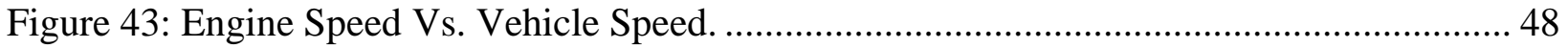

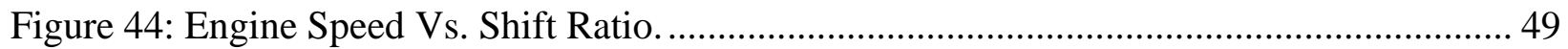

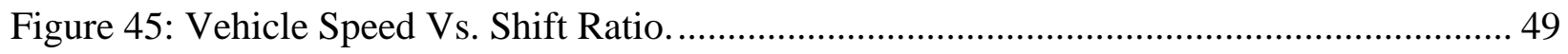

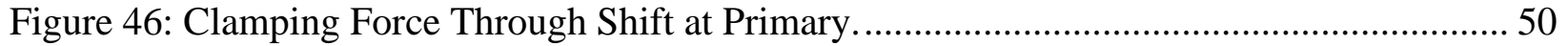

Figure 47: Clamping Force Through Shift at Secondary................................................... 50

Figure 48: Transferrable Torque through shift at Primary................................................... 51

Figure 49: Transferrable Torque through shift at Secondary.............................................. 51

Figure 50: Belt Tension through shift at Primary.............................................................. 52

Figure 51: Belt Tension Through Shift at Secondary........................................................ 52

Figure 52: ANSYS Low Ratio Stress Distribution.......................................................... 54

Figure 53: ANSYS High Ratio Stress Distribution. ....................................................... 54

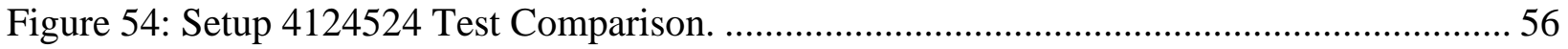

Figure 55: Setup 4223314 Test Comparison. .................................................................... 57 


\section{List of Tables:}

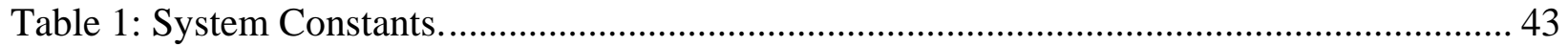

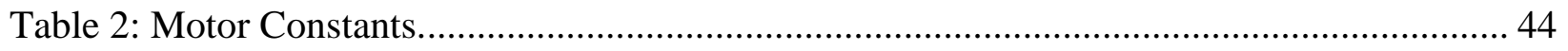

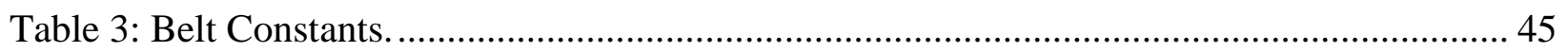

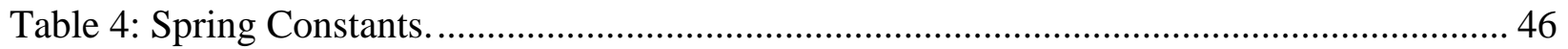

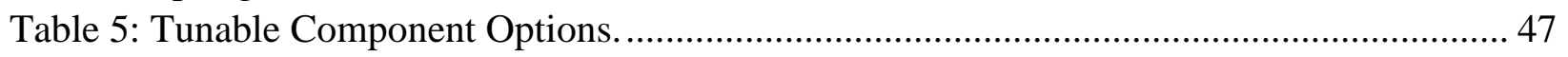

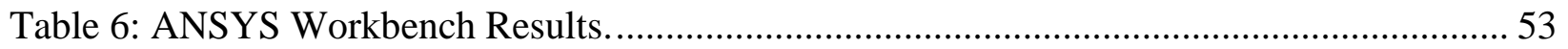

Table of Symbols

\begin{tabular}{|c|c|c|}
\hline Symbol & Units & Description \\
\hline $\mathrm{X}$ & In. & Sheave shift displacement \\
\hline$\lambda$ & Degrees & Secondary angle of twist \\
\hline$\theta$ & Degrees & Primary sheave groove angle \\
\hline$\Phi$ & Degrees & $\begin{array}{c}\text { Secondary sheave groove } \\
\text { angle }\end{array}$ \\
\hline$\gamma$ & Degrees & Hill angle \\
\hline $\mathrm{T}$ & Ft. ${ }^{\text {Ibs }}$ & Torque \\
\hline$\alpha$ & Degrees & Primary wrap angle \\
\hline$\beta$ & Degrees & Secondary wrap angle \\
\hline $\mathrm{T} 1$ & Lbf & Taut side tension \\
\hline $\mathrm{T} 0$ & Lbf & Slack side tension \\
\hline$\mu$ & - & $\begin{array}{c}\text { Nominal coefficient of } \\
\text { friction }\end{array}$ \\
\hline$\mu \mathrm{e}$ & - & $\begin{array}{c}\text { Effective coefficient of } \\
\text { friction }\end{array}$ \\
\hline$\omega$ & RPM & Angular velocity \\
\hline$\delta$ & Degrees & Flyweight ramp angle \\
\hline$\ell$ & In. & Flyweight link length \\
\hline
\end{tabular}




\section{Acknowledgement:}

First and foremost, I would like to thank Dr. Ken Means for your continued guidance and patience through the research process. The experience has been lengthy, and I appreciate the time you have given me. Thank you to Dr. McKenzie for being a point of council throughout the entire process. Thank you to Dr. Musho for lending me your expertise in finite element methods and for giving me guidance in this area.

I wish to recognize my supervisor Jennifer Mandeville for giving me the opportunity to serve as a graduate assistant for MindFit. Without this opportunity I would not have been able to attend graduate school and perform this research.

I would like to thank the 2018-19 and 2019-20 WVU Baja teams for their help in developing the dyno test cell. They were an integral help in designing the dyno table and Arduino DAQ system. Additionally, I would like to extend gratitude to the WVU FSAE ${ }^{\circledR}$ team for playing a huge role in putting together the dynamometer system including plumbing, data acquisition, and monitoring.

The support of my friends has helped me stay the course throughout this process. I would like to specifically thank Dillon Thompson and Dalton Thompson for spending a multitude of hours with me over the past two years. Some days it felt like you cared about my research just as much as I did.

I wish to acknowledge the support of my family: my mother, Susan, my father, Dennis, and my siblings, Tara and Justin. Thank you for always offering words of encouragement. There were many times the mountain felt insurmountable, but I knew I could come to you for guidance.

Finally, I would like to thank Rebekah Rhodes for your immense support. You have always been a shoulder to lean on and an ear to listen. I would not have been able to accomplish this without you. 


\section{Introduction:}

Continuously variable transmissions (CVT) is about as wide sweeping of a term as "engine". Today there are a multitude of CVT systems which vary based on their application. It is said that they were first conceptualized by Leonardo Da Vinci in the $15^{\text {th }}$ century. Since then they have grown rapidly in popularity, stretching into a variety of fields. This section will describe a brief history of the CVT, the many types that exist today, and how they became so popular.

\section{CVT History:}

One of the first CVTs patented and produced commercially was the Variomatic, built by Hub van Doorne in the late 1950s. He was the co-founder of Van Doorne's Automobiel Fabriek (DAF), who sought out to produce a small, affordable car, with an automatic transmission. They succeeded by implementing this system in the DAF 600 vehicle, which was instrumental to the popularization of CVT systems in automobiles. The Variomatic was a simple mechanical design with a rubber V-belt and pulley system. The DAF passenger car division was later sold to Volvo, along with the patent to the Variomatic (Holloway and Buckley).

Volvo continued to implement CVTs in their consumer vehicles until the 1990s. Other car manufacturers, such as Subaru, Honda, Ford, and Nissan took interest in the technology as well. Finally, the first production automobile with a CVT was offered in the United States in 1989, the Subaru Justy. Today the technology has advanced in several directions including application, system design, and belt type.

\section{Metal Push Belts:}

In some applications with higher power production the use of a rubber belt isn't suitable. For these cases use of a metal push belt can be a better choice. These belts are constructed from hundreds of individual metal rings strung together. These belts won't deform as easily as rubber belts, making them more consistent under high loading conditions. The disadvantages to consider is friction between the belt elements. As the CVT shifts, the individual rings will flex in compression, which can cause losses between each other.

\section{Hydrostatic CVT:}

Hydrostatic CVTs offer many benefits of a mechanical CVT with fewer parts and reduced friction. They work by use of variable displacement hydraulic motors, which convert rotational energy into fluid flow. Performance can be altered by adjusting the motors, rather than swapping components. These systems work at high pressure, requiring robust components. This increases response time to changes in loading condition, such as back shifting after hitting a hill. Overall, this CVT works best in consistent, low speed conditions, making it a great choice for heavy machinery, such as tractors (Johnson). 


\section{Electric CVT:}

CVTs have become more popular in commercial vehicles for their efficiency benefits. However, these CVTs are almost always electric CVTs. This is because it is significantly easier to control performance when using electronically actuated sheave displacement. With this type of CVT, sensors are used to monitor engine RPM, torque loading on the vehicle, and other external parameters such as hill incline. These sensors can then adjust sheave displacement and CVT performance in real time. These CVTs hold a great advantage over mechanically controlled CVTs because of this ability to change control scheme during operation.

\section{Mechanical CVT:}

Mechanically controlled CVTs operate with counteracting forces. These forces work together to control performance throughout operation. The primary benefit of this type of system is that they operate on their own. They don't require electric power of any kind. The primary downside of this control method is that changing the control scheme requires physically changing components. Optimizing a setup like this requires predicting the conditions the system will endure and making choices accordingly. Furthermore, the setup options are limited by available choices. Sometimes manufacturing of custom components is required.

\section{Snowmobiles:}

Many advancements in rubber belt CVTs have developed in the snowmobile industry. These machines encounter a variety of loading conditions such as tight cornering, quick inclines/declines, and thick patches of powder. Snowmobiles rely heavily on momentum to keep from getting stuck in such low friction terrain. This means the powertrain must react quickly to stay in an efficient operating range. In its early days, riders quickly realized traditional gear boxes would simply not work for these conditions because they would have to cut power to change gear ratios. Interrupting power output to change ratio was simply not an option to take on the shifting loads. CVTs offer the ability to change ratio without dropping off the power peak. Figure 1 And Figure 2 show the differences between these two systems. If these diagrams were for an engine with a power peak around 9000 RPM, the CVT allows the system to stay on the power peak while increasing speed. Whereas the traditional gear box must reduce RPM to shift into the next gearing ratio. 


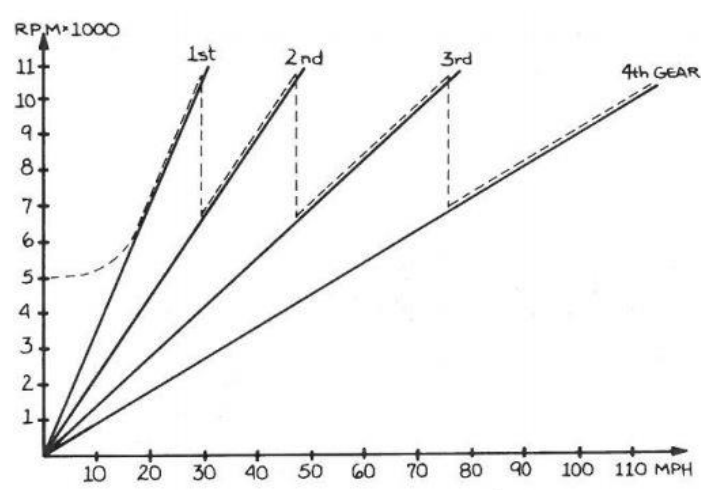

Figure 1: A Traditional Gear Box Speed Diagram. (Aaen)

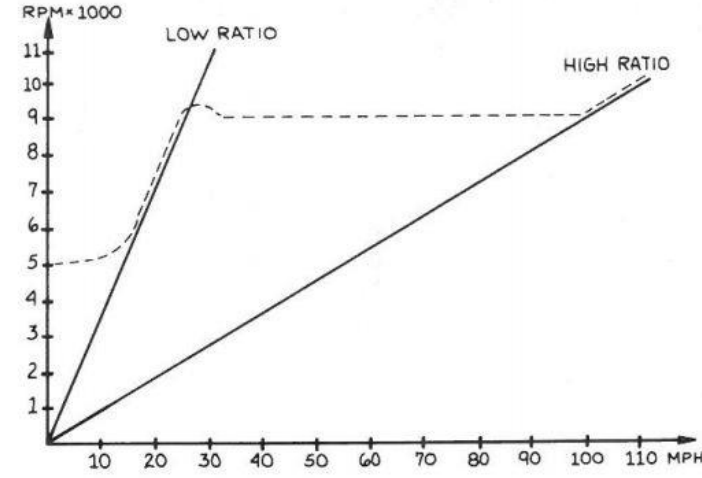

Figure 2: A CVT Speed Diagram. (Aaen)

Designers knew the potential benefits of CVTs and worked to make these dreams a reality. With lower horsepower machines the systems worked well, reacting to varying loads and adjusting ratio accordingly. Problems started to arise as riders wanted to use higher horsepower engines. Early CVT design didn't handle clamping force very efficiently and couldn't react quickly enough to high loading conditions. These factors subjected the belt to loads much higher than required, reducing overall life drastically. This led to the development of torque feedback ramps and more efficient flyweight mechanisms. The torque feedback system is critical for applications with varying loads. The benefits even stem to low horsepower machines where using horsepower efficiently is even more important.

\section{Baja SAE ${ }^{\circledR}$ Application:}

Baja $\mathrm{SAE}^{\circledR}$ teams face many of the same challenges as snowmobilers. The variety of dynamics events test vehicles in sled pulls, acceleration runs, hill climbs, stability, and suspension courses. To be competitive, the powertrain must be able to adjust to these conditions quickly and operate efficiently. The final event, the endurance race, puts the difficulties from all these events together, requiring a system that can adjust accordingly on its own. All teams are given the same Briggs and Stratton $10 \mathrm{Hp}$ engine, so powertrain design is based on how this power is used.

The strengths of a CVT system directly align with the challenges in these events. The system can adjust quickly to varying load conditions from obstacle to obstacle. Most importantly, using a CVT ensures teams are getting the most out of the $10 \mathrm{Hp}$ they are provided. Part of the power train design lies in selecting a proper gearing ratio between the CVT output and the wheels. But the competitive edge really comes from a CVT that reacts quickly and efficiently to changing loads.

\section{Importance of Tuning}

The fact that CVT systems are so flexible means that they can't simply be bolted onto any machine. The efficacy of a CVT is only as powerful as the tuning process used to apply it to that system. A system that isn't tuned properly will affect efficiency, reaction speed, and could even prevent the system from shifting into high gear. 
Traditionally tuning is performed by gathering test data and analyzing the effects of changing components. This method requires an immense amount of data and time. Tuning this way can take weeks or even months of time and hard work. Alterations to any other component of the vehicle such as suspension setup, tire diameter, or even weight, greatly reduces usefulness of past data. This thesis lays out the groundwork for a computational approach. This method involves tuning based on understanding system components, modeling the system, and empirical analysis. 


\section{Literature Review:}

The primary disadvantage of using a mechanically controlled CVT is the inability to change the control scheme during operation. Adjusting the control scheme requires changing physical parts, which requires time and tools. This makes it particularly beneficially to develop a model to predict CVT performance without physical testing. To circumvent this problem, many schools such as UCLA and Virginia PolyTech University use electric CVTs. These systems allow on the fly adjustments to control schemes and observe changing conditions in real time. The primary downside to these CVTs is they require significant research and development to create a reliable system. The methods outlined in this thesis are designed for useful for the current WVU Baja team. This implies that they must be applicable to their current selection of CVTs which are both mechanically controlled systems.

Jot Aulakh, with the National Institute of Technology, published a journal article detailing his approach to developing a genetic algorithm-based approach for tuning (Aulakh). His research was conducted regarding a mechanically controlled CVT. The algorithm works by firstly identifying a controlling fitness equation. This equation is used to observe all possibilities for force balance and compares them to specified criteria. Secondly, the user must specify a set of criteria that must be met at each "step". The algorithm then scores each possibility based on its deviance from the originally specified criteria. Once all values meet the criteria, the algorithm then steps and repeats. Using this method develops the optimal force balance through operation to meet the specified criteria.

There are a few disadvantages to Aulakh's approach. Primarily this method finds the absolute optimal force balance but doesn't consider any physical limitations. In other words, the algorithm may specify a required force of precisely $45.6 \mathrm{lbs}$. but doesn't consider what is possible with available parts. This implies that some form of electronic actuation where precise forces can be applied at any instance is required. Secondly, the fitness equation in this paper doesn't consider torque input from the motor or exterior conditions such as loading on the vehicle. Finally, the fitness equation doesn't consider centrifugal forces of the belt as it wraps around the CVT.

The methods outlined in this thesis finds the best possibility based on a set of pre-defined options. These options are defined by the collection of physical parts available for the CVT. Finally, the governing equations derived in this paper consider torque input, external loadings, and the centrifugal forces of the belt. These factors all greatly impact vehicle performance in real world conditions; creating a more accurate model. 


\section{System Components and Variables:}

Companies such as Polaris, Jatco, and Arctic Cat manufacturer CVTs with similar core designs. The differences lie in the application they are intended for. Polaris and Arctic Cat primarily create CVTs for use in their snowmobiles. On the other hand, Jatco designs theirs with commercial automobiles in mind. These differences lead to critical changes in design, which change the way the system responds. Understanding the design choices made is critical for proper use and performance of the CVT.

The CVT currently used by the WVU Baja team is the GX9, created by Gaged Engineering (GE), based out of Arizona. GE has been designing CVT systems since 1998. The GX9 was created specifically for use with the Briggs and Stratton M19 engine, the motor used by all Baja teams. Calculations and testing approaches are applicable to any CVT, but they were performed regarding this system.

\section{Sub Systems:}

The GX9 consists of three major sub systems: the primary (driver), the secondary (driven), and the belt. The primary and secondary vary in their components, but in general they are both considered pulleys. When discussing factors that generally apply to either the primary or secondary, they may be referred to as pulleys. Both pulleys have angled sheaves constructed of aluminum. The angle between sheaves varies between CVTs and is referred to as the sheave groove angle. One of the sheaves is moveable, while the other is fixed in place. The moveable sheaves squeeze against the belt creating clamping forces. The balance between these clamping forces control the way power is transferred, and the shift characteristics. While shifting, the primary sheaves close on the belt, pushing it further out on its sheaves. Meanwhile, the secondary sheaves are forced open, and the belt is pulled in lower on its sheaves. This dynamic is shown in Figure 3 and Figure 4 with the primary on the left and the secondary on the right. Figure 5 shows sheave movement while shifting. The left image represents low ratio, where the primary radius is smallest, and the secondary radius is larger. The right image represents high ratio where the sheaves at the primary have shifted closed, increasing the radius. At this point the sheaves at the secondary have been forced further apart decreasing the radius.

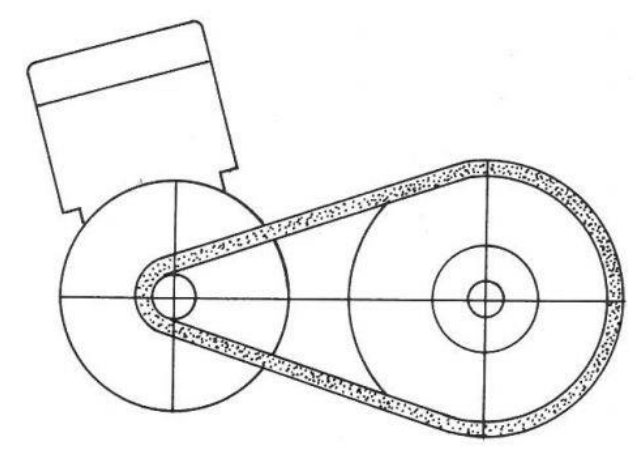

Figure 3: CVT Low Ratio Diagram. (Aaen)

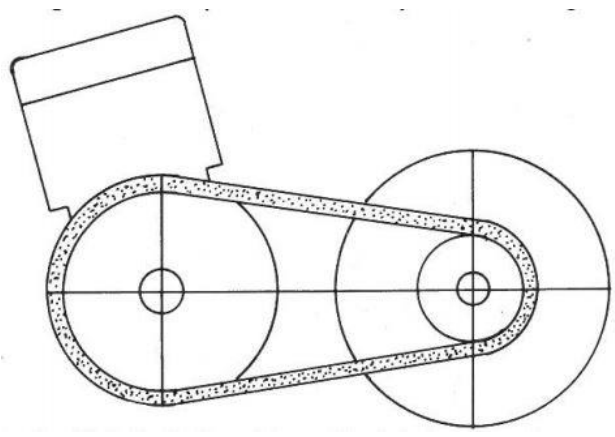

Figure 4: CVT High Ratio Diagram. (Aaen) 

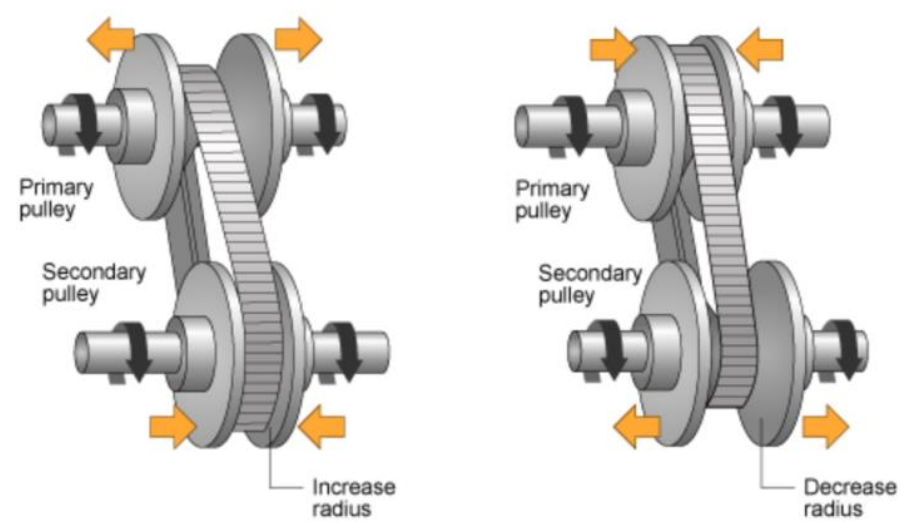

Figure 5: CVT Low Ratio to High Ratio Movement.

The primary is fixed on the engine output shaft. It contains a linear pressure spring and flyweight system sliding along steel ramps. The secondary is connected to the gearbox input shaft. It creates clamping force through torque feedback and a torsional spring. These forces work against an angled helix, which transfers this torque into clamping force. The belt is a cogged rubber Vbelt with stiff chording. The components of these sub systems work against each other to achieve desirable outputs. Figure 6 gives a view at the components within each subsystem. The secondary is on the left highlighting the helix and torsional spring. The helix is on top with an angled slot and cams riding along it. The torsional spring is held between this helix and the fixed sheave. The primary is on the right, highlighting the flyweight system with ramps and the weights that work against them. The pressure spring is within the metal housing in the center of the flyweight system. More detailed explanations of precisely how each of these components work will follow in each subsystems section.

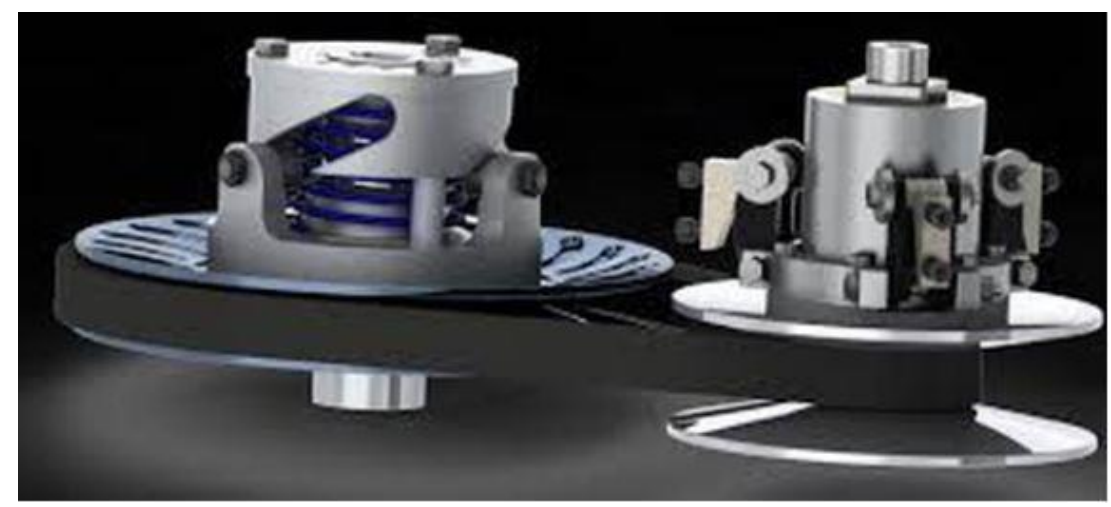

Figure 6: Render of a GX9 CVT. (Aztec Baja)

The Belt:

In a CVT, the belt transfers power from the primary to the secondary through tension. The GX9 uses a cogged rubber V-belt. The cogs allow the belt to be flexible and bend around a tight radius. The chording along the belt makes the belt stiffer in tension, making it more difficult for the belt to deform under loading. Stretching of the belt would cause tension loss and reduce the efficiency of power transfer. The $\mathrm{V}$ shaped cross section allows the belt to sit properly in the sheave groove and reduces slipping under load. 
While the belt is pulled from the primary to the secondary it is loaded in tension. One end of the belt will always have a higher tension than the other side. The end with higher tension is known as the taut side, while the other is the slack side. This difference in tension transfers power produced by the engine. In a perfectly efficient system, power is constant between these two sub systems, but the torque and angular velocity varies. This is due to the shift ratio, which is the ratio of the secondary pitch radius to the primary pitch radius. The shift ratio gets smaller as the system shifts. While the shift ratio is greater than one, torque and angular velocity are increased from the primary to the secondary. Figure 7 details this relationship with $T_{2}$ representing the taut tension and $T_{1}$ the slack tension.

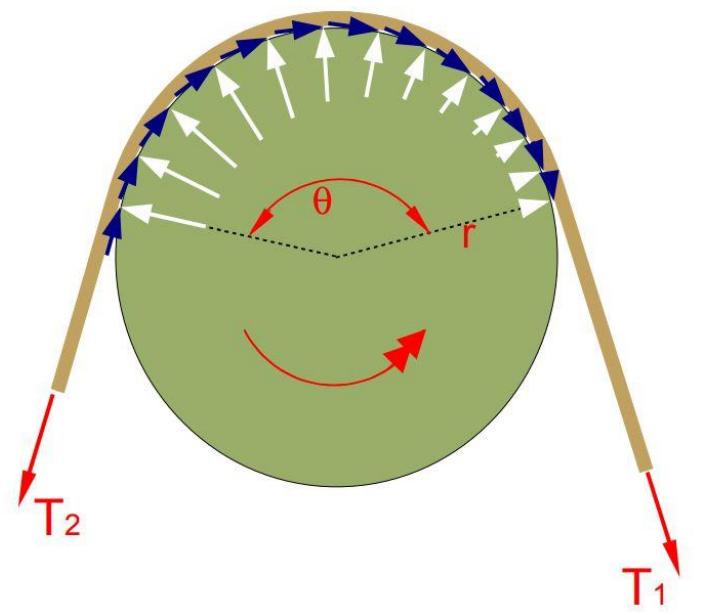

Figure 7: Belt Tension Around a Pulley FBD. (Moradmand, Marcks and Looker)

There are three variables of interest when considering the belt. They are the sheave angle, the angle of wrap, and the belt centrifugal force. The sheave angle, measured in radians, is half of the sheave groove angle (Figure 9). This value increases the effective coefficient of friction between the belt and sheave surface. Steeper sheaves create a higher effective coefficient of friction. The angle of wrap, measured in radians, is the length of belt in contact with the sheave surface (Figure 8). A larger pitch radius correlates to a larger wrap angle. These two values directly control the relationship between the slack and taut side tension. Higher wrap and sheave angles will increase the difference between these two tensions, giving a higher taut side tension. Finally, the belt centrifugal force will uniformly affect the tension around the sheave. Figure 8 highlights the distribution of force as the belt wraps around the pulley. A higher centrifugal force will increase the tension on both sides of the pulley. This is an important consideration when analyzing the force balance between the primary and secondary. 


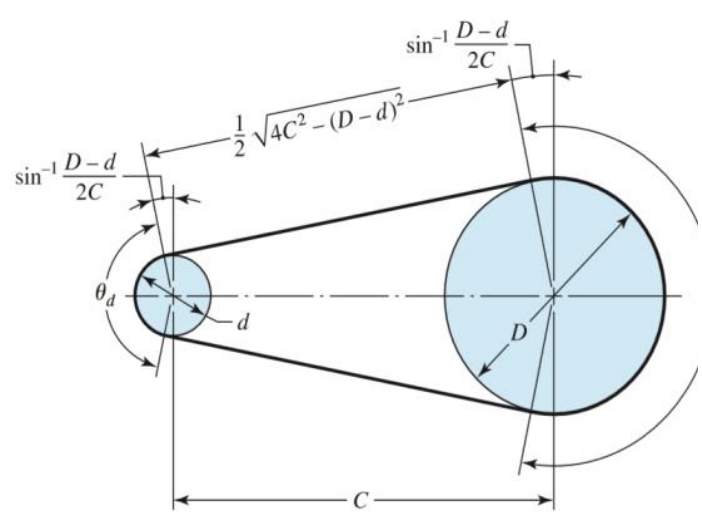

Figure 8: Wrap Angle Equation Derivation. (Richard G. Budynas)

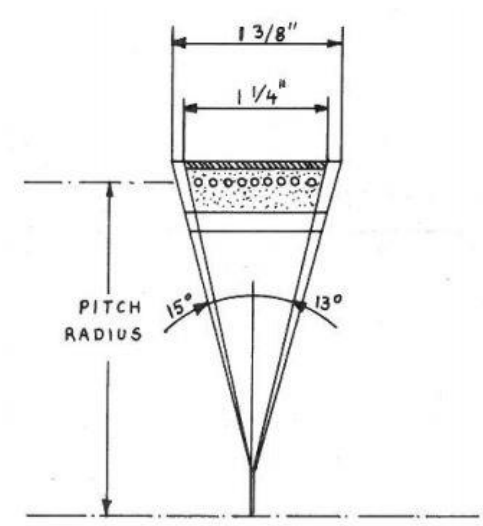

Figure 9: Sheave Groove Angle Reference Diagram. (Aaen)

\section{Secondary:}

The secondary is responsible for controlling efficient power transfer into the gear box. Power loss occurs if the clamping force isn't optimal. If it is too low, the sheaves won't be able to hold onto the belt and slipping will occur. If the clamping force is too high there will be excess friction and tension in the belt. This will decrease the efficiency of the system and increase wear on the belt.

The secondary clamping force is the summation of three forces, two of which come from the torsional spring, and the last from torque feedback. The torsional spring, shown in Figure 10, is designed as a normal helical torsion spring, which resists twisting. However, it also has space between its wraps and is compressed as the secondary sheaves turn and pull apart. This means the spring not only resists twisting, but it also resists compression. For this analysis, the torsional spring forces are broken into a torsional force and a linear force. Two separate spring constants are used, and the associated forces are observed separately. The linear force works directly into the sheave creating clamping force. The torsional force creates torque on the helix, where a portion of it produces clamping.

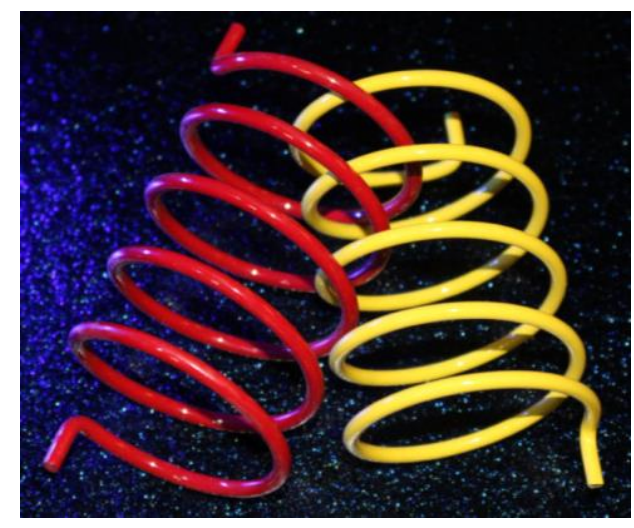

Figure 10: GX9 CVT Torsional Springs. (GE) 
The other force working on the helix is the torque feedback. Torque experienced at the secondary, in addition to the torsional spring, push roller bearings into the helix. This force produces clamping force and rides up the helix during shift. In low ratio, torque is high, thus the clamping force from feedback is high. As the CVT shifts out, the shift ratio decreases, lowering torque at the secondary. Less clamping force is required in high ratio because there is less torque to transfer. Ideally, while torque is high the clamping force is high, and as the CVT shifts out, torque decreases and clamping force from feedback decreases.

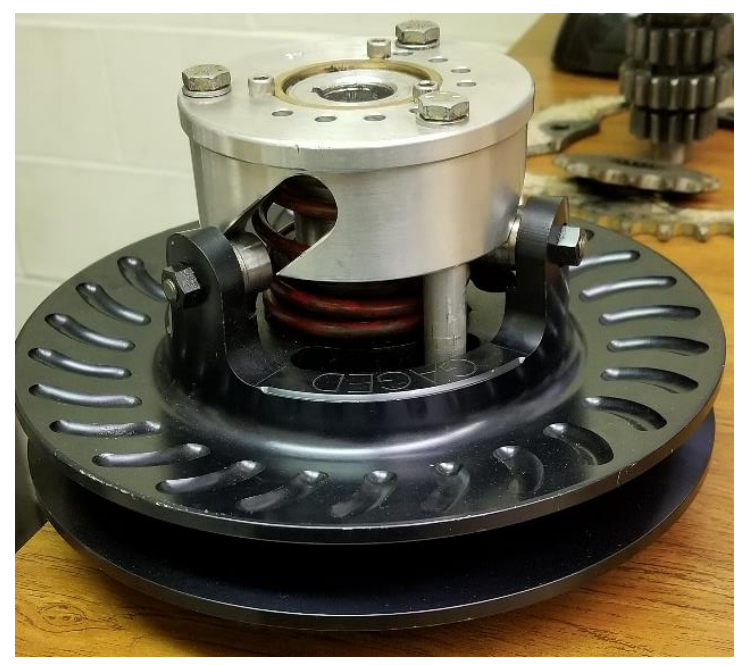

Figure 11: Helix and Roller Bearings.

There are several variables to consider when tuning on the secondary. The torsional spring has an installed compression and twist. These define the linear and torsional pretensions. As the CVT shifts the sheaves are pulled apart twisting and compressing the torsional spring. The linear and torsional rates must be considered to predict the associated forces produced while shifting. Finally, the choice of helix, and associated helix angle must be considered. This angle determines the percentage of torsional forces that are converted into clamping force. Examples of helixes are shown below in Figure 12. The GX9 has several helixes to choose from with varying profiles. Most have a linear profile with a consistent angle. There are also progressive helixes where the angle changes as the CVT shifts for more precise tuning options (Gaged Engineering).

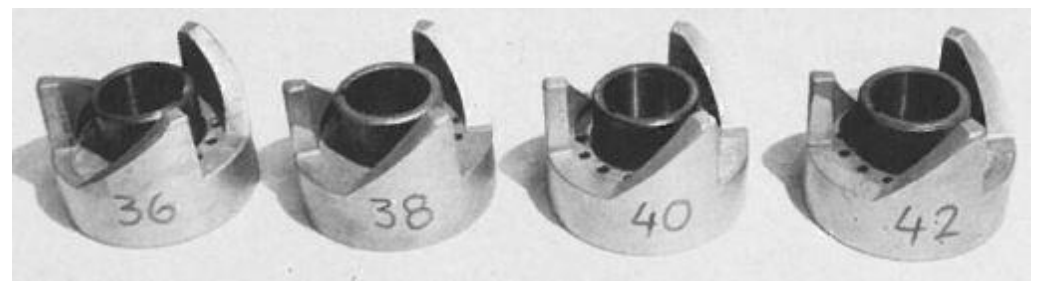

Figure 12: Various Helixes With Differing Helix Angles. (Aaen)

\section{Primary:}

The primary is used to control engine speed. Before tuning, the desired engine speeds at specific operation points should be determined. These points of interest depend on where the engine is producing max power and where it produces max torque. The engine speed is directly 
controlled by the clamping force at the primary. Adjusting this force is the best way to tune the primary without affecting performance of the secondary.

The same clamping force can be produced at different engine speeds by adjusting the relationship between the pressure spring and flyweight system. The pressure spring resists the sheaves from shifting while the flyweights work to push the sheaves closed. The difference between these is the resultant clamping force on the belt.

As the engine turns, the flyweights create centrifugal force which work against ramps in the housing. The magnitude of this force is dependent on the working radius, engine speed, and flyweight mass. Increasing the total mass of the flyweights would increase the force produced at the same engine speed. The ramp angle determines the percentage of this force effecting the moveable sheave. This dynamic is detailed in Figure 13. A steeper angle will produce more clamping force. As the CVT shifts, the flyweights slide up the ramps, and increase the working radius, which in turn increases the centrifugal force produced at that speed.

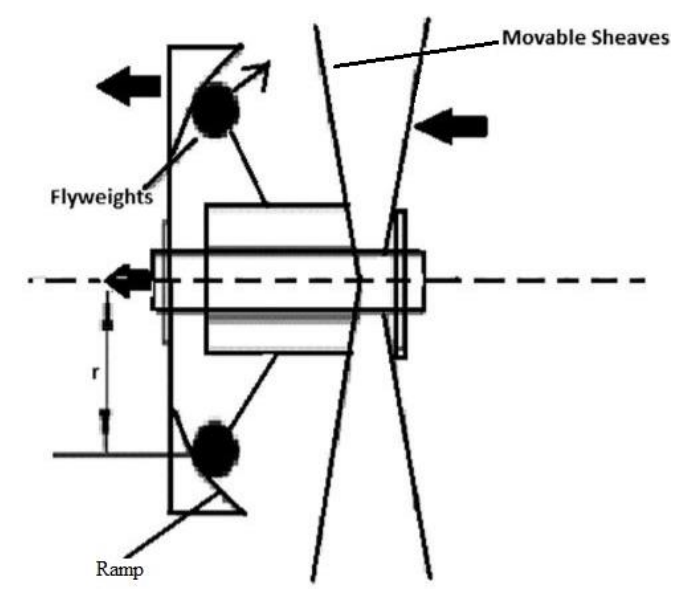

Figure 13: CVT Flyweight System Diagram. (Aulakh)

The pressure spring, shown in Figure 14, is a straight linear helical spring. This means that as the spring is compressed the force produced increases following a linear relationship, known as the spring constant. Knowing this value and the installed length of the pressure spring gives the pretension in the primary. As the sheaves are forced closed by the flyweights the spring will continue to compress, further, resisting the flyweight system. The balance between the flyweight system and pressure spring controls the engine speed throughout operation.

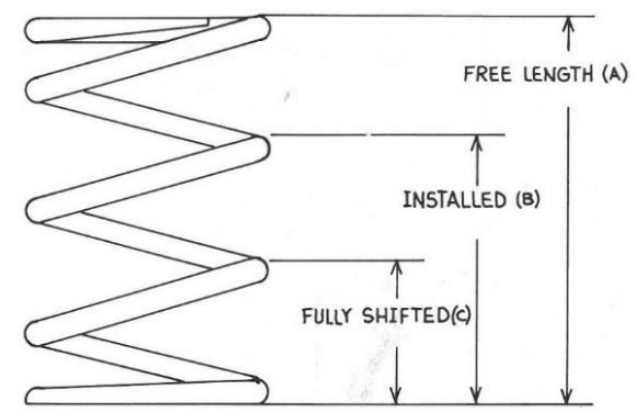

Figure 14: Pressure Spring Reference Diagram. (Aaen) 


\section{Operating Conditions:}

To properly tune a CVT it is important to understand how it will respond during use. The system goes through many phases as the machine accelerates to high speed. These phases, and additional points of interest, are shown below in Figure 15. (A) represents engine speeds below idle, so the system should never be in this range. Point (B) is the engine idle speed, where the vehicle should remain stationary. $(\mathrm{C})$ is the clutching phase, where engine speed is just building up and the vehicle is just starting to move. (D) represents low ratio acceleration. In this range the belt has been fully engaged and is transferring power. Point (E) represents the shift point, where the sheaves are just starting to move. (F) is the shifting phase, where the sheaves are moving, and shift ratio is changing. Finally, $(\mathrm{G})$ represents high ratio acceleration, where the sheaves have fully shifted into high ratio.

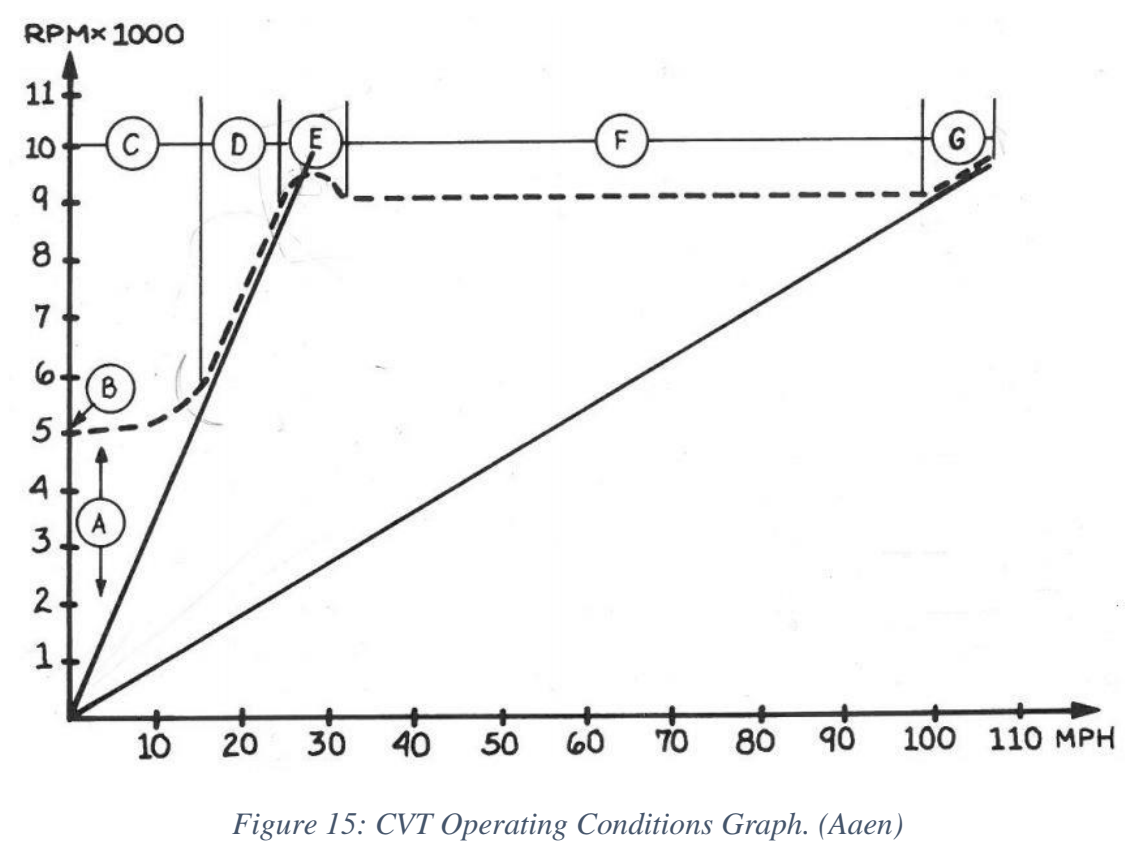

\section{Clutching Phase:}

While the engine is idling, the flyweights shouldn't be producing enough force to overcome the pretension of the pressure spring. This allows the sheaves to slip freely past the belt, keeping the vehicle stationary. The idle speed of the engine should be set below the engagement speed. As the engine rpms increase, the flyweights will eventually overcome the pretension in the pressure spring pressing the primary sheave into the belt. The clamping force builds up and the sheaves start to engage the belt. This is known as clutching. During this phase there is excessive slip on the belt and the vehicle begins to move forward.

\section{Low Ratio Acceleration:}

The next phase begins once the flyweights have built up enough clamping force to fully engage the belt. At this point the belt is no longer slipping, and power is being fully transferred from the primary to the secondary. After engagement the vehicle will accelerate in low ratio. The 
belt is sitting in the bottom of the primary and the top of the secondary. The sheaves remain stationary because the flyweights have overcome the pretension of the pressure spring but haven't created more clamping force than the secondary.

\section{Shift Out:}

The system will continue accelerating in low ratio until the taut side tension is higher at the primary than the secondary. This is known as the shift out point, when the primary sheaves can start closing. As they close, the belt is pushed further out on the primary and is pulled into the secondary, forcing its sheaves apart.

While shifting, the balance of taut side tension between the primary and secondary changes continuously. The primary sheaves closing compresses the pressure spring increasing spring force. It's also increasing the working radius of the flyweights, increasing centrifugal force. On the secondary the sheaves open. This will compress and twists the torsional spring, increasing spring force. This movement also decreasing the shift ratio, lowing the clamping force from torque feedback. Balancing these forces is complex and depends on a variety of variables. Controlling the shift requires consideration of all points along the shift, and the continually changing force balance. At all points the engine RPM will increase to a point where the primary tension just overcomes the secondary tension, the sheaves will move just a bit more, and the force balance will need to balance once again.

\section{High Ratio Acceleration:}

When the CVT reaches full shift, the sheaves will no longer move. All spring forces are now at their maximum value. The shift ratio will remain constant, meaning the torque will only vary slightly based on the torque produced by the engine. These factors mean that the taut side tension at the secondary will remain effectively constant. The engine will now be able to rev higher, pushing the primary tension further past the secondary tension. The vehicle will continue accelerating along in high ratio, with increasing engine speed. 


\section{Tuning Objectives:}

A CVT is so versatile because of its vast number of setup combinations. Each variable component has several options that can be swapped in with different spring constants, masses, ramp angles, etc. This allows the tuner to precisely dial in the performance to meet their performance objectives. The primary goal for the Baja team is to have the CVT stay on the power curve during shift out. Secondary objectives include controlling the engagement speed and increasing the efficiency of power transfer through the secondary.

\section{Straight Shift:}

During operation the CVT will primarily reside somewhere in the shift range. The objective during shift out is to have the belt move from low to high ratio while keeping the engine operating on the power peak. Knowing this defines a desired engine speed while shifting. Throughout the shift the primary tension must just overcome the secondary tension at this desired speed. If this is done successfully the engine speed will stay nearly constant, at the power peak, along the entire shift ratio. Staying on this peak will ensure the engine is putting as much power to the wheels as possible.

\section{Engagement Speed:}

Engagement marks where the belt is first fully engaged, and the vehicle is just taking off. For optimal performance the system should be tuned to have full engagement occur where the engine is creating peak torque. This will provide better take off climbing hills from a stop, and faster acceleration off the line.

\section{Efficient Power Transfer:}

Efficient power transfer along the belt is primarily controlled by the secondary. The objective when tuning the secondary is to produce just enough clamping force to prevent slipping. The general rule of thumb for dynamic machines is to shoot for no more than $20-30 \%$ of the clamping force required (Aaen). This will prevent slip during high loading scenarios such as jumps. Using this rule creates an optimal operating window with a minimum and maximum clamping force at the secondary. 


\section{Methods: Empirical Analysis and Testing}

A combination of calculation and testing were performed to predict performance of the CVT in different setups. First, data was gathered using a spring testing rig to calculate constants of the various springs. Second, a variety of equations were used in MATLAB to determine the force balance throughout the CVT during operation. A design envelope was determined based on the tuning objectives and optimal setup combinations were noted if the CVT remained in this envelope. An FEA model was created in ANSYS to simulate various loading conditions and confirm forces calculated empirically. Finally, the CVT was tested on a water brake dynamometer. This testing gathered speed and torque data to compare the actual performance with the expected performance found through calculation.

\section{Spring Constants:}

A critical aspect of controlling CVT performance is understanding the spring forces. Pretension and shift forces produced by the pressure and torsional springs are dependent on their associated spring constants. A total of 9 springs were used for testing, 5 pressure and 4 torsional. All springs used were provided by GE and varying constants are denoted by their color. In addition, some springs were from a previous Baja team to see how wear and age would affect their performance. These springs were approximately 3 years old and used at 2 competitions. Pressure springs used were new black, orange, and purple springs in addition to one old black spring. Torsional springs tested were new red and yellow springs as well as old red and yellow springs.

In many cases the manufacturer will list their spring constants online. GE does not have any data for their springs presented, so these values required testing in house. Testing methods were created based on Hooke's Law, which has two forms, one for linear springs (Equation 1) and one for torsional springs (Equation 2). To calculate the various spring constants for the CVT these equations were rearranged and solved. For linear constants testing must be able to determine linear displacement and the associated force response. For torsional constants testing must be able to determine angular displacement and the associated torque response.

$$
\begin{gathered}
F_{\text {Linear }}=\kappa_{\text {Linear }} * X \\
\text { Equation 1: Hooke's Law Linear Formulation. } \\
F_{\text {Torsional }}=\kappa_{\text {Torsional }} * \lambda \\
\text { Equation 2: Hooke's Law Torsional Formulation. }
\end{gathered}
$$

\section{Spring Testing Rig:}

A testing jig was designed to be able to test both linear and torsional constants with minimal setup changes. First, the varying diameters between the pressure spring and torsional spring needed to be considered. A cylinder was made for each spring just larger than its diameter to form the walls. This allows the spring to be compressed or twisted without bucking over. The base of the test rig has a quarter inch step to hold the cylinder in place. The SolidWorks design for this setup is shown below in Figure 16. 


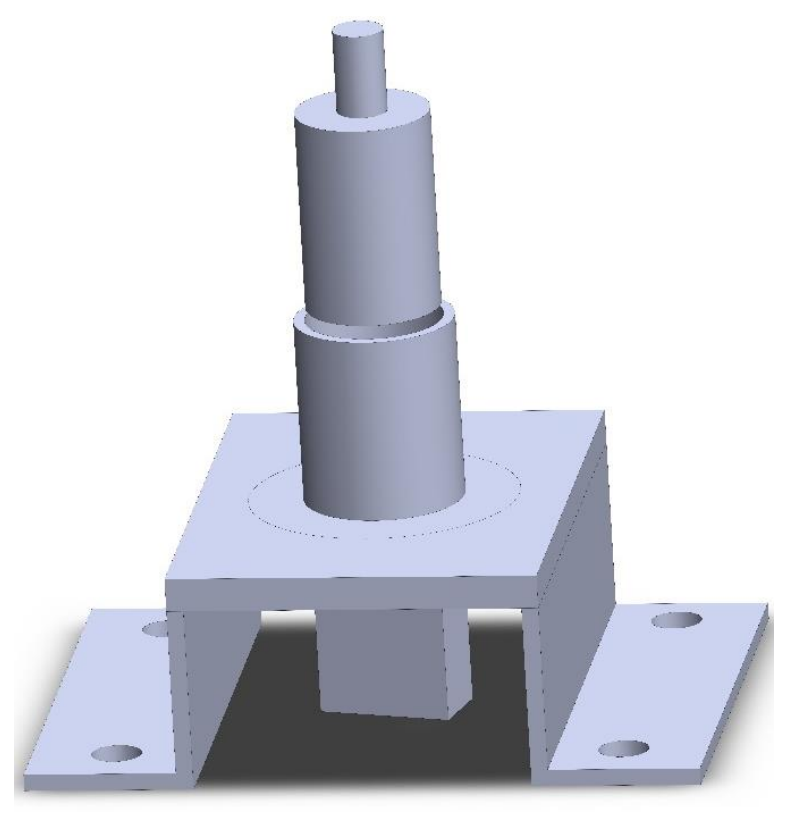

Figure 16: Custom CVT Spring Test Rig.

For linear tests, spacers were made to sit inside of the cylinder and support the bottom of the spring. This spacer is bolted onto the top of a load cell, which monitors the force applied to it. To measure displacement in small increments the head of a mill was used. A top spacer was made with a stem that can fit into a standard mill collet. This spacer was made to slide inside of the cylinder, so that it can fully compress the spring. Finally, holes were drilled into the base of the rig to secure to the mill bed with T-Nuts.

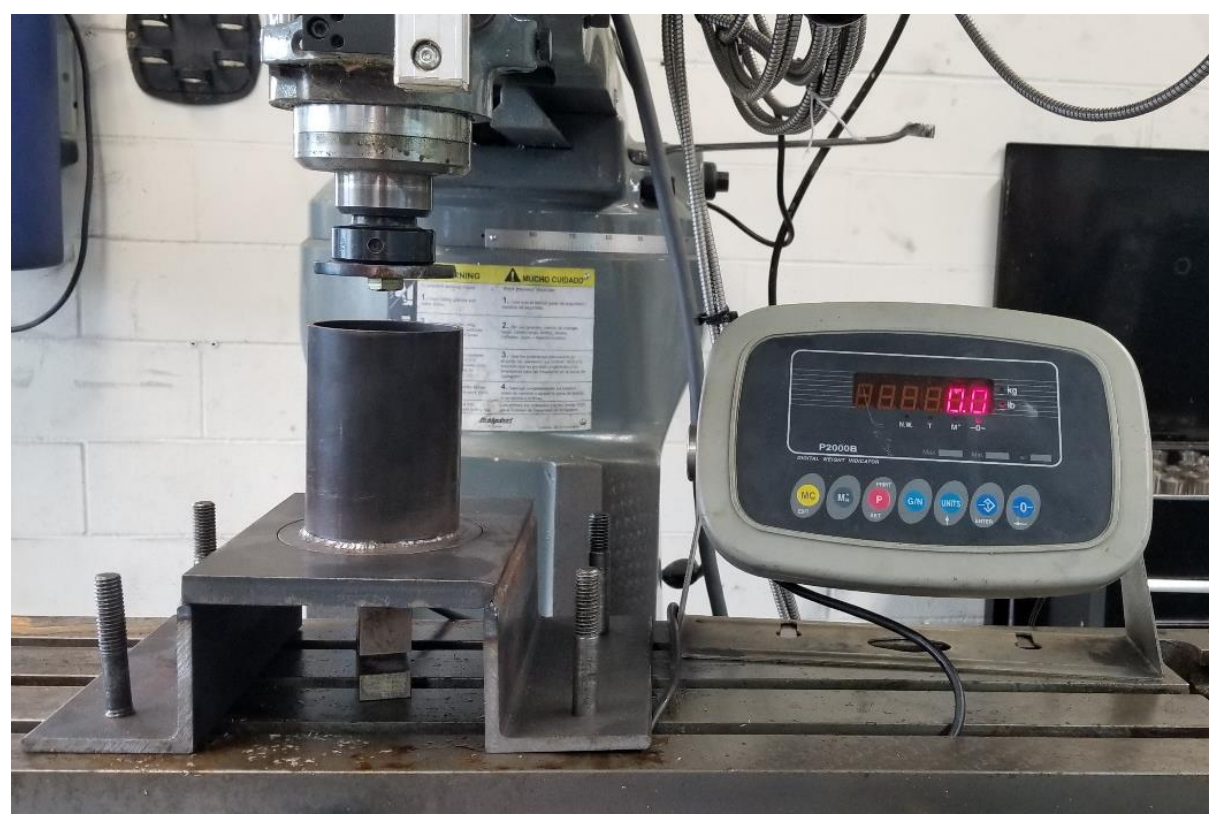

Figure 17: CVT Spring Linear Constant Test Setup.

Torsional testing required the top of the spring to be twisted without the bottom of the spring moving. To do this, the top and bottom spacers had a small hole cut in their outer edges for 
the spring flanges to work against. The bottom spacer was now threaded on a block of steel which was secured to the mill bed with a T-Nut. The top spacer had a short bolt threaded into its face opposite the stem. Finally, a torque wrench with a digital readout was used on this bolt to twist the torsional spring and observe the twist required to reach a specified torque reading.

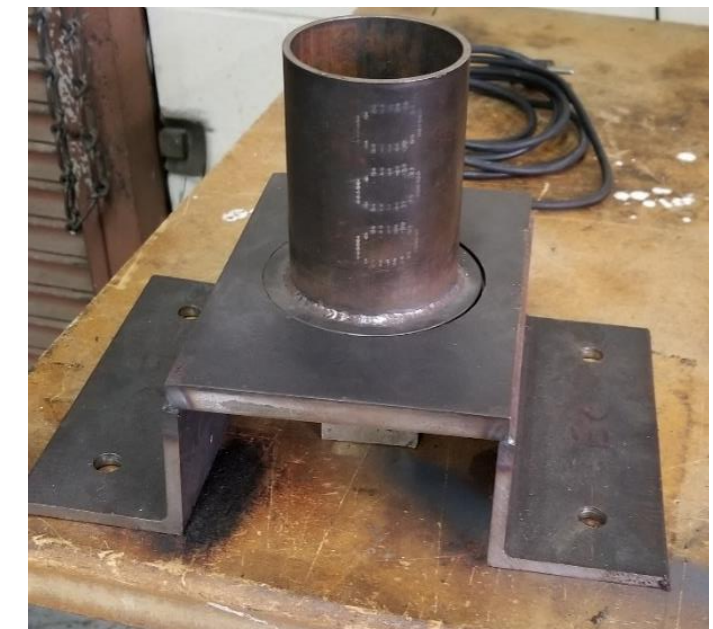

Figure 18: Torsional Spring Tester Picture 1.

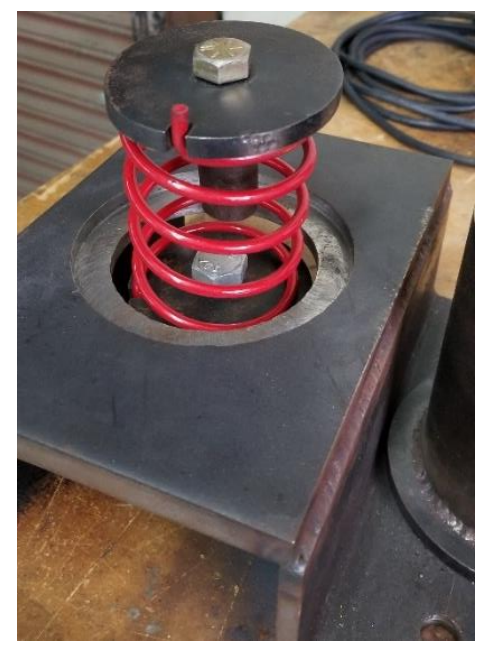

Figure 19: Torsional Spring Tester Picture 2.

\section{Linear Constants:}

To ensure accurate data, the spring was compressed in quarter inch increments, observing the force at each point. Data points were taken in this manner from zero to 1.5 inches displaced. The force at each point was divided by its associated displacement, revealing a spring constant to that point of compression. This process was repeated in ten trials and an overall average spring constant was calculated. The overall spring constant was found by fitting the data points for force at a given displacement to a line following the least squares method. This method finds the line that best fits the data by minimizing the sum of the error at any given point. The slope of this line is then the resultant spring constant. Figure 20, taken from the textbook Introduction to Linear Algebra (Strang), outlines the equation for following the method. $\mathrm{C}$ represents the $\mathrm{y}$ intercept of the line, which is zero for this case. D represents the slop of the line, which is the spring constant in this case. A is the vector of displacements. Lower case $\mathrm{b}$ is the vector or force resultants.

$$
\begin{aligned}
& \text { The line } C+D t \text { minimizes } e_{1}^{2}+\cdots+e_{m}^{2}=\|A \boldsymbol{x}-\boldsymbol{b}\|^{2} \text { when } A^{\mathrm{T}} A \widehat{\boldsymbol{x}}=A^{\mathrm{T}} \boldsymbol{b} \text { : } \\
& \qquad A^{\mathrm{T}} A \widehat{x}=A^{\mathrm{T}} \boldsymbol{b} \quad\left[\begin{array}{cc}
m & \sum t_{i} \\
\sum t_{i} & \sum t_{i}^{2}
\end{array}\right]\left[\begin{array}{c}
C \\
D
\end{array}\right]=\left[\begin{array}{c}
\sum b_{i} \\
\sum t_{i} b_{i}
\end{array}\right] .
\end{aligned}
$$

Figure 20: Least Squares Approximation Equation. 


\section{Torsional Constants:}

Torsional tests were performed in a similar manner to the linear tests. The spring was twisted in increments using a torque wrench. The angular displacement and torque required to move the spring were recorded, and the torsional constant was calculated. To confirm test data, constants were also calculated empirically using Equation 3. This equation was dependent on the wire thickness (d), Young's modulus (E), mean diameter (D), and number of active coils $\left(N_{a}\right)$.

$$
\kappa_{\text {Torsional }}=\frac{d^{4} E}{10.8 * D * N_{a}}
$$

Equation 3: Torsional Spring Constant. (Richard G. Budynas) 


\section{MATLAB Calculations:}

Calculations were performed in MATLAB to determine the optimal combination of CVT variables to achieve tuning objectives. This script uses several functions whose inputs change based on the operation conditions. The two conditions tested were shifting, and engagement conditions. The script loops through every possible setup combination and truncates any setup that doesn't stay within the design objectives. The code is setup to make calculations for any CVT assuming the relationship between sub systems doesn't change.

\section{Main Script:}

The main script is where constants and variables of the entire system are stored. Both are pulled from an excel document and stored as variables in MATLAB. This setup makes seamless transition between tuning one CVT to the next. This script also calls functions for different operating conditions. Calculations that are independent of operating conditions are calculated in this section and passed into the operating condition functions. These calculations are the shift ratio the effective coefficient of friction between the belt and sheaves, the belt wrap angle, and the torque load from the weight of the vehicle.

\section{Shift Ratio:}

The shift ratio is dependent on the pitch radii of the primary and secondary. This value does change while shifting but is not affected by any tunable components.

$$
S R=\frac{\text { Radius }_{\text {Secondary }}}{\text { Radius }_{\text {Primary }}}
$$

Equation 4: Shift Ratio Based on Sheave Radii.

\section{Effective Coefficient of Friction:}

The effective coefficient of friction is determined based on the nominal coefficient of friction and the sheave groove angle. For the GX9, the primary and secondary sheave angle is the same, so a single effective coefficient is determined. The nominal coefficient of friction was not provided by GE, so a common value for rubber V-belt CVTs was used. (Richard G. Budynas)

$$
\begin{gathered}
\mu_{\text {effective }}=\frac{\mu_{\text {Nominal }}}{\sin \left(\frac{\emptyset}{2}\right)} \\
\text { Equation 5: Effective Coefficient of Friction. }
\end{gathered}
$$

Wrap Angle:

The wrap angle is dependent on the pitch radii of the primary and secondary and the distance between their centers. $\alpha$ and $\beta$ refer to the belt wrap of the primary and secondary respectively. The calculation for each change based on which pulley has a larger working radius. The shift ratio was used to determine which equation should be used.

\section{If $\mathrm{SR} \geq 1$ :}


$\alpha($ Radians $)=\pi-2 * \sin ^{-1}\left(\frac{\text { Radius }_{\text {Secondary }}-\text { Radius }_{\text {Primary }}}{2 * \text { Center to Center }}\right)$

Equation 6: Wrap Angle at the Primary $S R \geq 1$. (Richard G. Budynas)

$\beta($ Radians $)=\pi+2 * \sin ^{-1}\left(\frac{\text { Radius }_{\text {Secondary }}-\text { Radius }_{\text {Primary }}}{2 * \text { Center to Center }}\right)$

Equation 7: Wrap Angle at the Secondary SR $\geq 1$. (Richard G. Budynas)

If $\mathrm{SR}<1$ :

$\alpha($ Radians $)=\pi+2 * \sin ^{-1}\left(\frac{\text { Radius }_{\text {Secondary }}-\text { Radius }_{\text {Primary }}}{2 * \text { Center to Center }}\right)$

Equation 8: Wrap Angle at the Primary SR<1. (Richard G. Budynas)

$\beta($ Radians $)=\pi-2 * \sin ^{-1}\left(\frac{\text { Radius }_{\text {Secondary }}-\text { Radius }_{\text {Primary }}}{2 * \text { Center to Center }}\right)$

Equation 9: Wrap Angle at the Secondary SR $<1$. (Richard G. Budynas)

Vehicle Torque Load on the CVT:

The torque load from the weight of the vehicle is dependent on the weight of the vehicle and the hill angle $(\gamma)$ the vehicle is on. The torque load is multiplied by the tire radius, and gear box ratio.

$$
\begin{gathered}
\tau_{\text {Vehicle }}(f t . * l b s)=W e i g h t_{\text {Vehicle }} * \sin (\gamma) * \text { Radius }_{\text {Tire }} * R_{\text {GearBox }} \\
\text { Equation 10: Vehicle Torque Loading on Secondary. }
\end{gathered}
$$




\section{Operating Conditions:}

These conditions define several aspects of how the CVT is currently running. Most of the calculations made are uniform throughout but key input variables change.

\section{Shift out Conditions:}

The script is designed to make calculations for shift out first, because a straight shift is the primary tuning objective. This means only a couple of variables need to be set. The desired angular velocity of the primary is set where the engine produces max power. Design horsepower is defined based on this angular velocity.

\section{Engagement Conditions:}

At the engagement point the sheaves are stationary so several variables need to be changed. The desired angular velocity of the engine is set where max torque is produced. Design horsepower is set based on this angular velocity. The pitch radii of the primary and secondary, flyweight working radius, helix angle, and shift ratio are all set to their initial conditions. Finally, the displacement of all springs is set to zero.

\section{Torque:}

The torque experienced at the primary and secondary is calculated in each operating condition before passing into the functions. Torque at the primary was calculated using the theoretical engine horsepower at the desired angular velocity (Briggs \& Stratton Racing). This theoretical horsepower was confirmed with dynamometer testing described in the section Testing:.

$$
\begin{gathered}
\tau_{\text {Primary }}(f t . * l b f)=\frac{H p_{\text {Primary }} * 5252}{\omega_{\text {Desired }}} \\
\text { Equation 11: Torque at the Primary. }
\end{gathered}
$$

Torque at the secondary was determined using the calculated torque at the primary and the shift ratio.

$$
\begin{gathered}
\tau_{\text {Secondary }}(f t . * l b f)=\tau_{\text {Primary }} * S R \\
\text { Equation 12: Torque at the Secondary. }
\end{gathered}
$$




\section{CVT Force Balance:}

There are several forces to consider when considering the CVT. To start, a free body diagram of a V-belt wrapping around a pulley was considered. This free body diagram is shown below in Figure 21. The only point loads are due the taut and slack side tensions in the belt, $T_{1}$ and $T_{0}$. All remaining forces on the belt are distributed uniformly across the angle of wrap, $\beta$. The distributed loading $F_{\text {Dist }}$ is due to the clamping force of the sheaves and the centrifugal force of the belt itself. The objective is to derive a relationship between the slack side tension and the clamping force. To do this a summation of forces was taken in the X-direction, Equation 13. $F_{\text {Total_X }}$ is the total distributed loading in the X-direction. $T_{1 \_X}$ and $T_{0 \_X}$ are the $\mathrm{X}$ components of the taut and slack side tension respectively.

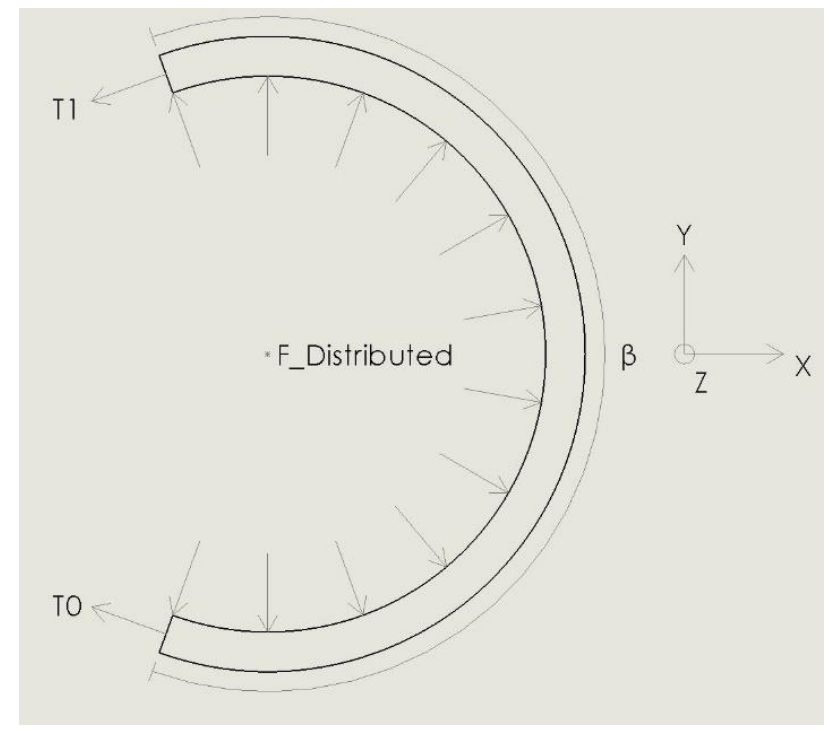

Figure 21: Belt Free Body Diagram.

$$
\begin{gathered}
\qquad F_{X}=0=F_{\text {Total_X }}-T_{1_{X}}-T_{0_{-} X} \\
\text { Equation 13: Belt Equation of Equilibrium X-Direction. }
\end{gathered}
$$

\section{Belt Tension:}

The tension on either side of the belt acts directly out from where the belt loses contact with the sheave. This point is determined by the wrap angle. Knowing this, a relationship can be determined between the $\mathrm{X}$ component of the tension and the current wrap angle. Consider another free body diagram of the belt, Figure 22, highlighting the angle of tension. In this figure $\theta$ represents the angle between the tension and the $\mathrm{X}$-axis. The relationship between this angle and the wrap angle changes once the wrap angle is greater than $\pi$. Equation 14 and Equation 15 detail the relationship for both cases. 


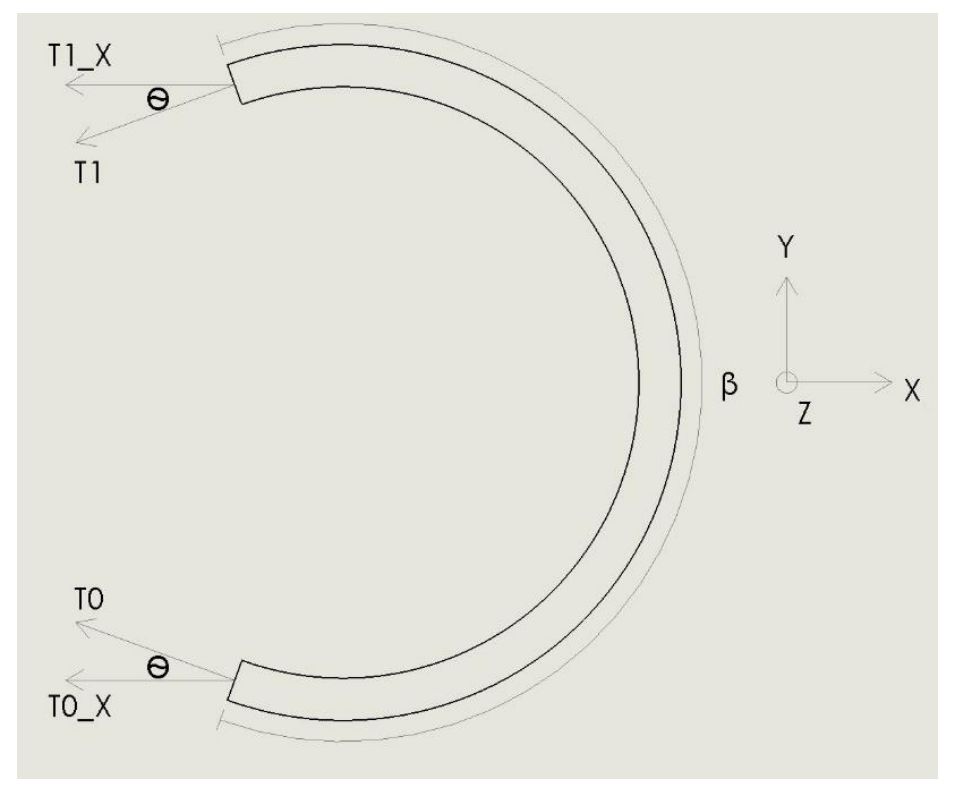

Figure 22: Belt Tension Free Body Diagram.

If $\beta \geq \pi$

$$
\theta=\frac{1}{2}(\beta-\pi)
$$

Equation 14: Tension Angle if $\beta \geq \pi$.

If $\beta \leq \pi$

$$
\theta=\frac{1}{2}(\pi-\beta)
$$

Equation 15: Tension Angle if $\beta \leq \pi$.

Now, the $\mathrm{X}$-component of these tensions can be written in terms of $\beta$.

$$
\begin{aligned}
& T_{0_{-} X}=T_{0} \cos (\theta) \\
& T_{1_{\_} X}=T_{1} \cos (\theta)
\end{aligned}
$$

The taut side tension can be written in terms of the slack side tension based on Euler's number and the effective coefficient of friction. This relation is detailed in Equation 16.

$$
T_{1}=T_{0} e^{\mu_{e} \beta}
$$

\section{Equation 16: Taut and Slack Side Tension Relationship.}

Using Equation 14, Equation 15, and Equation 16 the X-component of the taut and slack side tension is found in terms of the wrap angle and the overall slack side tension.

If $\beta \geq \pi$ 


$$
T_{0_{X}}(l b f)=T_{0} \cos \left(\frac{1}{2}(\beta-\pi)\right)
$$

Equation 17: Slack Side Tension X-Component if $\beta \geq \pi$.

$$
T_{1_{X}}(l b f)=T_{0} e^{\mu_{e} \beta} \cos \left(\frac{1}{2}(\beta-\pi)\right)
$$

Equation 18: Taut Side Tension X-Component if $\beta \geq \pi$.

If $\beta \leq \pi$

$$
T_{0_{X}}(l b f)=T_{0} \cos \left(\frac{1}{2}(\pi-\beta)\right)
$$

Equation 19: Slack Side Tension X-Component if $\beta \leq \pi$.

$$
T_{1_{X}}(l b f)=T_{0} e^{\mu_{e} \beta} \cos \left(\frac{1}{2}(\pi-\beta)\right)
$$

Equation 20: Taut Side Tension X-Component if $\beta \leq \pi$.

Total Force:

The distributed loading on the belt $F_{D i s t}$ is the summation of the centrifugal force, $C_{D i s t}$, and the radial component of the clamping force, $F_{\text {Radial }}$. This relationship is described in Equation 21.

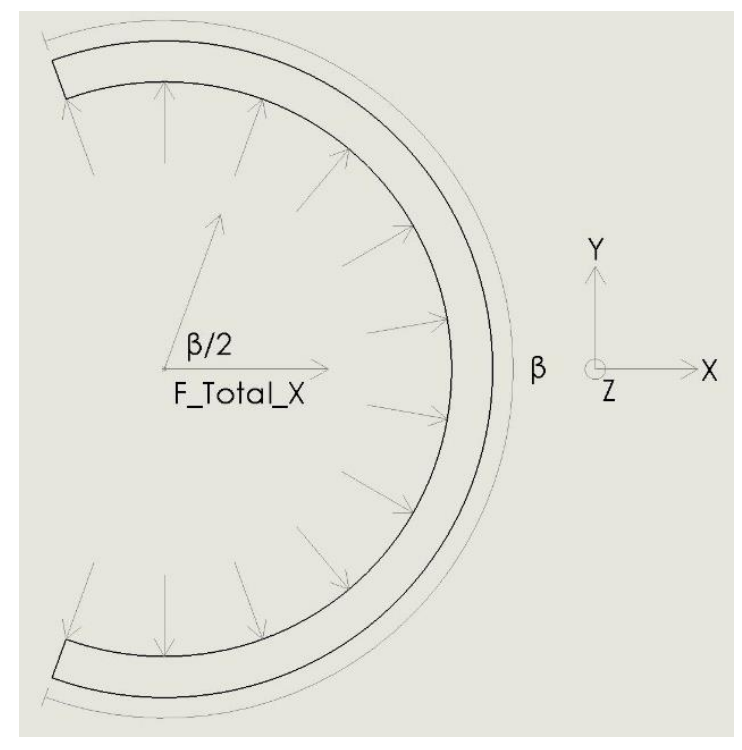

Figure 23: Belt Distributed Loading along wrap angle.

$$
F_{\text {Dist }}=F_{\text {Radial }}+C_{\text {Dist }}
$$

Equation 21: Total Distributed Loading.

From this distributed force, a total force $F_{\text {Total }}$, is found by integrating the distributed loading over the wrap angle. Additionally, to find the X-component of $F_{\text {Total }}$ the cosine of the 
distributed force must be taken at each point along the wrap angle. At any wrap angle $\beta$ the angle between the distributed force and the $\mathrm{X}$-axis is $\frac{\beta}{2}$.

$$
\begin{gathered}
F_{\text {Total_X }}=2 \int_{0}^{\frac{\beta}{2}} F_{\text {Dist }} * \cos \left(\frac{\beta}{2}\right) d \frac{\beta}{2} \\
F_{\text {Total_X }}=2 F_{\text {Dist }} \sin \left(\frac{\beta}{2}\right)
\end{gathered}
$$

Substituting Equation 21 in for $F_{\text {Dist }}$ yields Equation 22 for the total force in the Xdirection.

$$
\begin{gathered}
F_{\text {Total }_{X}}(l b f)=2 \sin \left(\frac{\beta}{2}\right) *\left(F_{\text {Radial }}+C_{\text {Dist }}\right) \\
\text { Equation 22: Total Force in the X-Direction. }
\end{gathered}
$$

\section{Centrifugal Force:}

The centrifugal force works to pull the belt out of the sheaves because it is rotating mass. This force is dependent on the mass per unit length $\left(M_{B e l t}\right)$, the pitch radius $(\mathrm{R})$, and the angular velocity $(\omega)$. The general formula for centrifugal force $(C)$ is written in Equation 23 where $M_{\text {Total }}$ is the total mass rotating.

$$
\begin{gathered}
\qquad C=M_{\text {Total }} * \omega^{2} * R \\
\text { Equation 23: Centrifugal Belt Force. }
\end{gathered}
$$

The total mass changes as the CVT shifts because the length of belt in contact with the sheaves changes. It is more useful to write the centrifugal force in terms of the angle of wrap. This is done by relating the total mass to the mass per unit length and the arc length in

$$
\begin{aligned}
& M_{\text {Total }}=M_{\text {Belt }} * R * \beta \\
& \text { Equation 24: Total Belt Mass. }
\end{aligned}
$$

Substituting Equation 24 into Equation 23 yields the total centrifugal force in terms of $\beta$.

$$
\begin{aligned}
& C=\frac{1}{12} M_{\text {Belt }} * R^{2} * \omega^{2} * \beta \\
& \text { Equation 25: Total Centrifugal Force. }
\end{aligned}
$$

Dividing Equation 25 by the total wrap angle yields the distributed centrifugal force.

$$
C_{\text {Dist }}\left(\frac{l b f}{r a d}\right)=\frac{1}{12} M_{\text {Belt }} * R^{2} * \omega^{2}
$$




\section{Radial Force:}

To find the radial force due to clamping, consider a free body diagram of the belt cross section (Figure 24). In this FBD the fixed sheave is on the right, while the moveable sheave is on the left. The fixed sheave acts as a fixed wall in this force analysis. The objective is to derive the relationship between the clamping force, $F_{\text {Clamp }}$ and the radial force it causes $F_{\text {Radial }}$. R represents the reaction from the fixed sheave due to the clamping force. $\phi$ is the sheave groove angle. For ease of writing equations, let $F_{\text {Clamp }}=F_{z}$ and $F_{\text {Radial }}=A_{y^{\prime}}$. To find $A_{y^{\prime}}$ in terms of $F_{z}$ a summation of forces in the $\mathrm{y}^{\prime}$ direction was taken. This equation of equilibrium is written in

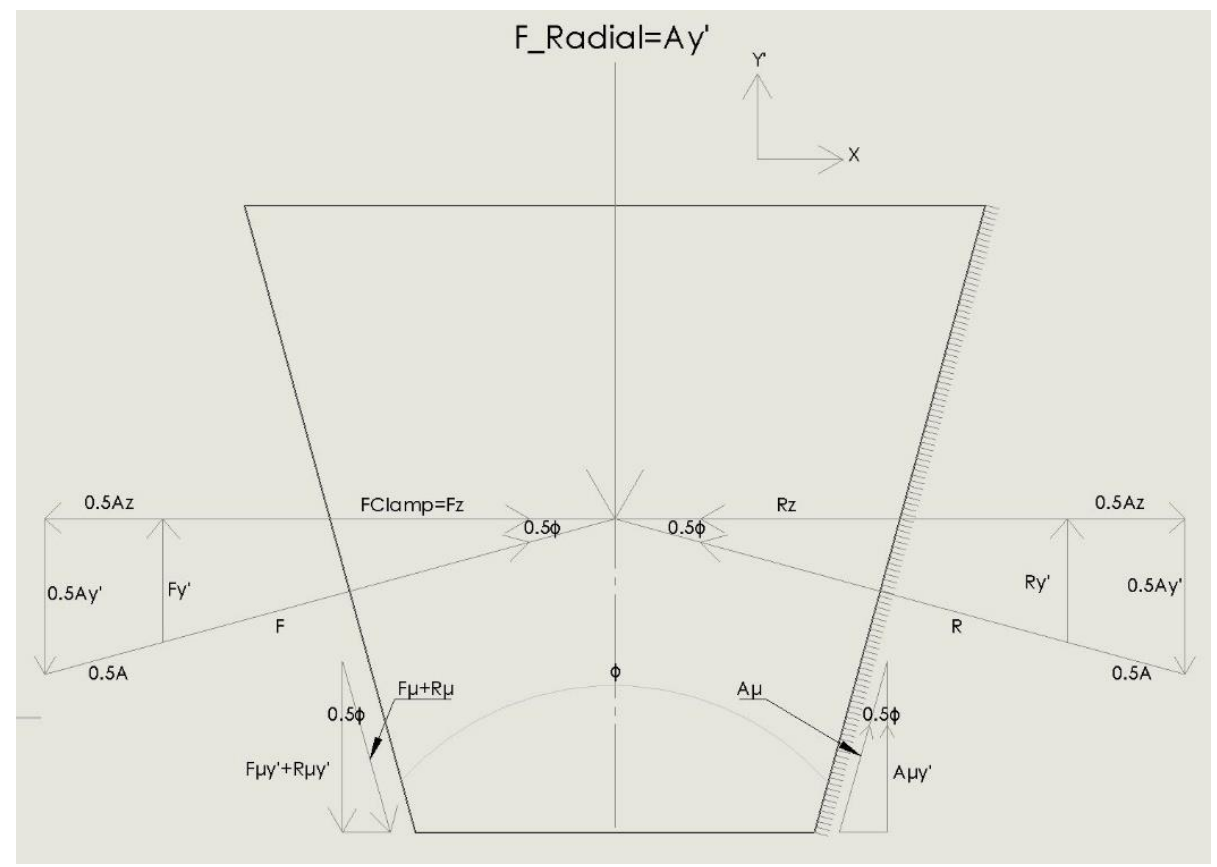

Figure 24: Belt Cross Section Free Body Diagram.

$$
\Sigma F_{y^{\prime}}=0=F_{y^{\prime}}+R_{y^{\prime}}-A_{y^{\prime}}-F_{\mu y^{\prime}}-R_{\mu y^{\prime}}+A_{\mu y \prime}
$$

Equation 27: Belt Cross Section Sum of Forces in Y'.

$\mathrm{R}$ is the reaction on the belt from the fixed sheave. $A_{y^{\prime}}$ acts in the $\mathrm{y}^{\prime}$ direction, therefore:

$$
F_{z}=R_{z}
$$

From this relationship Equation 27 can be rewritten:

$$
\begin{aligned}
& \Sigma F_{y^{\prime}}=0=2 F_{y^{\prime}}-A_{y^{\prime}}-2 F_{\mu y^{\prime}}+A_{\mu y \prime} \\
& \text { Equation 28: Belt Cross Section Sum of Forces in } Y^{\prime} .
\end{aligned}
$$

$F_{y}$ is related to $F_{z}$ based on the belt groove geometry:

$$
F_{y^{\prime}}=F_{z} \tan \left(\frac{\phi}{2}\right)
$$


$F_{\mu y^{\prime}}$ is the y' component of the friction force due to $F_{z}$ :

$$
F_{\mu y^{\prime}}=F_{\mu} \cos \left(\frac{\phi}{2}\right)=F \mu_{s} \cos \left(\frac{\phi}{2}\right)=\frac{F_{z}}{\cos \left(\frac{\phi}{2}\right)} \mu_{s} \cos \left(\frac{\phi}{2}\right)
$$

This can be simplified to Equation 30:

$$
F_{\mu y^{\prime}}=F_{z} \mu_{s}
$$

Equation 30: Friction Due to Clamping Force in $Y^{\prime}$.

The friction due to the radial force is found using a similar approach:

$$
\mu_{A y^{\prime}}=\mu_{A} \cos \left(\frac{\phi}{2}\right)=A \mu_{s} \cos \left(\frac{\phi}{2}\right)=\frac{A_{y^{\prime}} \mu_{s} \cos \left(\frac{\phi}{2}\right)}{\sin \left(\frac{\phi}{2}\right)}
$$

Simplifying yields Equation 31:

$$
\begin{gathered}
A_{\mu y \prime}=A_{y^{\prime}} \mu_{s} \cot \left(\frac{\phi}{2}\right) \\
\text { Equation 31: Friction Due to Radial Force in } Y^{\prime} \text {. }
\end{gathered}
$$

Substituting Equation 29, Equation 30, and Equation 31 into Equation 28:

$$
2 F_{z} \tan \left(\frac{\phi}{2}\right)-A_{y^{\prime}}-2 F_{z} \mu_{s}+A_{y^{\prime}} \mu_{s} \cot \left(\frac{\phi}{2}\right)=0
$$

Rearranging for $A_{y^{\prime}}$ and simplifying yields:

$$
\begin{gathered}
A_{y^{\prime}}=2 F_{z} \tan \left(\frac{\phi}{2}\right) \\
\text { Or } \\
F_{\text {Radial }}\left(\frac{l b f}{r a d}\right)=2 F_{\text {Clamp }} \tan \left(\frac{\phi}{2}\right)
\end{gathered}
$$

Equation 32: Radial Component of the Clamping Force.

Force Balance:

All forces have now been written in terms of the clamping force, wrap angle, and constants of the system. Substituting Equation 32 and Equation 26 into Equation 22 yields an expression for the total force in the X-direction: 


$$
F_{\text {Total }_{X}}(l b f)=2 \sin \left(\frac{\beta}{2}\right) *\left[2 F_{\text {Clamp }} \tan \left(\frac{\phi}{2}\right)+\frac{1}{12} M_{\text {Belt }} * R^{2} * \omega^{2}\right]
$$

This expression, along with Equation 17, Equation 18, Equation 19, and Equation 20 can be substituted into Equation 13 to yield two cases.

$$
\begin{aligned}
& \text { If } \beta \geq \pi \\
& 2 \sin \left(\frac{\beta}{2}\right) *\left[2 F_{\text {Clamp }} \tan \left(\frac{\phi}{2}\right)+\frac{1}{12} M_{\text {Belt }} * R^{2} * \omega^{2}\right]=T_{0} e^{\mu_{e} \beta} \cos \left(\frac{1}{2}(\beta-\pi)\right)+T_{0} \cos \left(\frac{1}{2}(\beta-\pi)\right) \\
& \text { If } \beta \leq \pi \\
& 2 \sin \left(\frac{\beta}{2}\right) *\left[2 F_{\text {Clamp }} \tan \left(\frac{\phi}{2}\right)+\frac{1}{12} M_{\text {Belt }} * R^{2} * \omega^{2}\right]=T_{0} e^{\mu_{e} \beta} \cos \left(\frac{1}{2}(\pi-\beta)\right)+T_{0} \cos \left(\frac{1}{2}(\pi-\beta)\right)
\end{aligned}
$$

Rearranging these equations and solving for $T_{0}$ yields Equation 33 and Equation 34. These expressions relate the slack side tension to the clamping force at either pulley. They are the governing equations used for all calculations at the primary and secondary.

If $\beta \geq \pi$

$$
T_{0}(l b f)=\frac{2 \sin \left(\frac{\beta}{2}\right) *\left[2 F_{\text {Clamp }} \tan \left(\frac{\phi}{2}\right)+\frac{1}{12} M_{\text {Belt }} * R^{2} * \omega^{2}\right]}{\cos \left(\frac{1}{2}(\beta-\pi)\right) *\left(e^{\mu_{e} \beta}+1\right)}
$$

Equation 33: Slack Side Tension if $\beta \geq \pi$.

If $\beta \leq \pi$

$$
T_{0}(\text { lbf })=\frac{2 \sin \left(\frac{\beta}{2}\right) *\left[2 F_{\text {Clamp }} \tan \left(\frac{\phi}{2}\right)+\frac{1}{12} M_{\text {Belt }} * R^{2} * \omega^{2}\right]}{\cos \left(\frac{1}{2}(\pi-\beta)\right) *\left(e^{\mu_{e} \beta}+1\right)}
$$




\section{Secondary Function:}

This function performs all calculations for the secondary clutch. The objective is to calculate the tension in the belt caused by the clamping force. Clamping force is due to the torsional spring forces, and torque feedback from the helix.

\section{Torsional Spring:}

The compression force of this spring is dependent on the installed length, linear spring constant, and how far the sheave has shifted.

$$
\begin{gathered}
F_{\text {Spring }_{\text {Linear }}}(l b f)=\kappa_{\text {Linear }} *\left(X_{\text {Installed }}+X_{\text {Shift }}\right) \\
\text { Equation 35: Linear Component of Spring Force. }
\end{gathered}
$$

The torsional force of this spring is dependent on the installed twist, torsional spring constant, and how far the sheave has twisted.

$$
\begin{gathered}
F_{\text {Spring_Torsional }}(l b f)=\kappa_{\text {Torsional }} *\left(\lambda_{\text {Installed }}+\lambda_{\text {Shift }}\right) \\
\text { Equation 36: Torsional Component of Spring Force. }
\end{gathered}
$$

Helix:

The force on the helix is determined from the torsional spring force and torque feedback. The torque feedback is dependent on the torque at the secondary and the helix radius from the center line. Summing these two forces gives the total force working on the helix. A percentage of this force becomes clamping force based on the angle of the helix. This relationship is detailed below in Figure 25.

$$
\begin{gathered}
F_{\text {Torque }}(\text { lbf })=\frac{\tau_{\text {Secondary }} * 12}{\text { Radius }_{\text {Helix }}} \\
\text { Equation 37: Helix Force Due to Torque Loading. } \\
F_{\text {Helix }}(\text { lbf })=\frac{\left(F_{\text {Spring_Torsional }}+F_{\text {Torque }}\right)}{2 * \tan (\eta)} \\
\text { Equation 38: Clamping Force From the Helix. (Aaen) }
\end{gathered}
$$

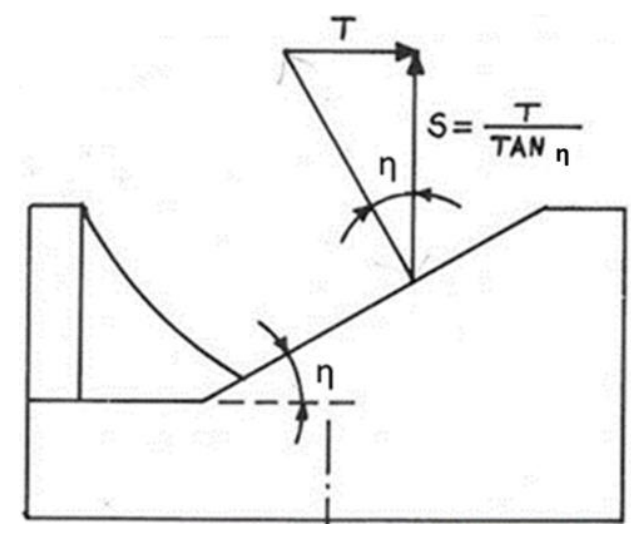

Figure 25: Secondary Helix FBD. (Aaen) 


\section{Clamping Force:}

Summing the linear spring force and helix force gives the overall clamping force of the secondary. This force is uniform over the belt wrap angle $(\beta)$ yielding Equation 39, the distributed clamping force. This value is then substituted into either Equation 33 or Equation 34 to solve for the slack side tension. Once the slack side tension is found, Equation 16 is used to find the taut side tension.

$$
\begin{gathered}
F_{\text {Clamp }}\left(\frac{l b f}{\text { rad }}\right)=\frac{F_{\text {Spring_Linear }}+F_{\text {Helix }}}{\beta} \\
F_{\text {Clamp }}\left(\frac{l b f}{\text { rad }}\right)=\frac{\kappa_{\text {Linear }} *\left(X_{\text {Installed }}+X_{\text {Shift }}\right)+\frac{\left(\kappa_{\text {Torsional }} *\left(\lambda_{\text {Installed }}+\lambda_{\text {Shift }}\right)+\frac{\tau_{\text {Secondary }} * 12}{\text { Radius }_{\text {Helix }}}\right)}{2 * \tan (\eta)}}{\beta}
\end{gathered}
$$

Equation 39: Distributed Clamping Force at the Secondary.

\section{Maximum Transferrable Torque:}

The maximum torque transferrable without slipping is determined based on the pitch radius and the difference in taut and slack tension (PSU). In a perfectly efficient system, the maximum torque transferrable would be just greater than the torque received. The efficiency of the secondary is determined by comparing these two values.

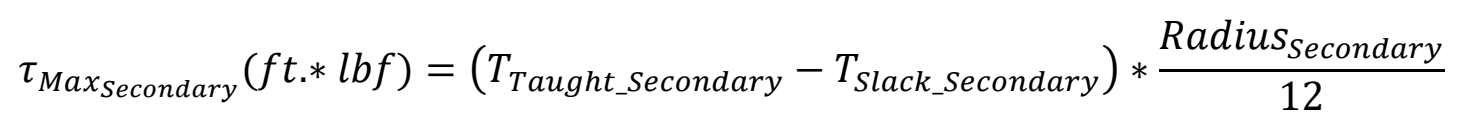




\section{Primary Function:}

This function performs all calculations for the primary clutch. The objective is to take the known tension in the belt and determine the clamping force required to balance it. From this required clamping force, the required angular velocity of the motor is determined. The clamping force is dependent on the spring force and flyweight force.

\section{Belt Tension:}

For shifting to occur the primary must be creating enough tension to pull the belt into the secondary. This means that the desired taut side tension at the primary must be just equal to the taut side tension at the secondary. The slack side tension is found by rearranging Equation 16 and using the belt wrap at the primary.

$$
\begin{gathered}
T_{1 \_ \text {Primary }}(l b f)=T_{1 \_ \text {Secondary }} \\
\text { Equation 41: Taut Side Tension at the Primary. } \\
\qquad T_{0_{\text {Primary }}}(l b f)=\frac{T_{1 \_ \text {Primary }}}{e^{\mu_{e} \alpha}} \\
\text { Equation 42: Slack Side Tension at the Primary. }
\end{gathered}
$$

Now Equation 33 or Equation 34 is rearranged to solve for $F_{\text {Clamp }}$ and Equation 42 is substituted in for $T_{0}$. This yields the required clamping force for shifting to occur, $F_{\text {Clamp_Required }}$. Pressure Spring:

The pressure spring force is determined based on the spring constant, installed compression, and distance shifted.

$$
\begin{gathered}
F_{\text {Spring }}(l b f)=\kappa_{\text {Linear }} *\left(X_{\text {Installed }}+X_{\text {Shift }}\right) \\
\text { Equation 43: Force From the Pressure Spring. }
\end{gathered}
$$

Flyweight Centrifugal Force:

The clamping force required by the flyweight mechanism is the summation of the required clamping force on the belt and the force from the pressure spring (Equation 44). The total centrifugal force of the flyweight system is based on the force of the flyweight, $F_{C}$, and the link it is mounted on, $L_{C}$. The relationship between centrifugal force and clamping force produced is based on the ramp geometry. A free body diagram of this system is detailed below in Figure 26.

$$
\begin{gathered}
F_{\text {Fly }} \text { Clamp } \\
\text { Equation 44: Clamping Force Due to the Flyweights. }
\end{gathered}
$$




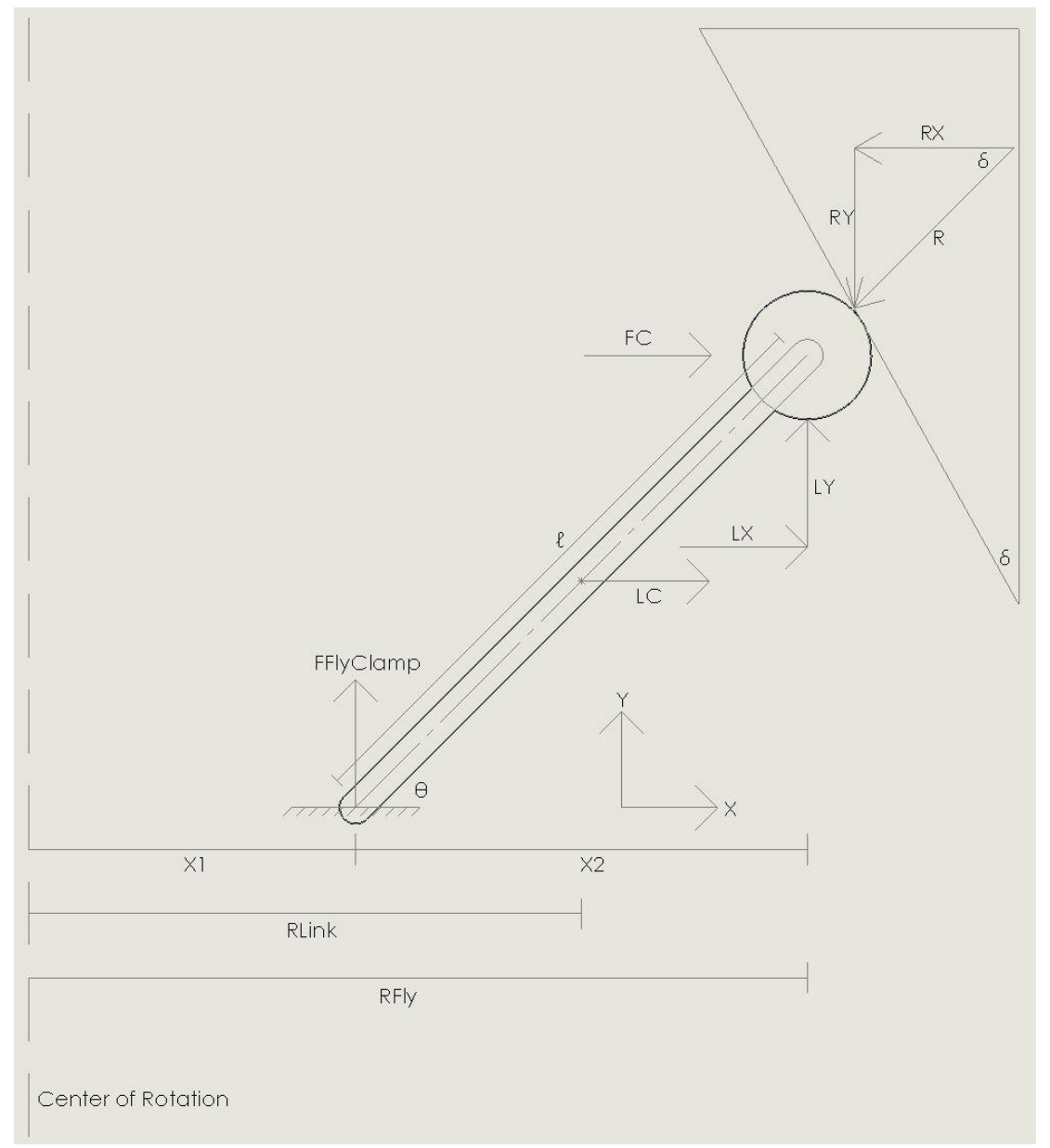

Figure 26: Flyweight System Free Body Diagram.

$R_{F l y}, X_{1}, 1$, and $\delta$ are known constants. They can be used to solve for other variables in the system. The angle between the link and the ground is found using Equation 45.

$$
\theta=\cos ^{-1}\left(\frac{R_{F l y}-X_{1}}{l}\right)
$$

Equation 45: Link Angle Geometry.

$R_{\text {Link }}$ is the distance from the center of rotation to the center of mass of the link. It is found using Equation 46.

$$
R_{\text {Link }}(\text { in })=X_{1}+\frac{l}{2} \cos (\theta)
$$

Equation 46: Link Radius Geometry.

Now, a summation of forces in $\mathrm{X}$ and $\mathrm{Y}$ is taken to relate the required forces to the centrifugal forces of the flyweight and the link.

$$
\Sigma F_{Y}=0=L_{Y}-R_{Y}
$$




$$
\begin{gathered}
\Sigma F_{X}=0=F_{C}+L_{C}+L_{X}-R_{X} \\
\text { Equation 48: Flyweight System Sum of Forces in } Y .
\end{gathered}
$$

The force in the y direction of the link pushing on the ramp is equal to the required flyweight system clamping force. Based on Equation 47 this is then equal to the reaction in the $y$ direction as well.

$$
R_{Y}=L_{Y}=F_{\text {Fly }} \text { Clamp }
$$

Now, $R_{X}$ and $L_{X}$ can be written in terms of $F_{\text {Fly }}$ Clamp

$$
\begin{gathered}
R_{X}(l b f)=\frac{R_{Y}}{\tan (\delta)}=\frac{F_{F l y_{\text {Clamp }}}}{\tan (\delta)} \\
\text { Equation 49: Flyweight System Reaction in the X-Direction. } \\
L_{X}(l b f)=\frac{L_{Y}}{\tan (\theta)}=\frac{F_{F l y_{\text {Clamp }}}}{\tan (\theta)}=\frac{F_{\text {Fly }} \text { Clamp }}{\tan \left(\cos ^{-1}\left(\frac{R_{F l y}-X_{1}}{l}\right)\right)}
\end{gathered}
$$

Equation 50: Flyweight System Link Force in the X-Direction.

Substituting Equation 49 and Equation 50 into Equation 48 yields the centrifugal force in terms of the required flyweight system clamping force.

$$
F_{C}+L_{C}=\frac{F_{F l y_{C l a m p}}}{\tan (\delta)}+\frac{F_{F l y_{C l a m p}}}{\tan \left(\cos ^{-1}\left(\frac{R_{F l y}-X_{1}}{l}\right)\right)}
$$

Equation 51: Flyweight System Required Centrifugal Force.

\section{Engine Speed:}

The engine speed required to create this centrifugal force is dependent on the working radius and mass of the flyweights and link. The centrifugal forces can be rewritten in terms of their mass and working radii:

$$
\begin{gathered}
F_{C}(l b f)=M_{F l y} * R_{F l y} * \omega^{2} \\
\text { Equation 52: Flyweight Centrifugal Force. } \\
L_{C}(l b f)=M_{\text {Link }} * R_{\text {Link }} * \omega^{2}=M_{\text {Link }} *\left(X_{1}+\frac{l}{2} \cos (\theta)\right) * \omega^{2} \\
\text { Equation 53: Link Centrifugal Force. }
\end{gathered}
$$

Substituting Equation 52 and Equation 53 into Equation 51 and rearranging to solve for $\omega$ yields Equation 54, the required engine speed. 


$$
\begin{gathered}
\omega_{\text {Primary }}(R P M)=\sqrt{\frac{F_{F l y_{\text {Clamp }}}}{M_{F l y} * R_{F l y}+M_{\text {Link }} * R_{\text {Link }}} *\left(\frac{1}{\tan (\delta)}-\frac{1}{\tan \left(X_{1}+\frac{l}{2} \cos (\theta)\right)}\right)} \\
\text { Equation 54: Engine Speed Based on Required Clamping Force. }
\end{gathered}
$$

Maximum Torque Transferrable:

The maximum torque transferrable without slipping is calculated to ensure power transfer with minimal slipping.

$$
\begin{gathered}
\tau_{\text {Max } \text { Primary }}(f t . * l b f)=\left(T_{\text {Taught_Primary }}-T_{\text {Slack_Primary }}\right) * \frac{\text { Radius }_{\text {Primary }}}{12} \\
\text { Equation 55: Maximum Torque Transferrable Primary. (PSU) }
\end{gathered}
$$




\section{Finite Element Analysis:}

Tension on the belt is the single variable that connects the primary to the secondary. Balancing this force properly is the root of controlling CVT performance. As a method of confirming calculations performed in MATLAB, an FEA model was developed to predict stress in the belt. From this value, tension in the belt was calculated and compared to the value calculated in MATLAB.

\section{SolidWorks Model:}

First, a CAD model of the CVT was created using SolidWorks. This was done by taking measurements off the GX9 and reflecting them on the model. This model was then imported into ANSYS Workbench for analysis. The full system is shown in

Figure 27. The CAD model consists of 3 sub-assemblies: the primary, secondary and the belt. Specific constraints were used to match motion of the model to the GX9. This includes sliding of the sheaves, clutch rotation, twisting at the secondary, and belt movement.

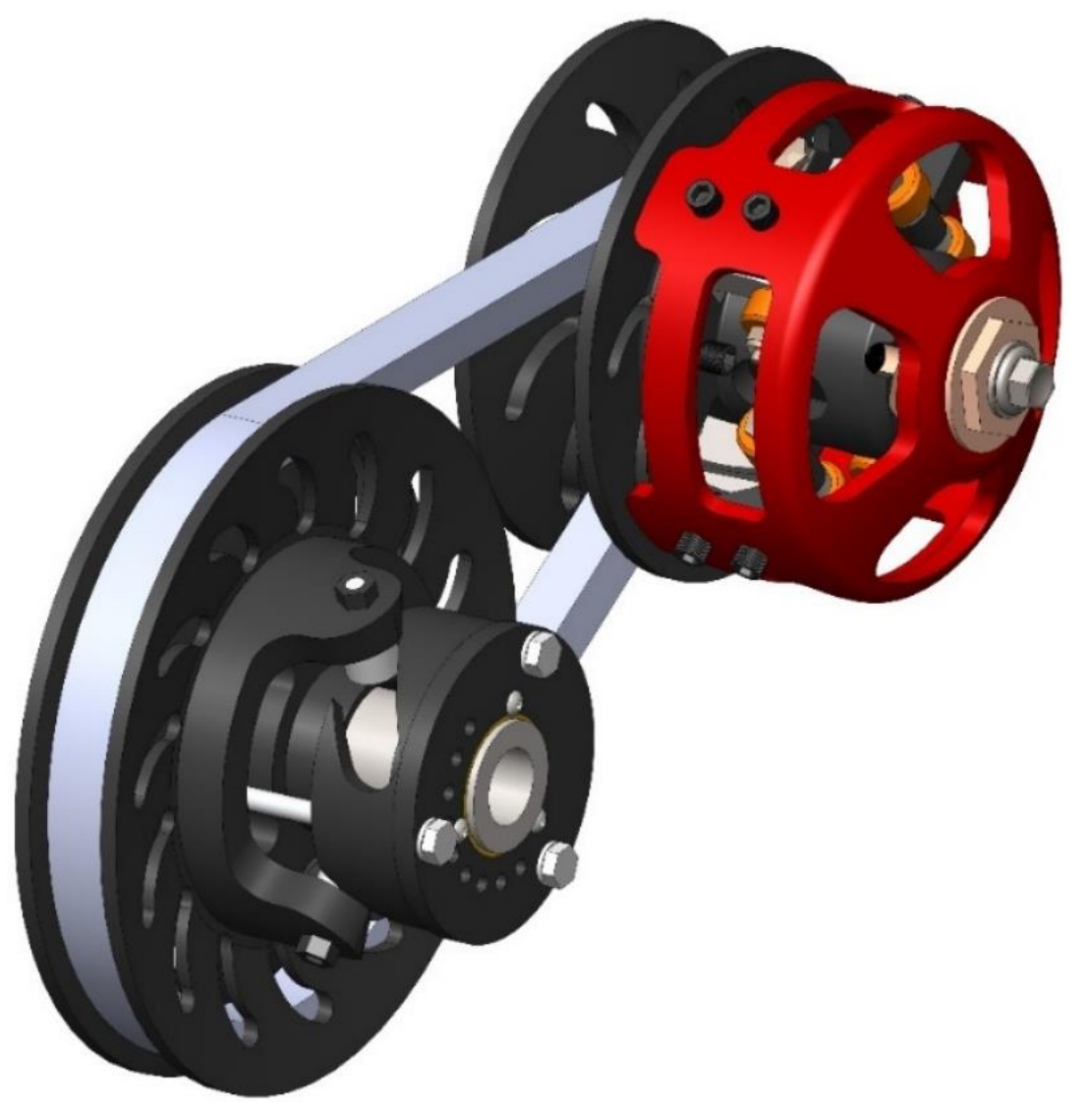

Figure 27: CVT System Assembly Made in SolidWorks. 
Figure 28 and Figure 29 show the primary and secondary respectively. The outer housing of the primary is transparent to highlight the flyweight mechanism. Constraints were placed on it such that as the moveable sheave shifts out the flyweights slide down the ramps. The secondary is angled to highlight the helix ramps. Constraints were placed on the roller bearings such that as the moveable sheave shifts it also twists, following the helix angle.

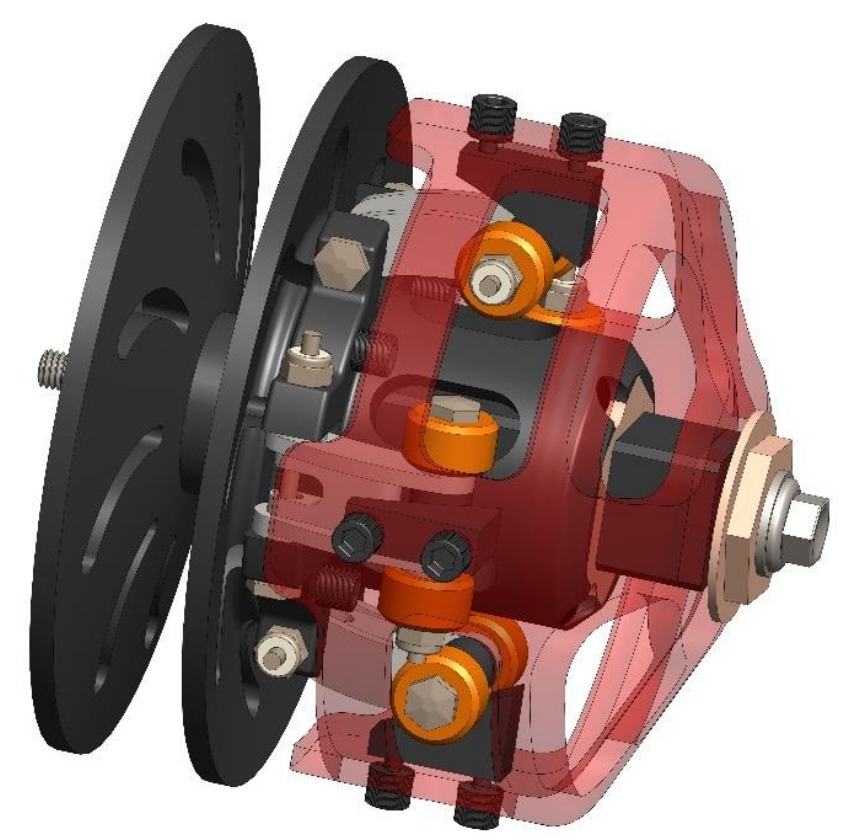

Figure 28: Primary Sub-Assembly Made in SolidWorks.

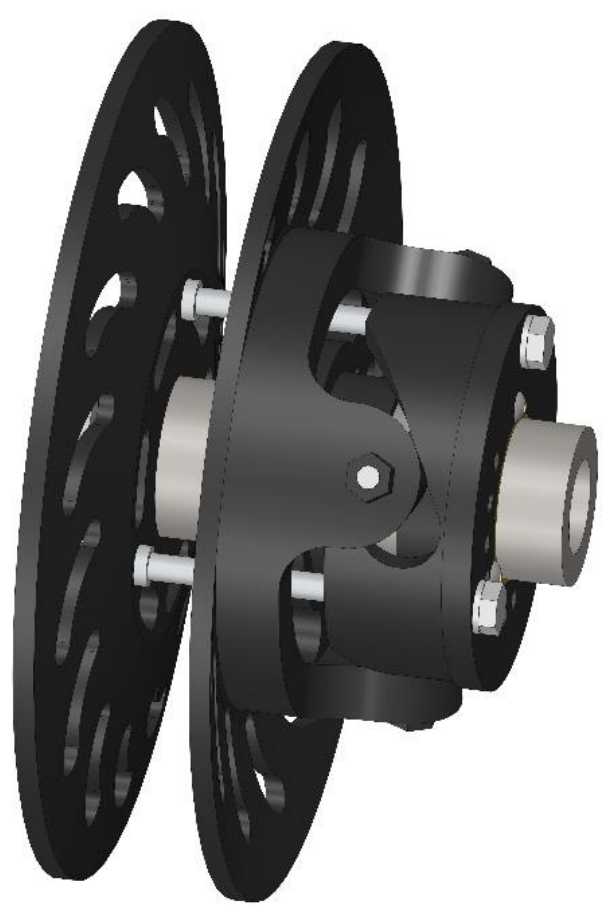

Figure 29: Secondary Sub-Assembly Made in SolidWorks. 
Figure 30 shows the belt model around the secondary. The belt itself was created by lofting a sketch of its cross section. The path for this loft is defined by a 3-D sketch of two circles. These circles represent the working radius of each clutch. Constraints were put in place to alter the diameter of these circles, so the belt moves in and out of the sheaves while shifting.

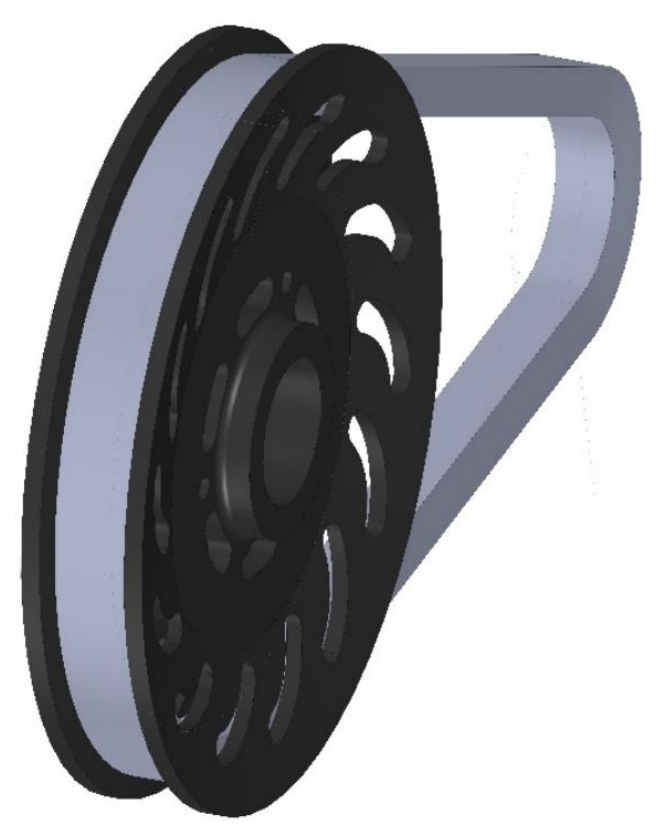

Figure 30: Belt Model Made in SolidWorks.

\section{ANSYS Workbench Model:}

Finite element analysis testing was conducted using ANSYS Workbench 18. The assembly created in SolidWorks was manipulated to represent the CVT in both low and high ratio. After the geometry was properly oriented, the belt was converted into a STEP file and imported into ANSYS. This ensured that details such as the wrap angle and contact area were correctly represented. Due to the loft tool used in SolidWorks, the belt breaks up into three distinct sections. These sections separate at where the belt is no longer in contact with the sheave surface. This is important for determining the contact area between the belt and sheave surface at different shift points. These sections are highlighted below in Figure 31. 


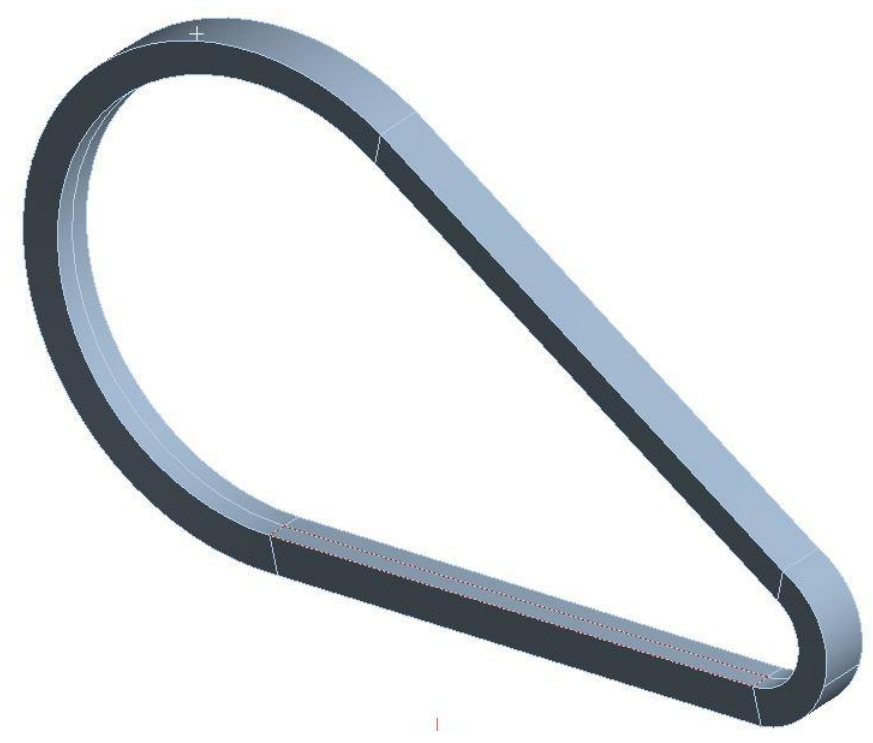

Figure 31: Belt Model Highlighting Section Division.

The next step was to define a few material properties of the belt namely the Young's Modulus of elasticity and the poisons ratio. The belt being represented is the Enduro 100 cogged V-Belt, the standard belt used for the GX9. There is no information for the belt's material properties listed on GE's website, so some measurements and estimations had to be made. The density of the belt was determined by measuring the belts cross sectional area, length, and weight. The Young's modulus and poisons ratio were estimated based on properties of a CVTech belt. This is another common. Figure 32 and Figure 33 show a CVTech and Enduro belt respectively. Material properties for the CVTech belt were found in a master's thesis from Virginia Polytech University. Dynamic mechanical analysis (DMA) was performed on several CVTech belts to determine material properties. This type of analysis allows loads to be applied at high frequency and high strain rates, representing the loading seen when in assembly (Messick). This study found a Young's Modulus of $13.8 \mathrm{ksi}$ and a Shear Modulus of $5.08 \mathrm{ksi}$. From these values ANSYS was able to calculate all other material properties. 


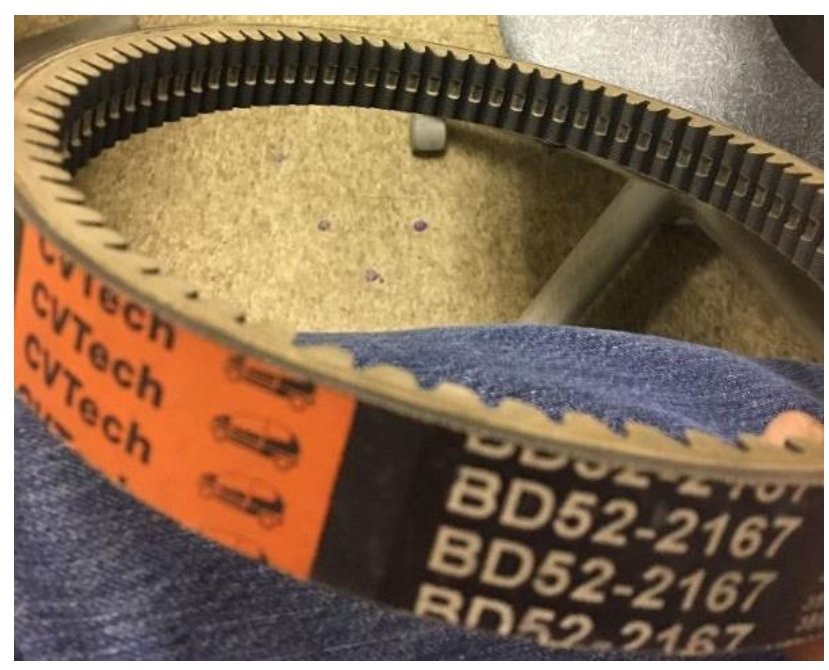

Figure 32: CVTech Belt. (Pennslyvania Tech)

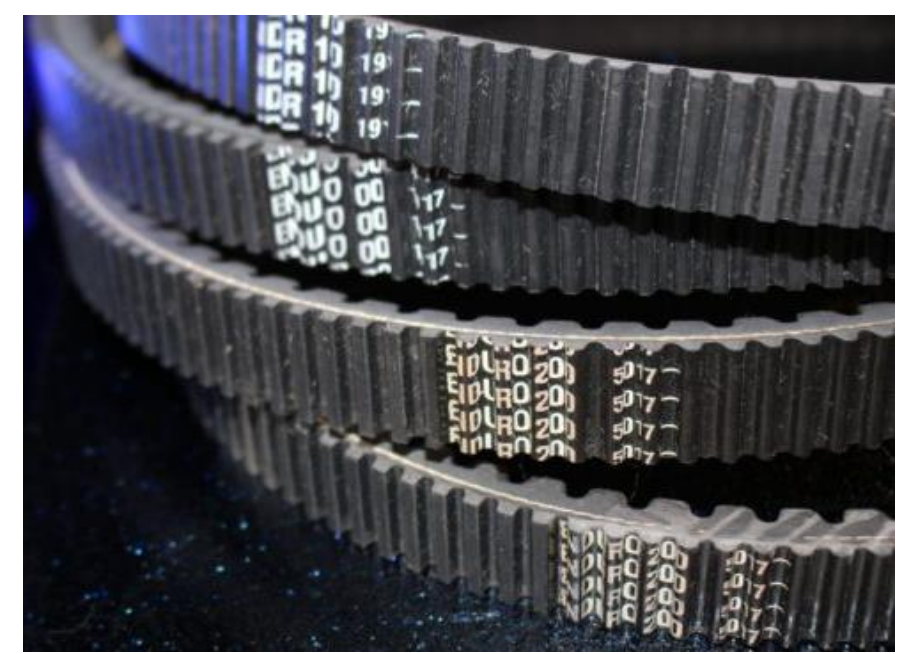

Figure 33: Enduro Belt. (Gaged Engineering)

After the geometry was imported, and material properties were defined, the force simulation was setup. Clamping force at the primary and secondary was determined at two shift points, low ratio and high ratio. These forces were applied onto their respective belt sections in the direction of that clutches moveable sheave (Figure 34). This implies that the forces operate on faces opposite each other. The face across from the applied force represents the fixed sheave. To represent this, that face was fixed in space in the simulation. Finally, the equivalent Von Mises Stress was determined across the entire belt in psi. The product of this stress and the area of the outer face yields the average tension around that sheave. This value was then compared to the average tension around the sheave calculated in MATLAB.

$$
T_{\text {Average }}=\sigma_{\text {Equivalent }} * \text { Area }_{\text {Face }}
$$

Equation 56: Average Tension Along the Wrap Angle.

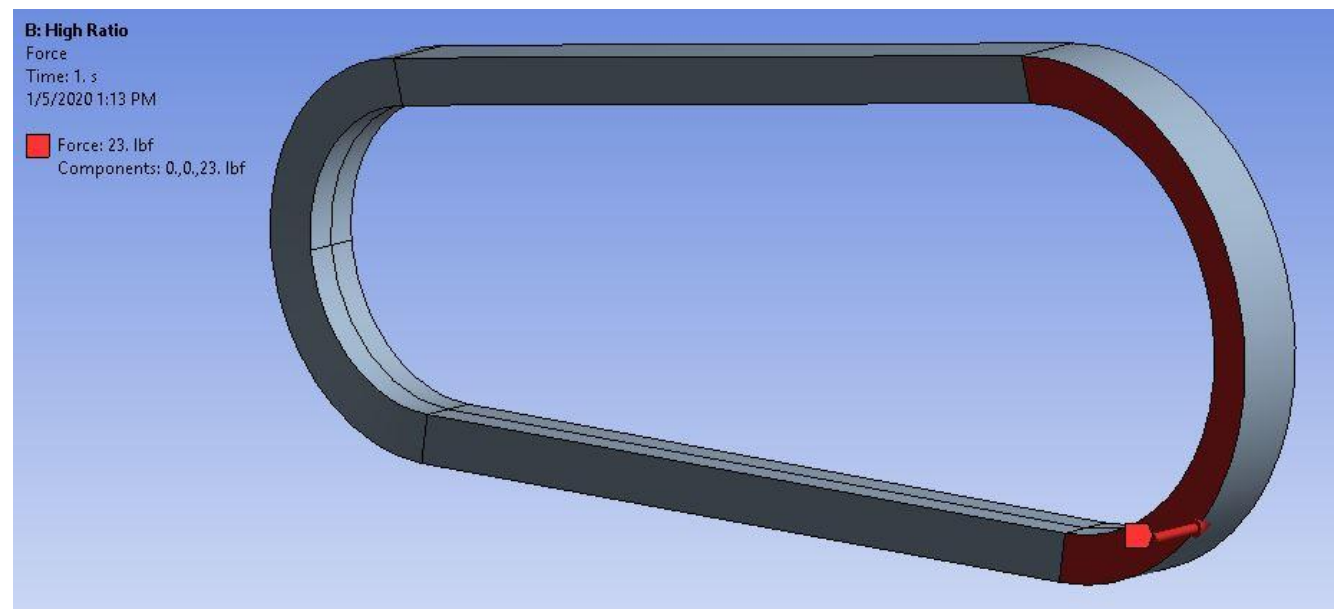

Figure 34: ANSYS Forces Applied on Belt Model. 


\section{Testing:}

After modeling CVT performance in MATLAB and observing result reliability in ANSYS, the final step was real world testing. The primary characteristic observed is the RPM of the motor during shift out. Results from testing were compared to the predictions from MATLAB. A secondary objective was to determine engine horsepower by testing without the CVT. This would be compared to the manufactures defined $10 \mathrm{hp}$ for the M19 engine. To meet these objectives, testing was performed with a water-brake dynamometer and Arduino based data acquisition (DAQ) setup.

\section{Water-brake Dynamometer}

The dynamometer used is a land and Sea water-brake dynamometer. This style of dyno works like a centrifugal water pump. It contains several vaned rotors that can be spun up by spinning the shaft it is mounted on. These vanes have cupped sections that push water through the dyno. Load is adjusted by controlling the volume of water circulating through the brake. Increasing the water level raises the total work required to spin the brake. A large portion of the work energy from the motor is converted into heat in the water. This heat must be dissipated before the water can be recirculated into the brake. This is done utilizing a simple heat exchanger setup with cold water flowing through. The water line passing through the brake is a closed loop to keep the water clean and free of impurities. This system utilizes a strain gauge to precisely measure the torque that is being exerted to move the water. Using the torque reading and the angular velocity the brake is spinning at, horsepower production at the brake is measured.

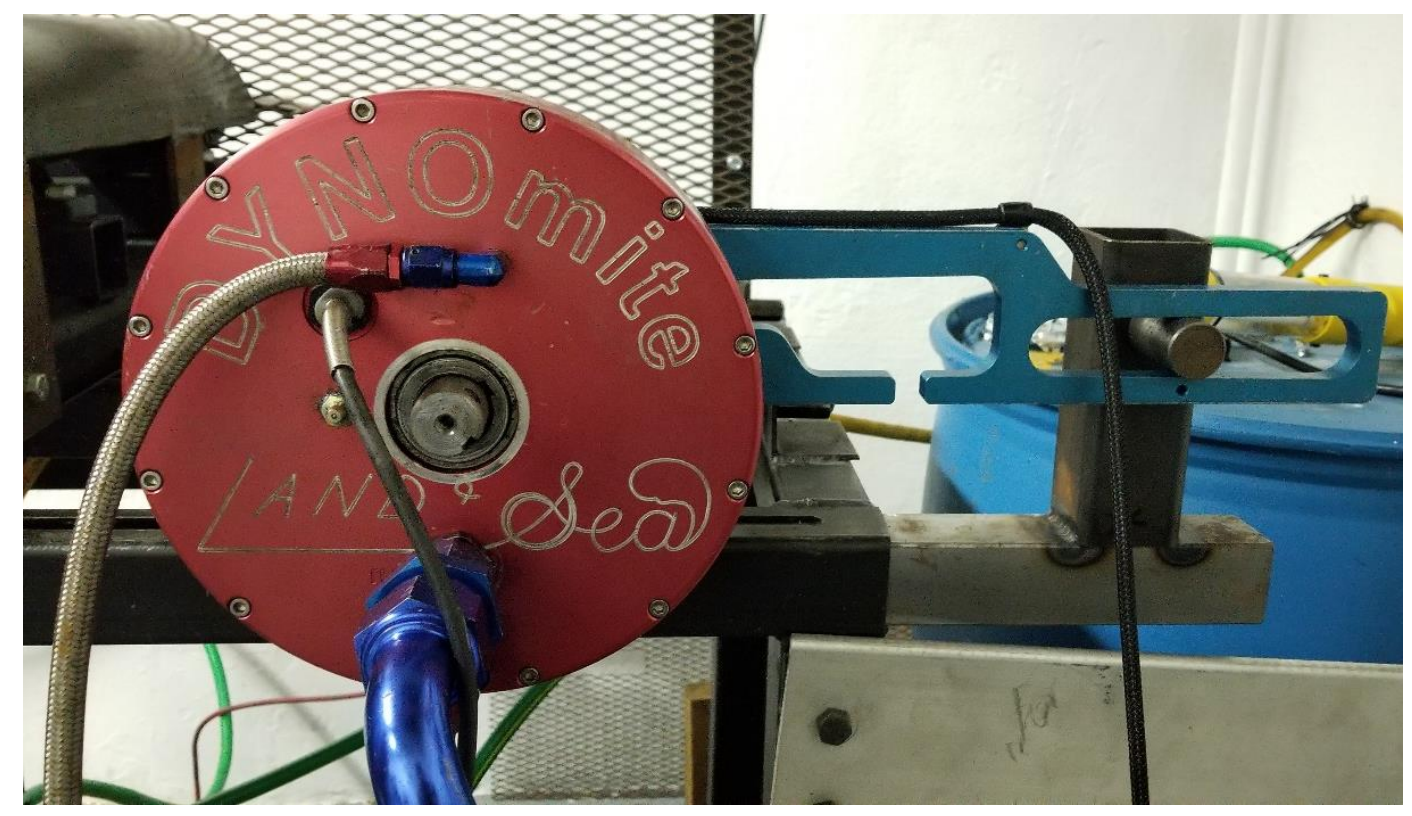

Figure 35: Land and Sea Water-brake Dynamometer on Test Stand.

Preparing to use the dyno requires designing a dyno table. The table allows the user to mount the engine, CVT, and dyno. There were several factors influencing the design of this table. Primarily, the dynamometer available for use was designed to be most accurate in a specific RPM 
range. For this dyno that range is between 4000-16,000 RPM. To ensure this RPM range could be met, the table was designed with a double reduction pulley system between the CVT secondary and the dyno input. This allowed for flexibility in dialing in the RPM at the dyno by changing pulley ratios. A secondary design influence is that WVU uses a variety of CVTs with different belt lengths. To prepare for this, the engine was mounted onto a plate with studs in slots. Moving this plate within the slots further tensions the belt. This allows for precise changes in tension, with a range of several inches.

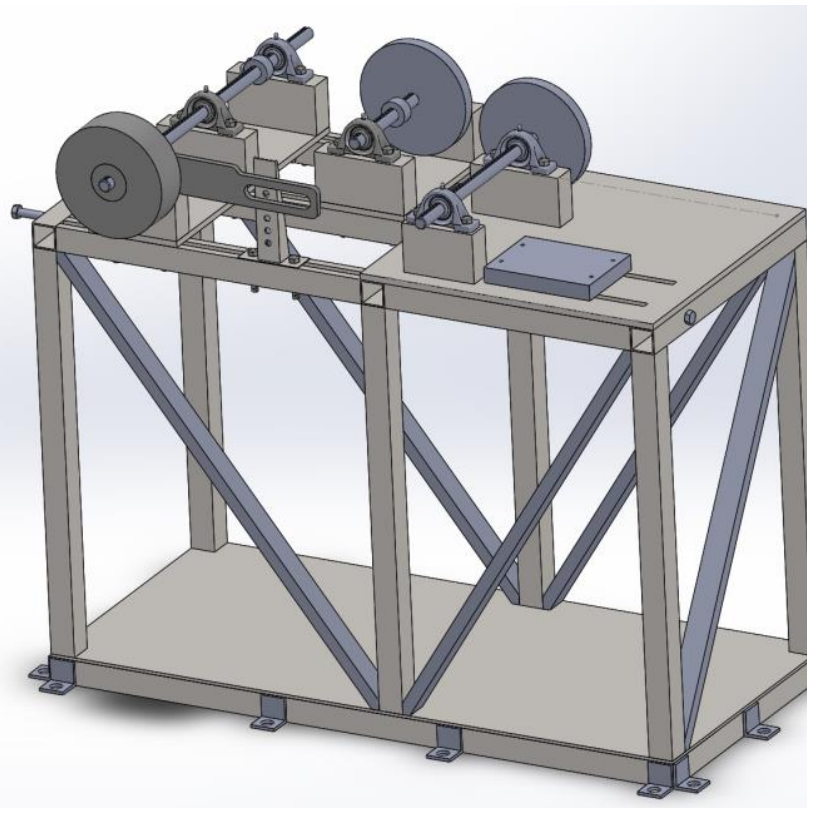

Figure 36: Dyno Table CAD Model.

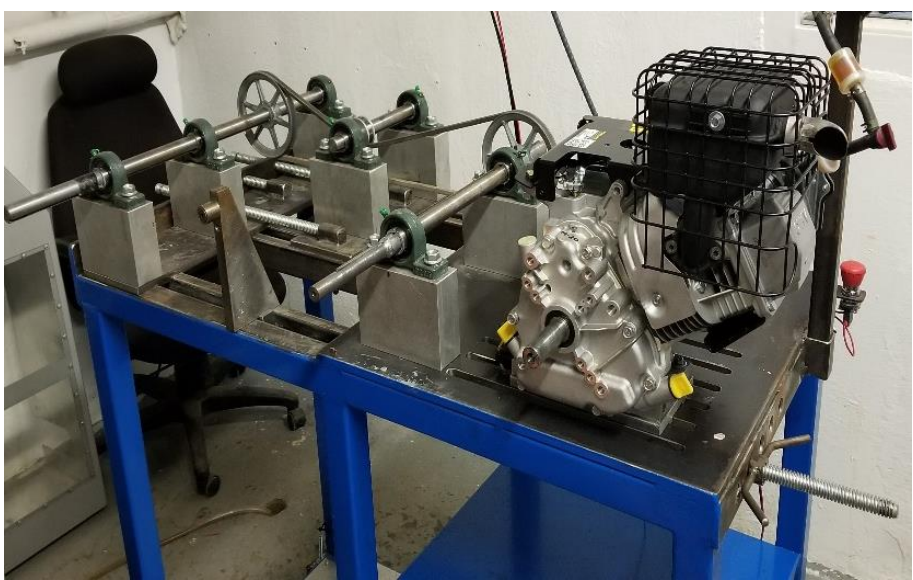

Figure 37: Dyno Table with Motor Mounted.

\section{Data Acquisition Setup:}

The DAQ system was used to monitor shaft speed of the primary and secondary. This system was created using an Arduino Mega with hall effect sensors and magnets mounted on the housing of the CVT. Hall effect sensors produce a voltage in the presence of a magnetic field. The output voltage varies proportionally to the strength of the field. Therefore, moving a magnet closer to the sensor will increase the voltage output. Several magnets were placed on the outside of the primary and secondary. The hall effect sensors were mounted so they would be able to detect the magnets go by as the motor spins up. The Arduino detects voltage rising and falling from the hall affect sensors. Once a falling voltage is detected, the Arduino counts the time until another magnet passes by. Using this information, the angular velocity of each shaft is calculated. The setup stores RPM of the primary and secondary against board time. This information is displayed on a small LCD screen in addition to be written to an SD card. 


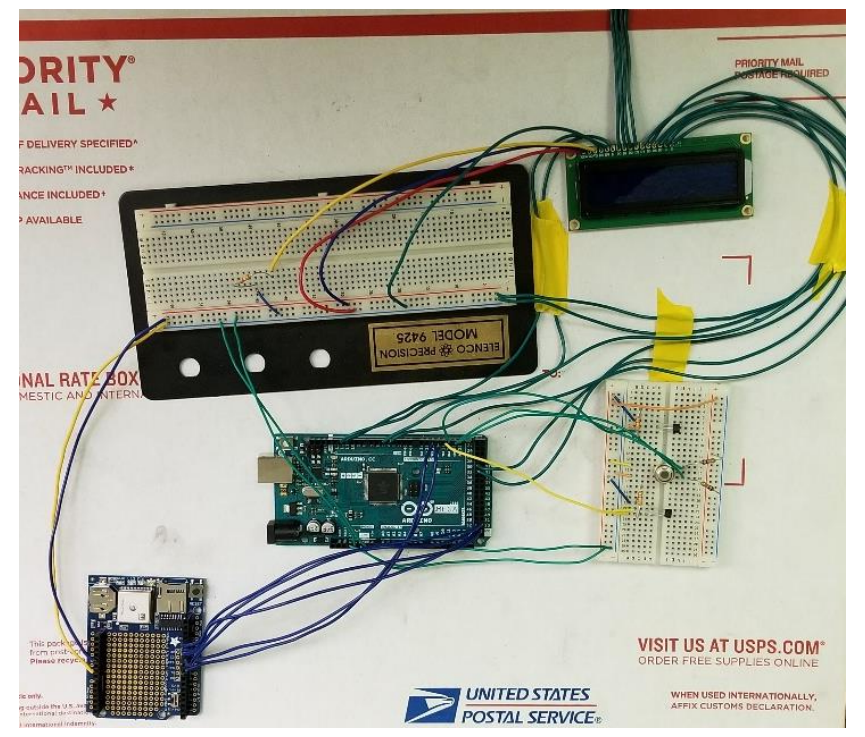

Figure 38: Arduino RPM Monitoring Setup. 


\section{Results:}

This section outlines results from testing, and all data found on CVT components. CVT data includes the constants needed for calculation, and the various options for system setups. Testing results include MATLAB calculations, FEA results from ANSYS, and speed graphs from testing.

\section{System Constants:}

Several of the system constants could simply be measured in house or taken from a credible online source. For most of the constants taken from an online source, testing was done to confirm their value. Table 1 lists several system constants measured in house. These include values such as sheave groove angle, gearbox ratio, initial spring compression, etc.

Table 2 outlines constants for the motor. These values were found on Briggs and Stratton's information page for the M19 engine. A dyno graph for the motor was provided on this page and was used to determine where peak motor torque and power occur. These graphs are displayed in Figure 39 and Figure 40. These values determined the desired engagement and shift RPM for an optimized setup. These graphs are displayed in Figure 39 and Figure 40.

Table 3 details properties of the Enduro 100 CVT belt. Geometric properties such as the pitch length and weight were measured in house. Material properties including the Young's modulus, poisons ratio, and nominal coefficient of friction were found from online sources.

Table 1: System Constants.

\begin{tabular}{|c|c|c|c|}
\hline \multicolumn{2}{|c|}{} & Value & Units \\
\hline \multirow{4}{*}{ System } & Gear Box Ratio & 7 & - \\
\cline { 2 - 4 } & Tire Radius & 11 & Inches \\
\hline \multirow{4}{*}{ Primary Sheaves } & Sheave Groove & 23 & Degrees \\
\cline { 2 - 4 } & Shift Displacement & 0.8 & Inches \\
\cline { 2 - 4 } & Initial Pitch Radius & 0.975 & Inches \\
\cline { 2 - 4 } & Full Shift Pitch Radius & 2.775 & Inches \\
\hline Pressure Spring & Initial Radius & 1.875 & Inches \\
\hline \multirow{4}{*}{ Secondary Sheaves } & Full Shift Radius & 2.345 & Inches \\
\cline { 2 - 4 } & Arm Mass (Each) & 0.001 & Sluggs \\
\cline { 2 - 4 } & Shealled Displacement & 0.975 & Inches \\
\cline { 2 - 4 } & Shift Displacement & 23 & Degrees \\
\cline { 2 - 4 } & Full Shift Pitch Radius & 2.498 & Inches \\
\hline Helix & Ramp Radius & 1.455 & Inches \\
\hline \multirow{2}{*}{ Torsional Spring } & Installed Displacement & 1.44 & Inches \\
\cline { 2 - 4 } & Full Shift Twist & 40 & Degrees \\
\hline
\end{tabular}


Table 2: Motor Constants.

\begin{tabular}{|c|c|c|c|c|}
\hline \multicolumn{2}{|c|}{} & Value & Units & Source \\
\hline \multirow{4}{*}{ Motor } & Engagement Power & 7 & Hp & \\
\cline { 2 - 4 } & Shift Power & 8.5 & Hp & \multirow{3}{*}{ Briggs and Stratton Racing } \\
\cline { 2 - 4 } & Desired Engagement RPM & 2800 & RPM & \\
\cline { 2 - 4 } & Desired Shift RPM & 3600 & RPM & \\
\hline
\end{tabular}

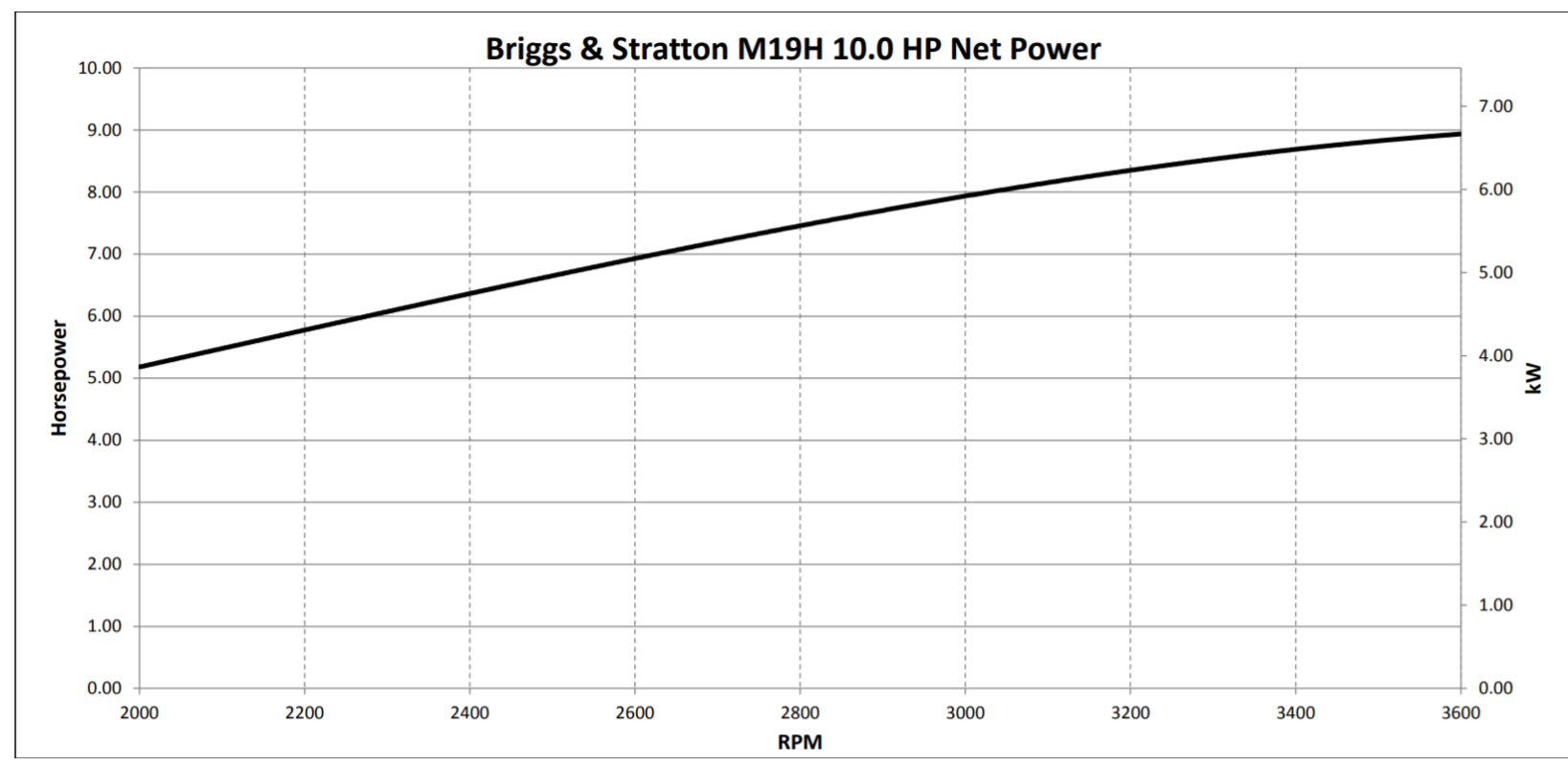

Figure 39: Briggs and Stratton M19 Engine Power Curve. 


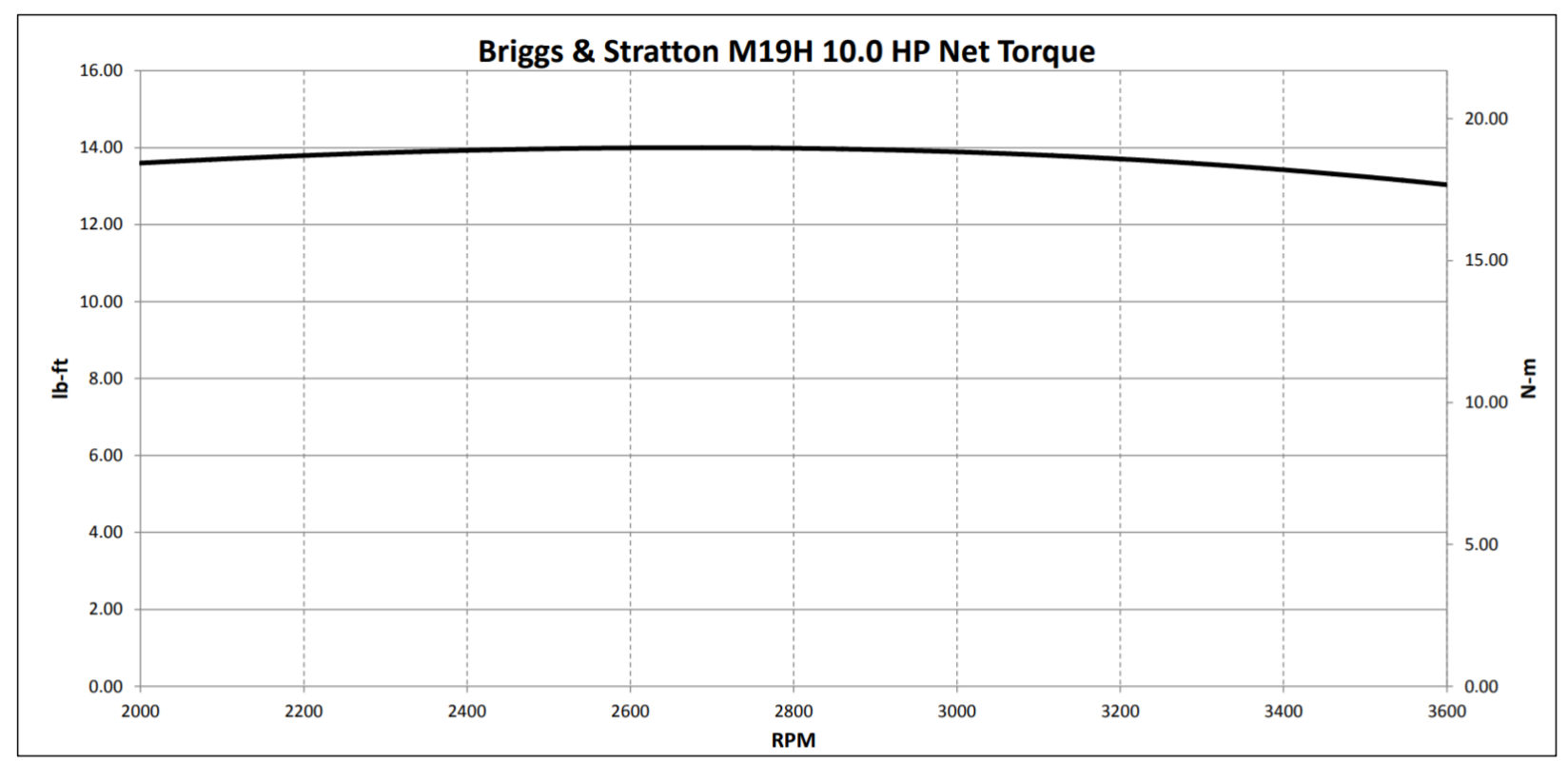

Figure 40: Briggs and Stratton M19 Engine Torque Curve.

Table 3: Belt Constants.

\begin{tabular}{|c|c|c|c|c|}
\hline & & Value & Units & Source \\
\hline \multirow{4}{*}{ Belt } & Cross Section Area & 0.41 & in.^2 & Measured \\
\cline { 2 - 5 } & Pitch Length & 38 & in. & Measured \\
\cline { 2 - 5 } & Weight & 0.6 & lbs & Measured \\
\cline { 2 - 5 } & Youngs Modulus & 13.8 & $\mathrm{ksi}$ & \multirow{2}{*}{ Messick } \\
\cline { 2 - 5 } & Shear Modulus & 5.08 & $\mathrm{ksi}$ & \\
\cline { 2 - 5 } & Center to Center & 8.5 & Inches & Gaged Engineering \\
\cline { 2 - 5 } & Nominal COF & 0.13 & - & Richard G. Budynas \\
\hline
\end{tabular}

\section{Spring Constants:}

Compression tests were conducted with the test rig for all springs. Resultant constants from these trials are listed below in Table 4. However, torsional tests did not go as smoothly. When GX9 springs are twisted they must also be compressed, due to the spaces between their wraps. This means that while turning the spring with the torque wrench, the user also needs to press down on the spring to allow it to continue twisting. When in use, the CVT is already compressing the spring as it is twisted, so this is not a problem. This detail made accurate torsional testing with the spring rig difficult, and reduced precision. The torque wrench wasn't providing any response until approximately 45 degrees, and torque response wasn't stable until around 135 degrees twisted. Due to these inconsistencies, torsional constants calculated from Equation 3 were used for analysis and are also listed in Table 4. Data for all trials conducted are listed in the appendix. 
Figure 41 and Figure 42 show plots of linear spring force vs displacement. This plot was created to answer the question: can these springs be treated as linear springs? Does the spring force increase linearly through full compression? The plots show that in general the spring constants were not completely consistent throughout compression. To mitigate this, these data points were fit onto a line using the least squares method. The slope of this line was then the resultant spring constant used and recorded in Table 4.

Table 4: Spring Constants.

\begin{tabular}{|c|c|c|c|}
\hline \multicolumn{1}{|c|}{} & Linear (lbf/In) & \multirow{2}{*}{ Torsional (In-lbf/Deg) } \\
\hline \multirow{4}{*}{ Pressure Spring } & Old Black & 42.71 & \multirow{2}{*}{} \\
\cline { 2 - 3 } & New Black & 43.75 & \\
\cline { 2 - 3 } & New Orange & 84.79 & \\
\cline { 2 - 3 } & New Purple & 79.89 & \\
\hline \multirow{4}{*}{ Helical Spring } & Old Yellow & 21.25 & \\
\cline { 2 - 3 } & Old Red & 25.23 & 0.4644 \\
\cline { 2 - 3 } & New Yellow & 22.75 & 0.5920 \\
\cline { 2 - 3 } & New Red & 14.65 & 0.4644 \\
\hline
\end{tabular}

Pressure Spring: Linear Spring Force Vs. Displacement

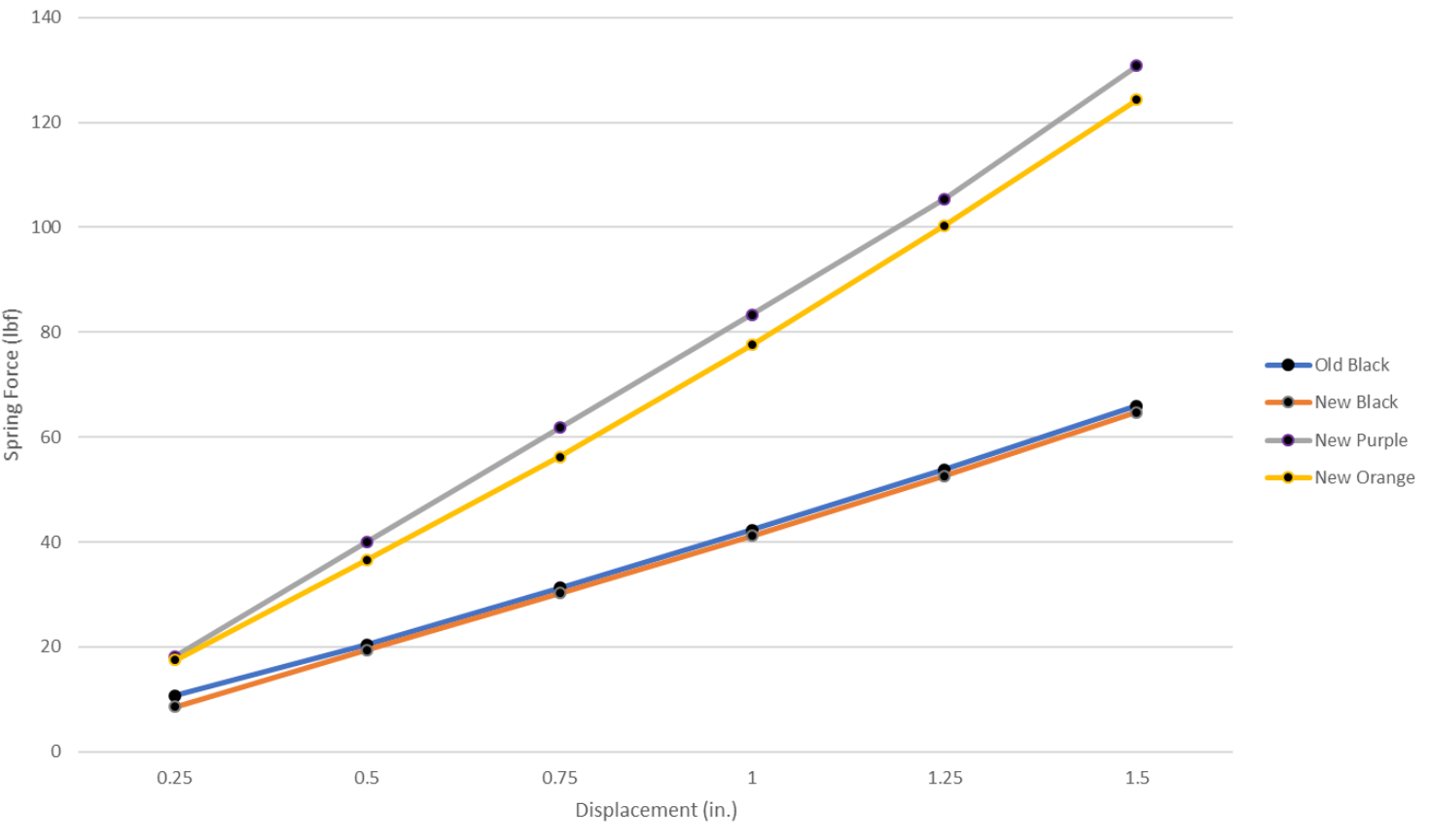

Figure 41: Pressure Spring: Linear Spring Force Vs. Displacement. 


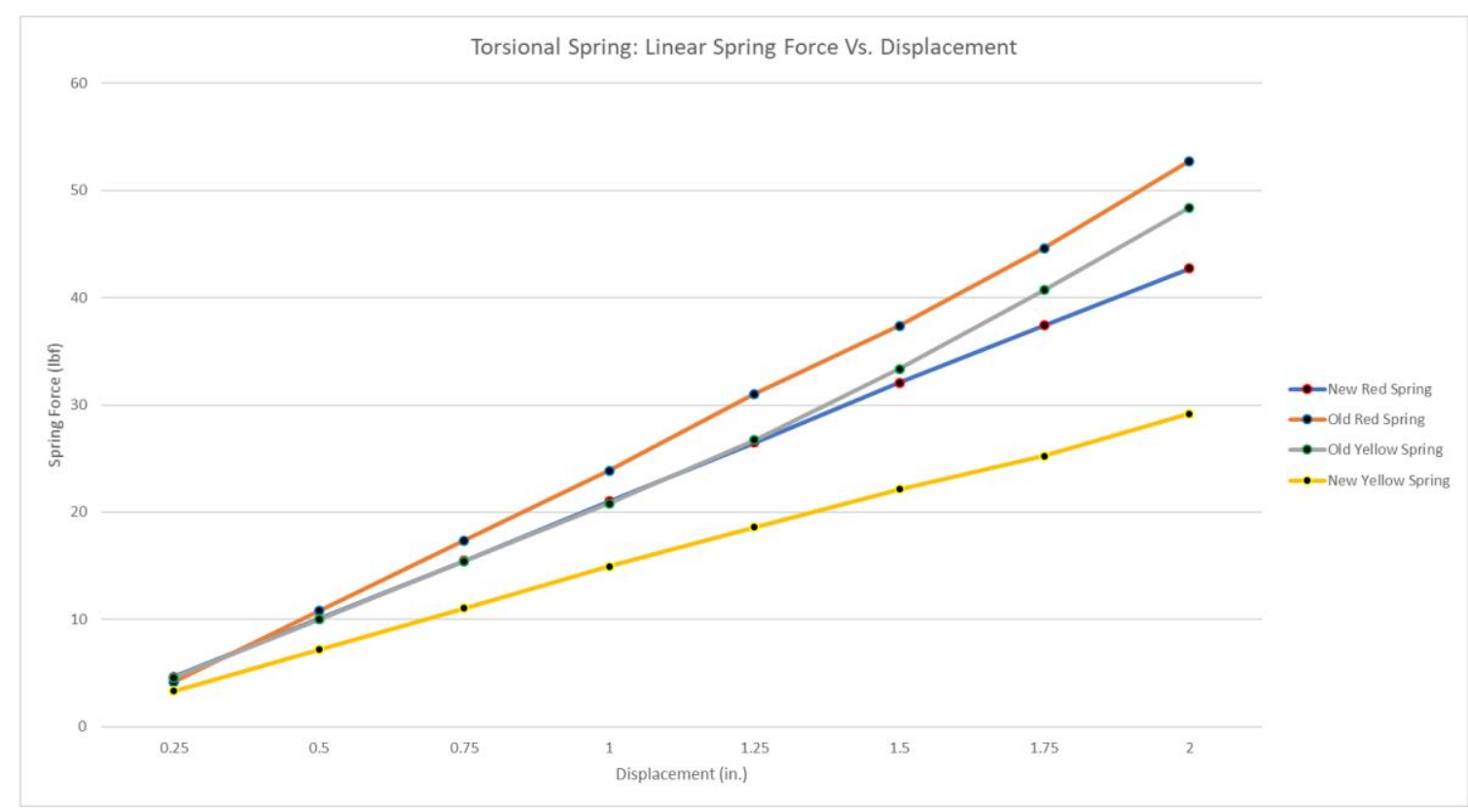

Figure 42: Torsional Spring: Linear Spring Force Vs. Displacement.

\section{Tunable Component Options:}

Table 5 lists all options for the systems tunable components. This table was referenced when naming different CVT setups. Each setup was assigned a 7-digit number where each digit is the "Option" selected for each variable. The order for each variable follows the descending order of Table 5 .

Table 5: Tunable Component Options.

\begin{tabular}{|c|c|c|c|c|c|c|c|c|}
\hline System & Variable & Units & \multicolumn{7}{|c|}{ Options } \\
\hline Pressure Spring & Linear Rate & In.-lbf & 42.71 & 43.75 & 79.89 & 84.79 & & \\
\hline \multirow{2}{*}{ Flyweight System } & Ramp Angle & Degrees & $10-13$ & $10-16$ & $14-17$ & 20 & & \\
\cline { 2 - 11 } & Mass (Per Arm) & Sluggs & 0.141 & 0.192 & 0.214 & 0.243 & 0.265 & 0.287 \\
\hline \multirow{3}{*}{ Torsional Spring } & Linear Rate & In.-lbf & 14.65 & 21.25 & 22.75 & 25.23 & & \\
\cline { 2 - 10 } & Pretension & Degrees & 45 & 46 & 67 & 78 & 89 & 100 \\
\cline { 2 - 10 } & Torsional Rate & In.-lbf/Deg & 0.464 & 0.595 & & & & \\
\hline Helix & Ramp Angle & Degrees & 25 & $24-32$ & 36 & 40 & 45 & \\
\hline
\end{tabular}

\section{MATLAB Calculations:}

Calculations in MATLAB looped through every setup combination outlined in Table 5. Utilizing all options yielded a total 11,520 different possible setups. Several design criteria were 
put in place to truncate setup options if they didn't meet specified criteria. Results in this section required a shift speed between 3400 and 3800 RPM, maximum transferrable torque at the primary and secondary must not fall below $80 \%$ of the minimum requirement, and the engagement speed must be above 2100 RPM. Stricter requirements were placed on the shift speed because it was considered the primary objective for this tuning. These requirements can easily be adjusted if specific areas of performance are deemed more important. On the following graphs, each line represents the performance of a different setup combination. There were 84 setups found that stayed within the design objectives. Finally, for these tests the vehicle weight was set at $350 \mathrm{lbs}$. and the hill angle was set at 20 degrees above horizontal.

Figure 43 and Figure 44 show the engine speed vs vehicle speed and shift ratio. Figure 45 is the vehicle speed vs the shift ratio. Figure 46 and Figure 47 detail the clamping force from the sheaves at the primary and secondary while shifting. Figure 48 and Figure 49 show the total transferrable torque at the primary and secondary during the shift. Figure 50 and Figure 51 display the tension on either end of the belt at the primary and secondary during shift out.

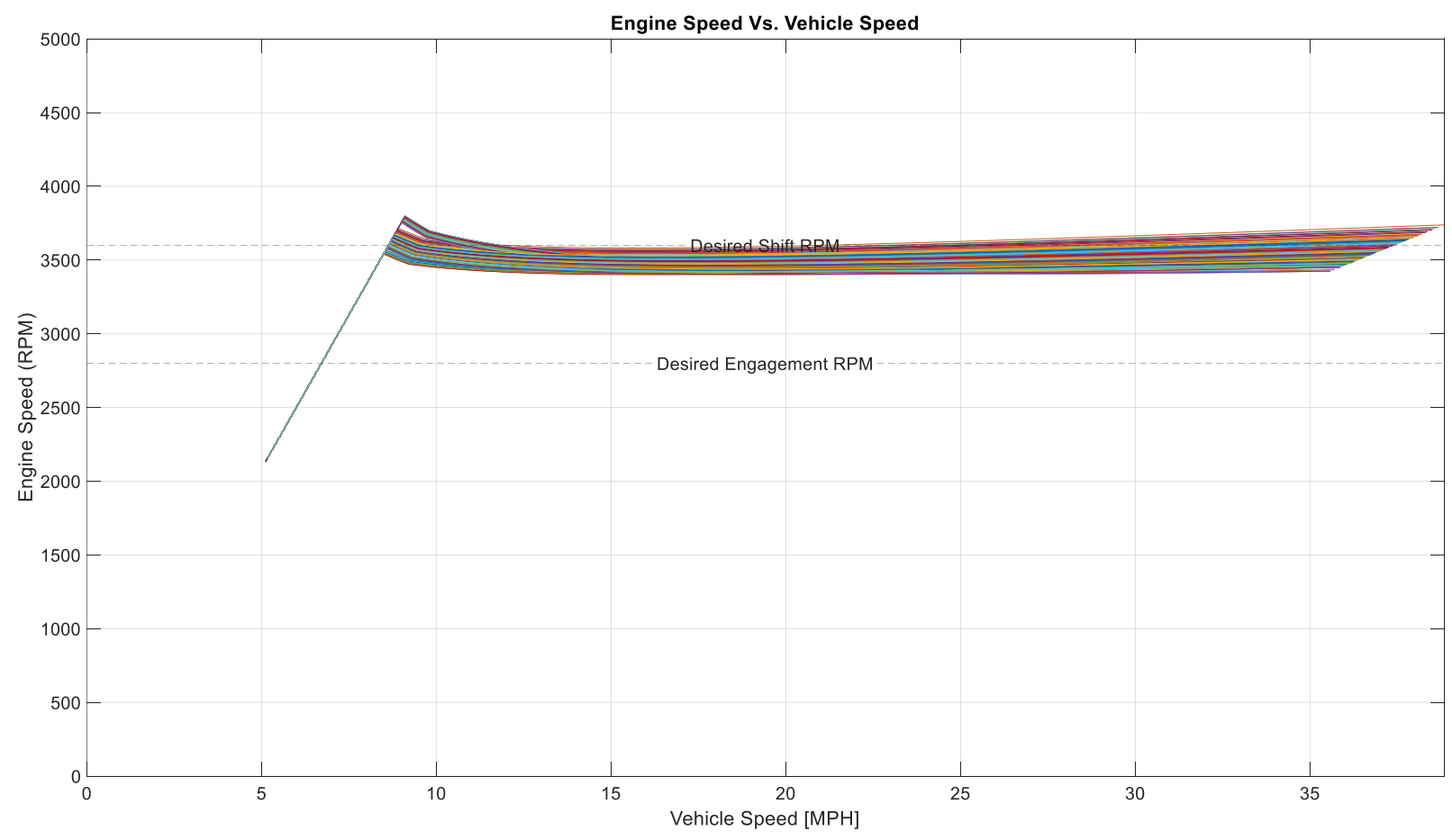

Figure 43: Engine Speed Vs. Vehicle Speed. 


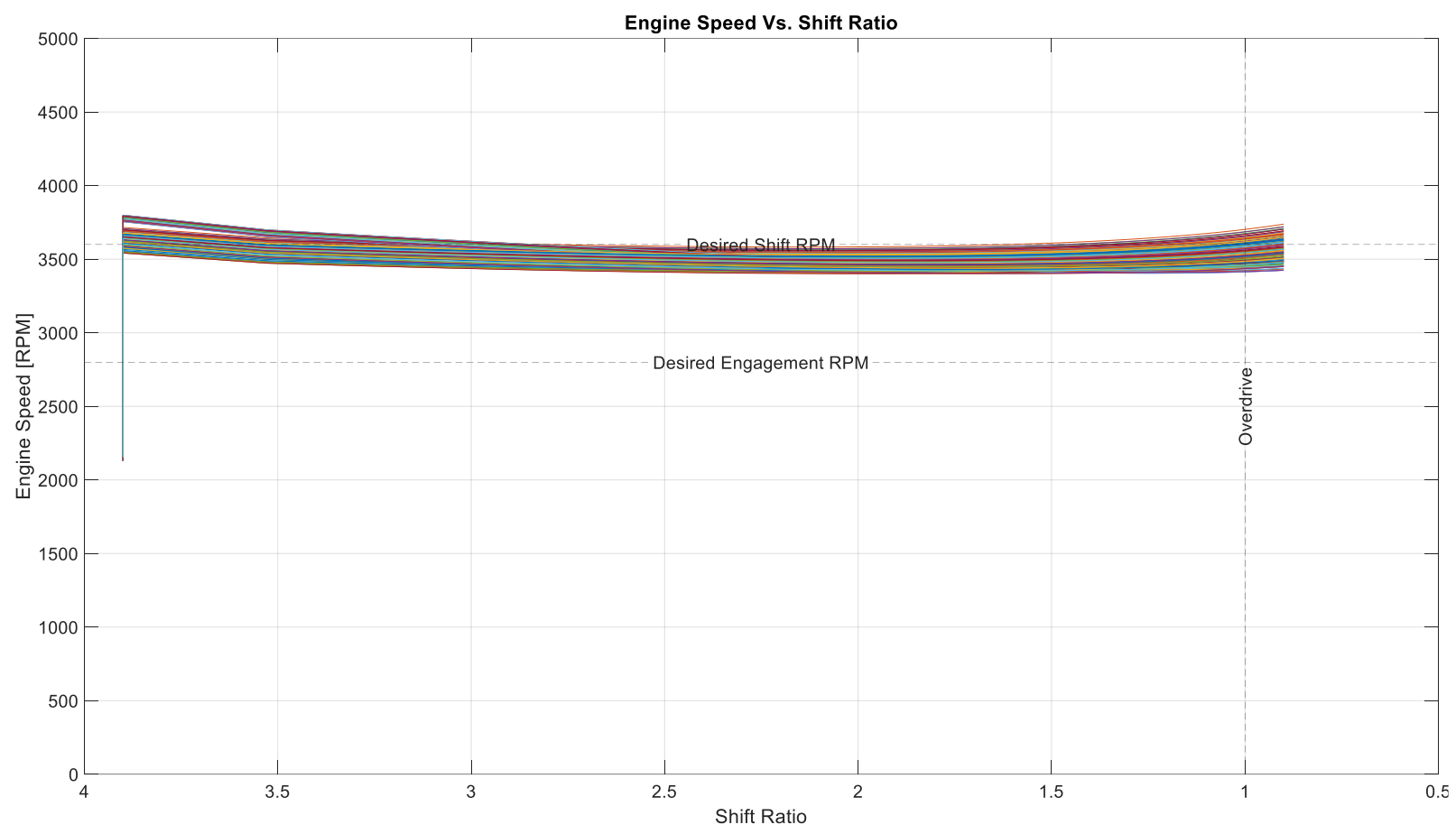

Figure 44: Engine Speed Vs. Shift Ratio.

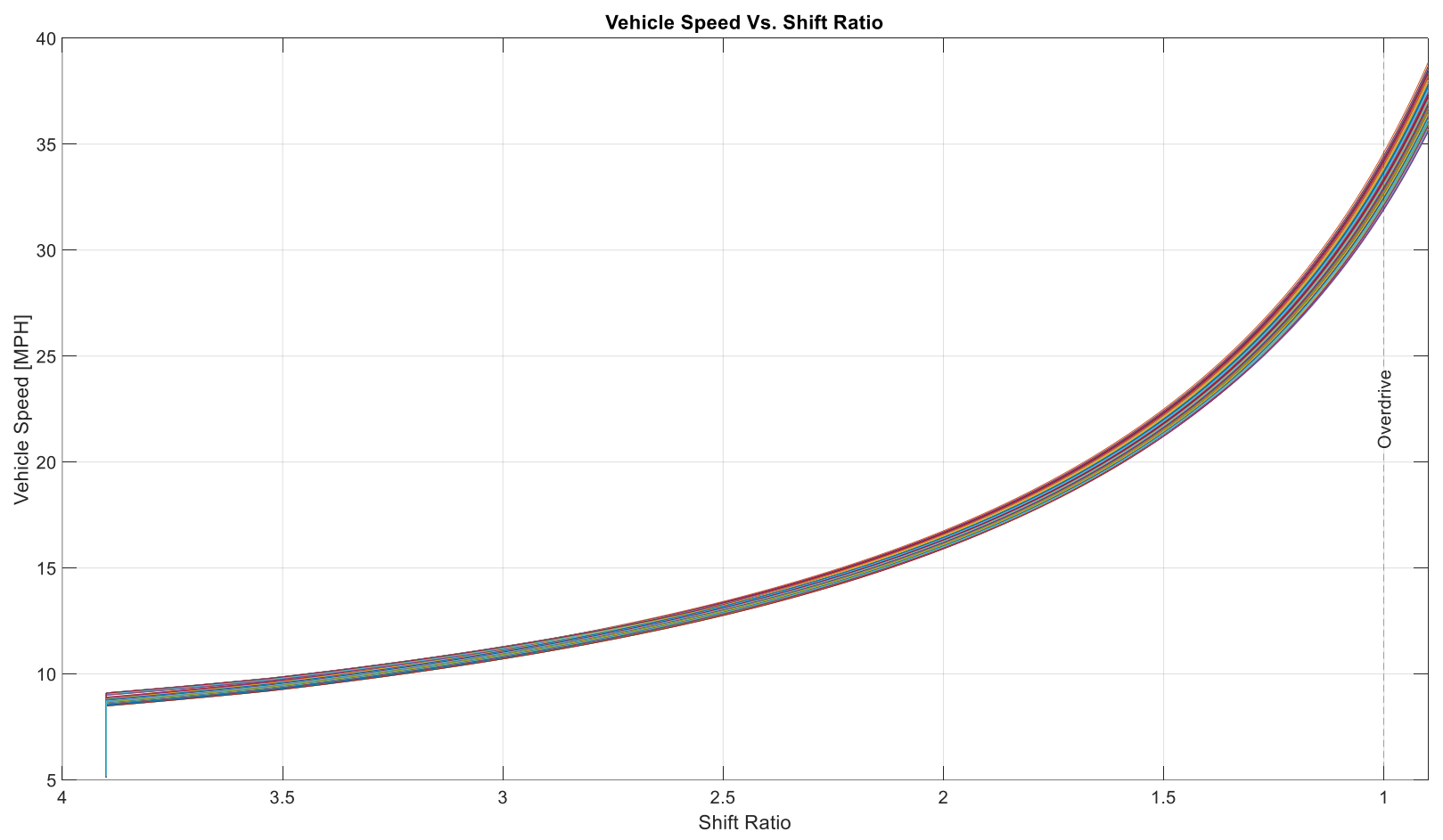

Figure 45: Vehicle Speed Vs. Shift Ratio, 


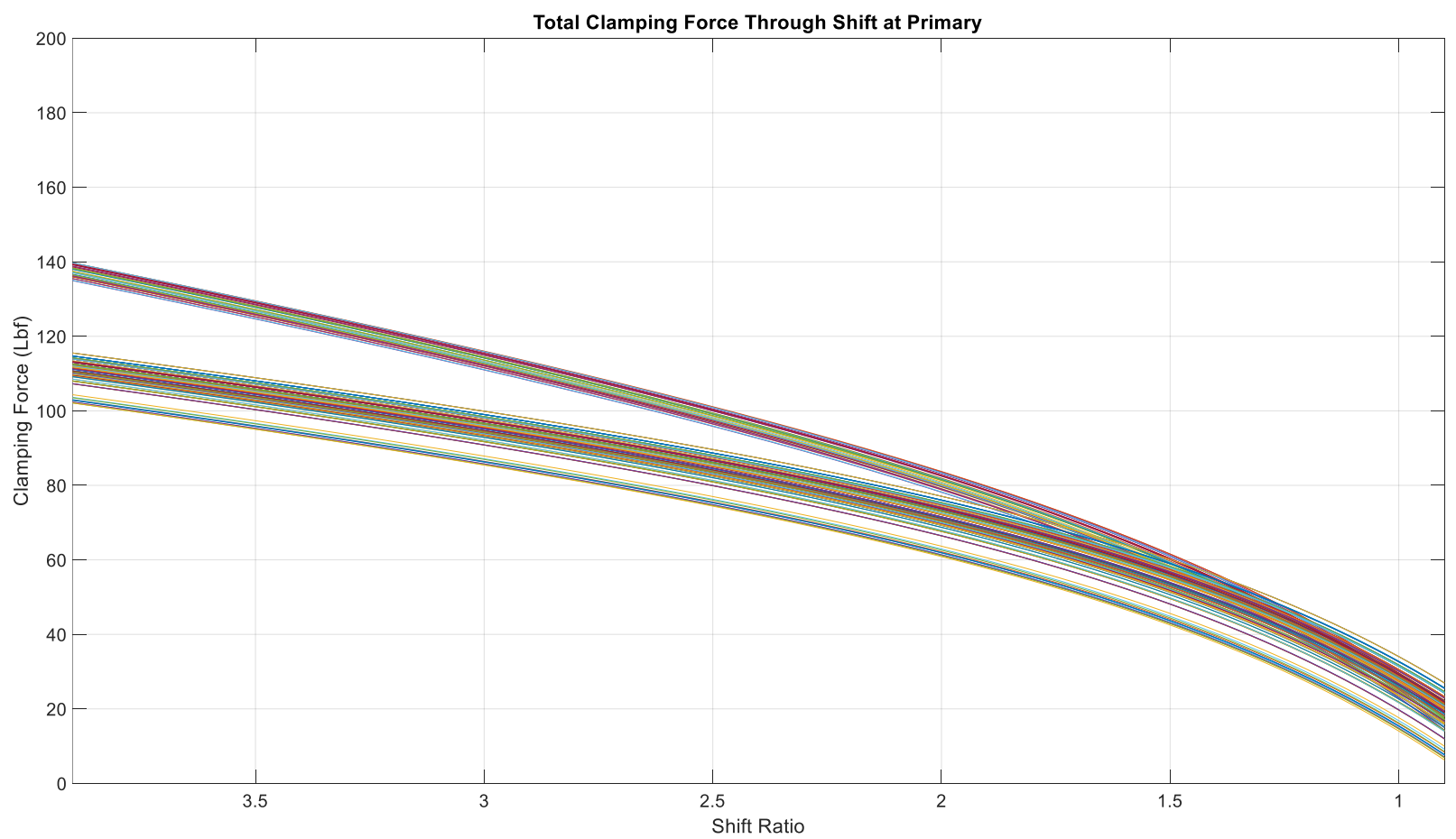

Figure 46: Clamping Force Through Shift at Primary.

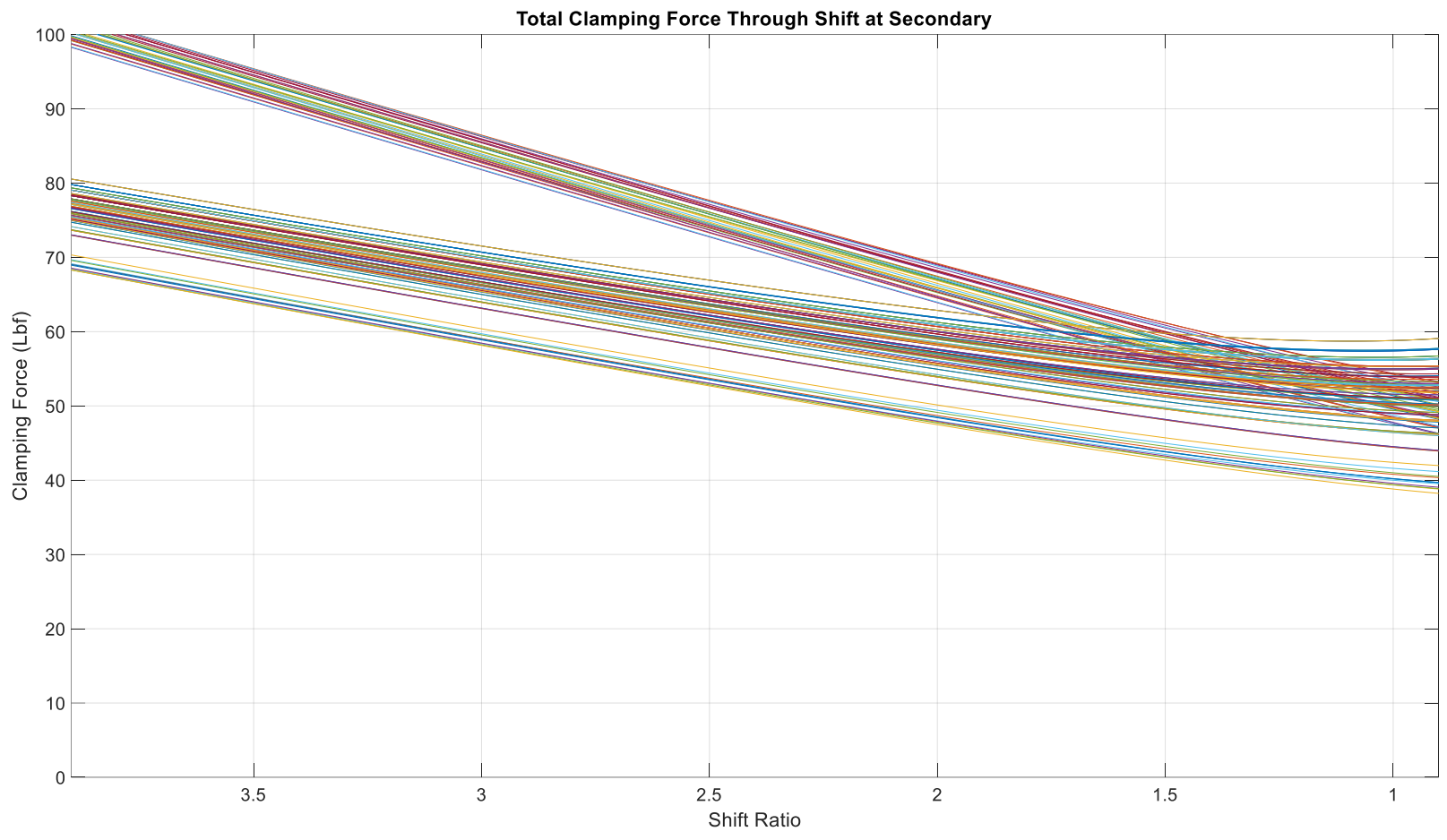

Figure 47: Clamping Force Through Shift at Secondary. 


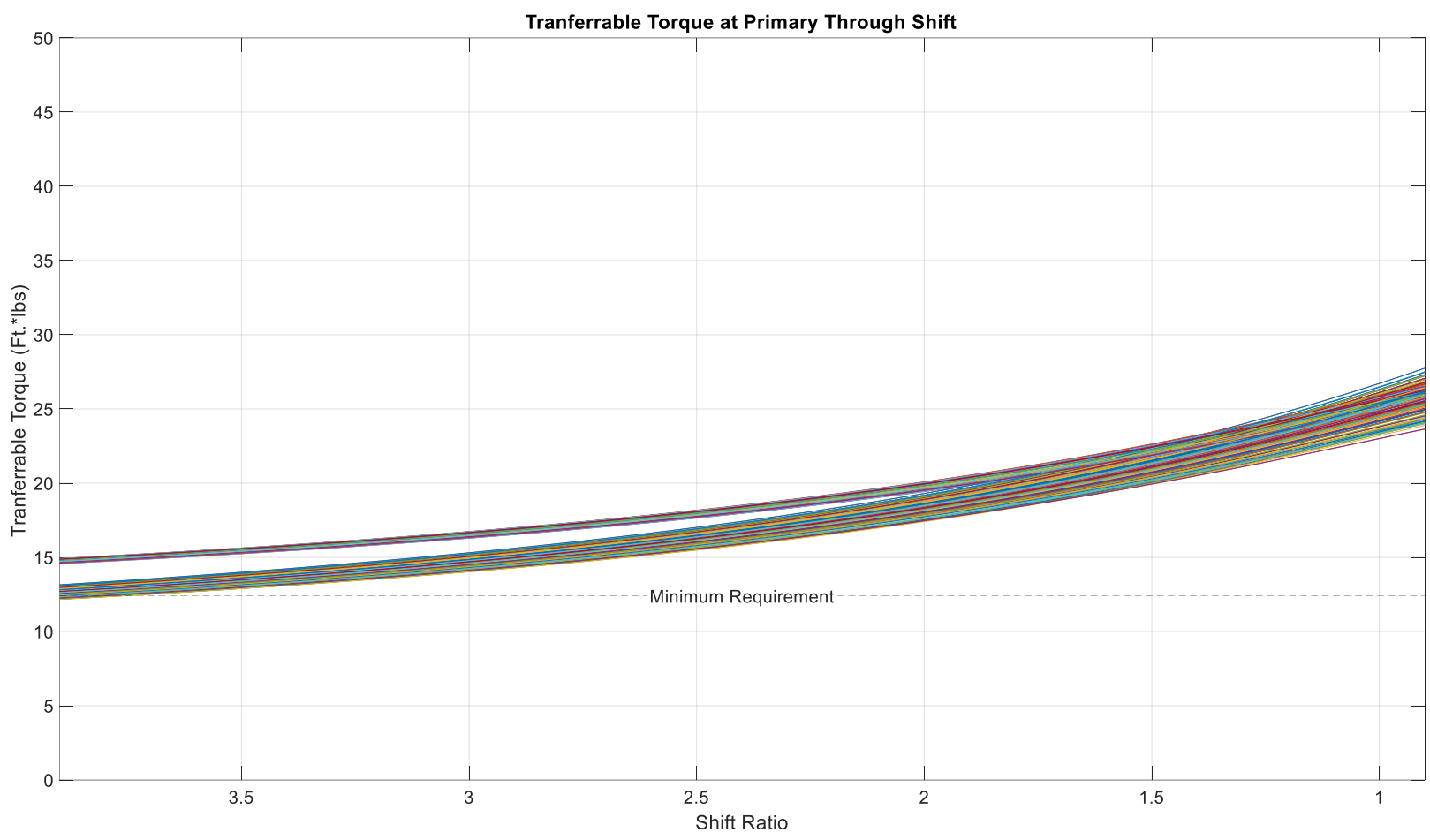

Figure 48: Transferrable Torque through shift at Primary.

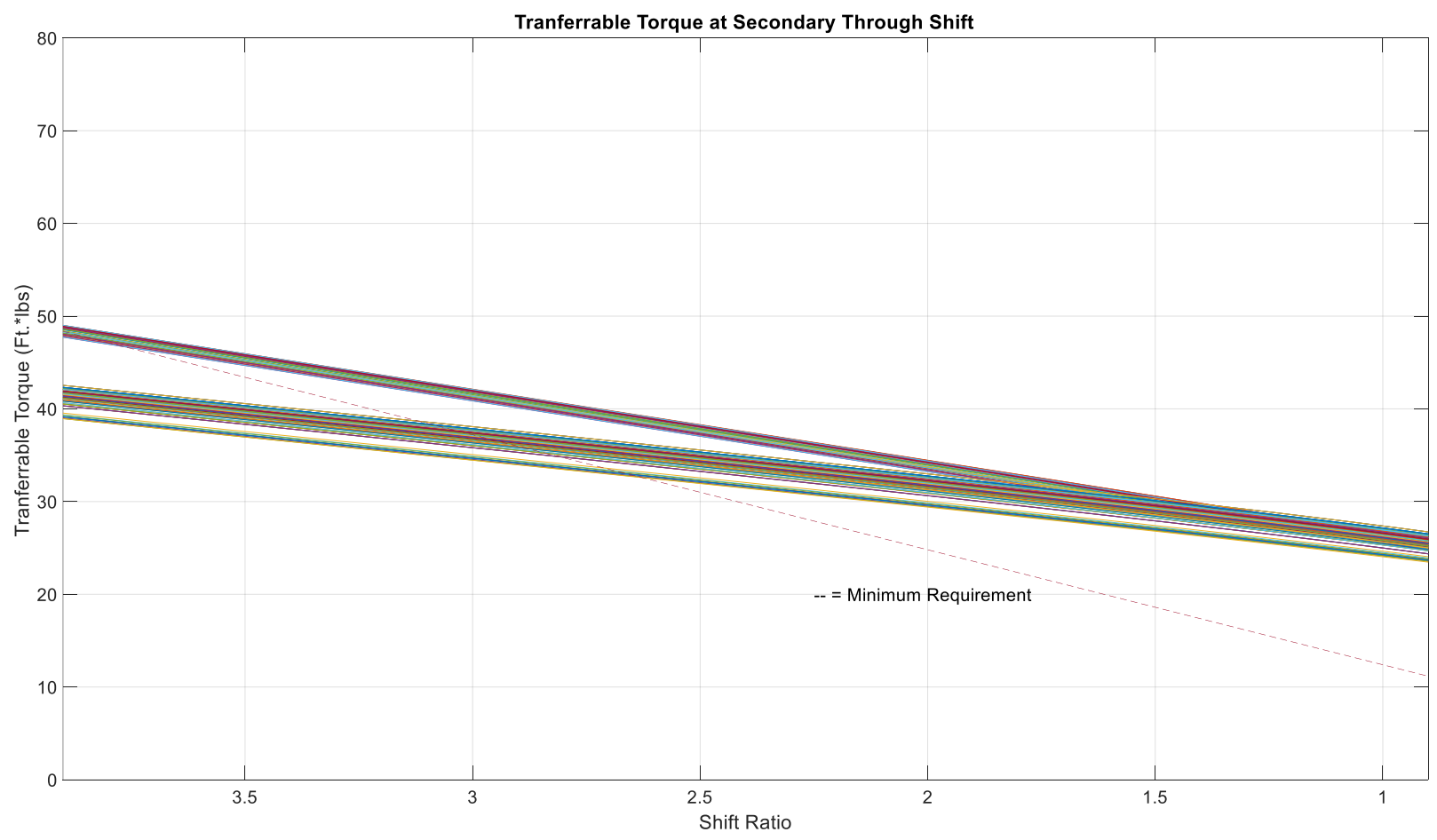

Figure 49: Transferrable Torque through shift at Secondary. 


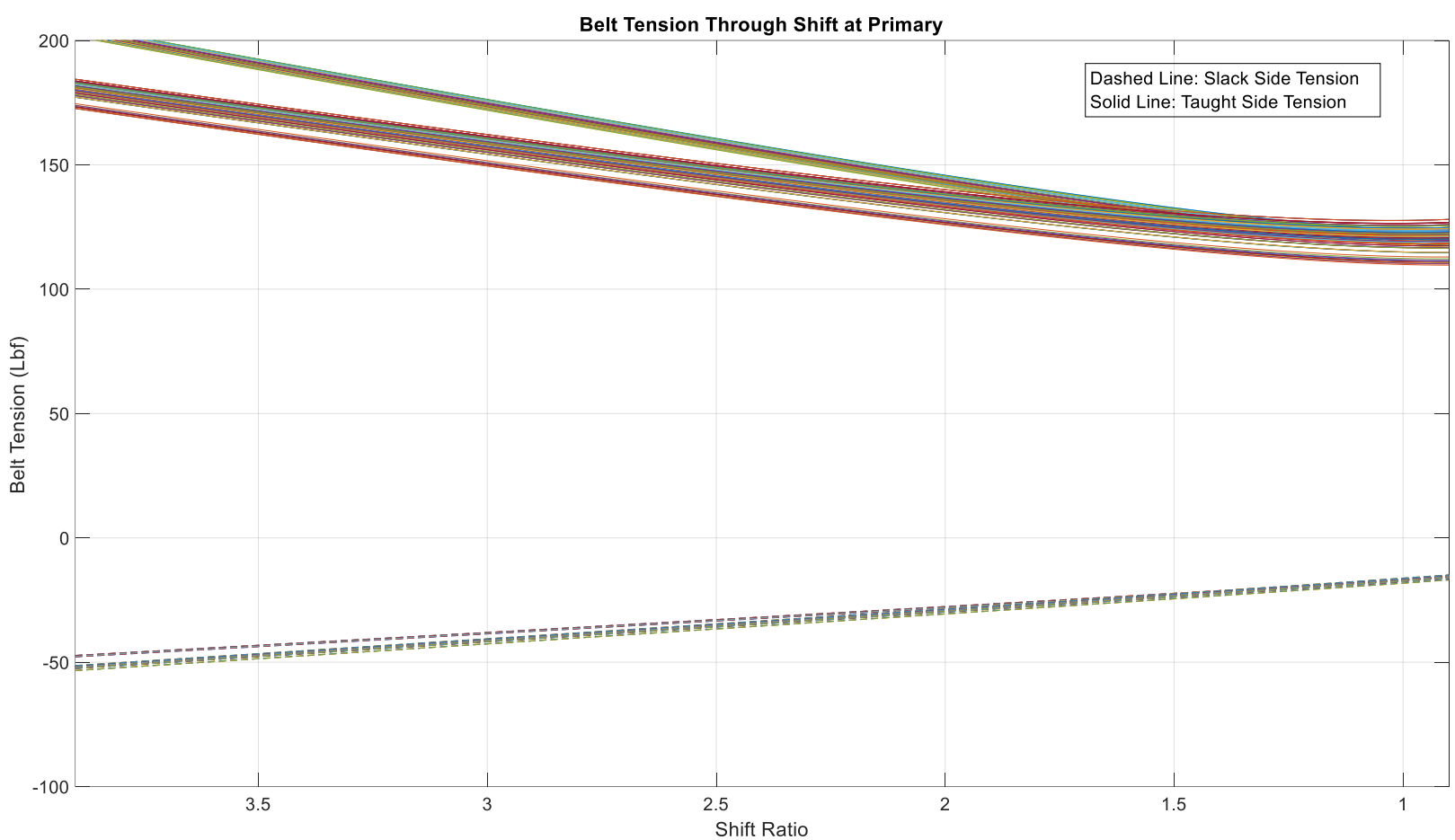

Figure 50: Belt Tension through shift at Primary.

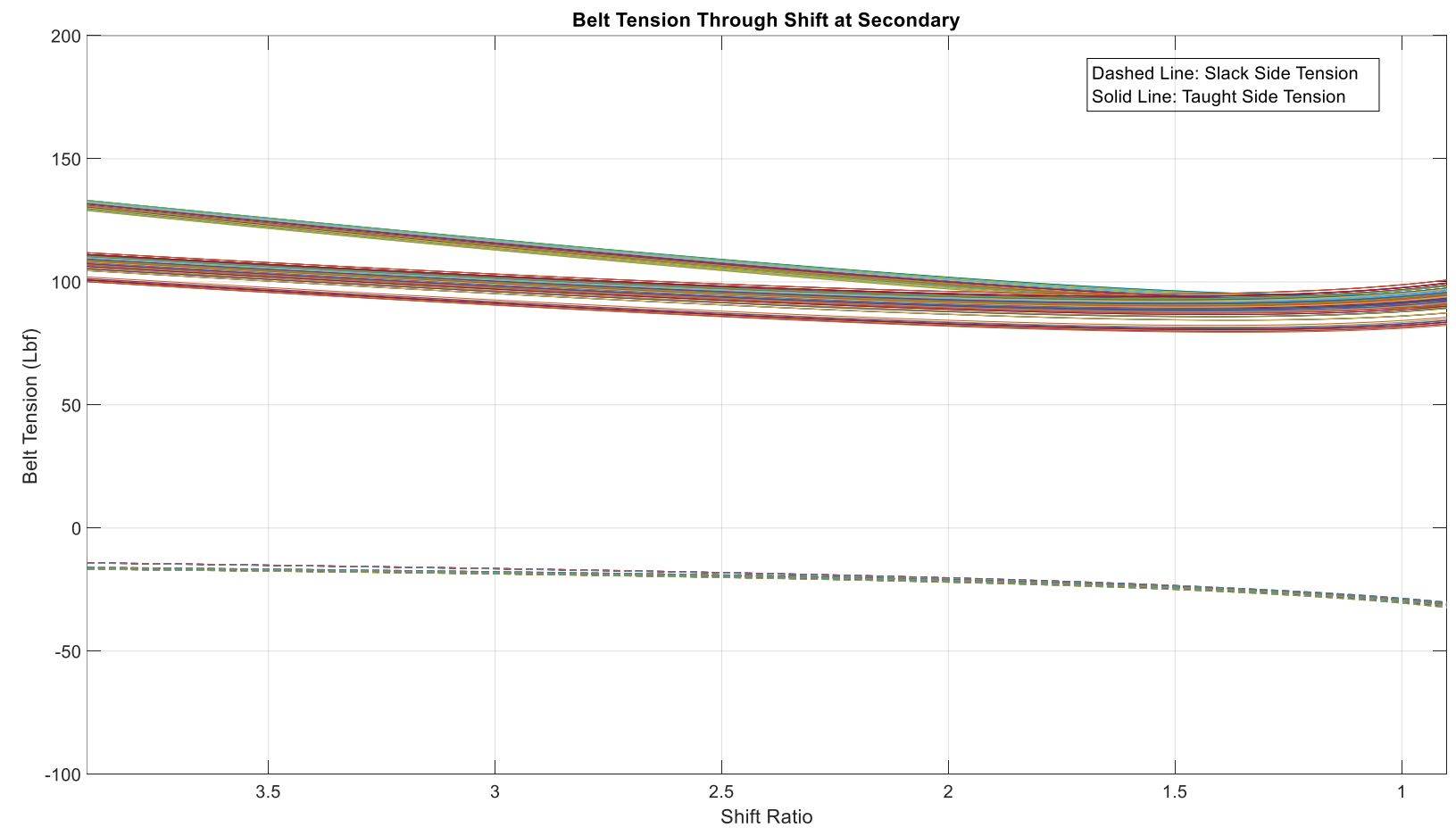

Figure 51: Belt Tension Through Shift at Secondary. 


\section{ANSYS Workbench Model:}

Stress results, and the associated tensions, are listed in Table 6. These results compare the tension calculated through FEA to those calculated in MATLAB and the error was calculated. Tests were conducted in low and high ratio, which changes the clamping force applied and the area of the face they are applied on. The resultant stress distributions are shown in Figure 52 and Figure 53.

Table 6: ANSYS Workbench Results.

\begin{tabular}{|c|c|c|c|c|}
\hline \multirow{4}{*}{ Primary } & \multicolumn{2}{|c|}{ Low Ratio } & \multicolumn{2}{c|}{ High Ratio } \\
\hline \multirow{5}{*}{} & Area (in^2) & 2.79 & Area (in^2) & 7.85 \\
\cline { 2 - 5 } & Clamping Force (lbf) & 110.00 & Clamping Force (lbf) & 23.00 \\
\cline { 2 - 5 } & Stress (Ansys) & 50.00 & Stress (Ansys) & 5.50 \\
\cline { 2 - 5 } & Tension (Ansys) & 139.50 & Tension (Ansys) & 43.18 \\
\cline { 2 - 5 } & Tension (MATLAB) & 116.00 & Tension (MATLAB) & 66.00 \\
\cline { 2 - 5 } & \% Difference & -20.26 & \% Difference & 34.58 \\
\hline \multirow{4}{*}{ Secondary } & 11.56 & \multicolumn{2}{c}{ Area } & 5.54 \\
\cline { 2 - 5 } & Area & 75.00 & Clamping Force & 48.00 \\
\cline { 2 - 5 } & Clamping Force & 8.00 & Stress (Ansys) & 10.00 \\
\cline { 2 - 5 } & Tension (Ansys) & 92.48 & Tension (Ansys) & 55.40 \\
\cline { 2 - 5 } & Tension (MATLAB) & 63.00 & Tension (MATLAB) & 62.50 \\
\cline { 2 - 5 } & \% Difference & -31.88 & \% Difference & 12.82 \\
\hline
\end{tabular}




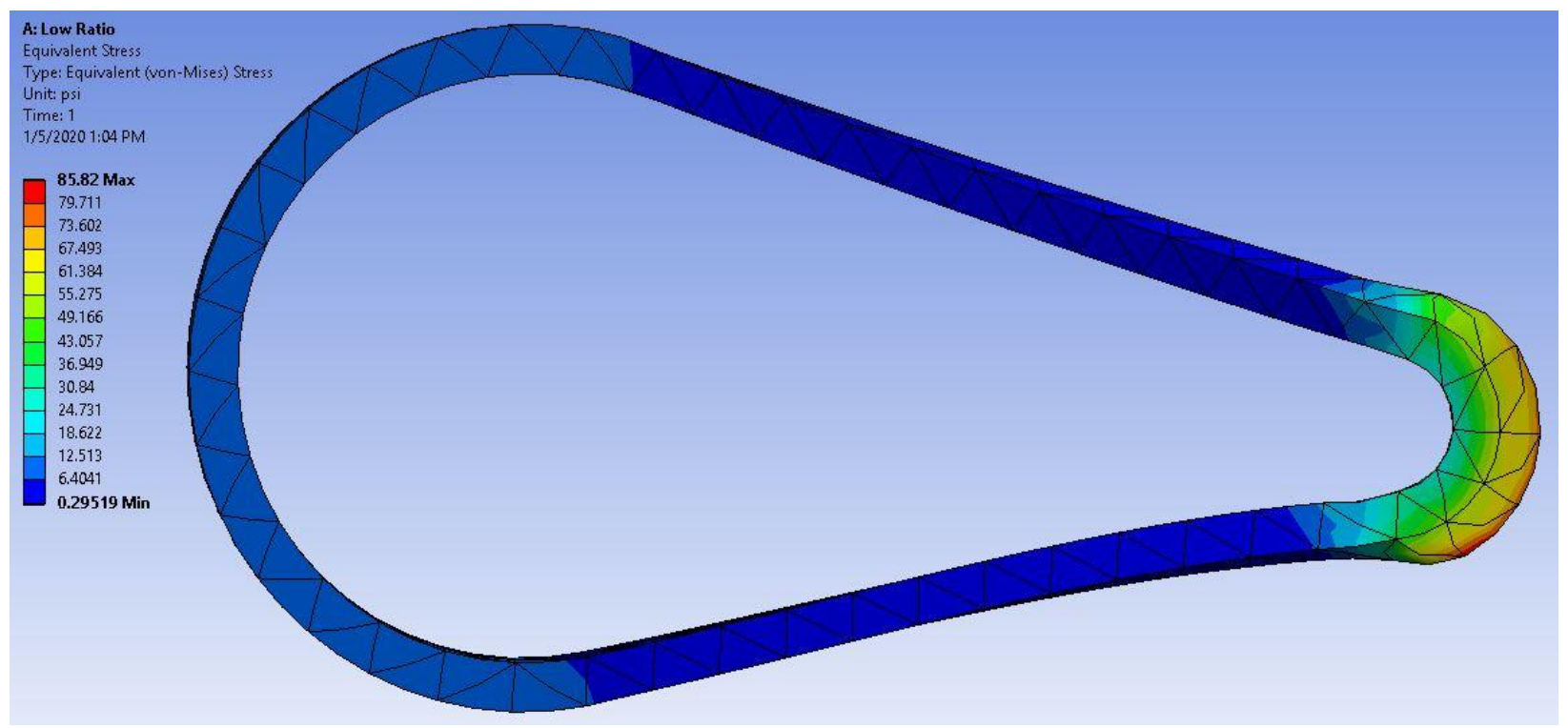

Figure 52: ANSYS Low Ratio Stress Distribution.

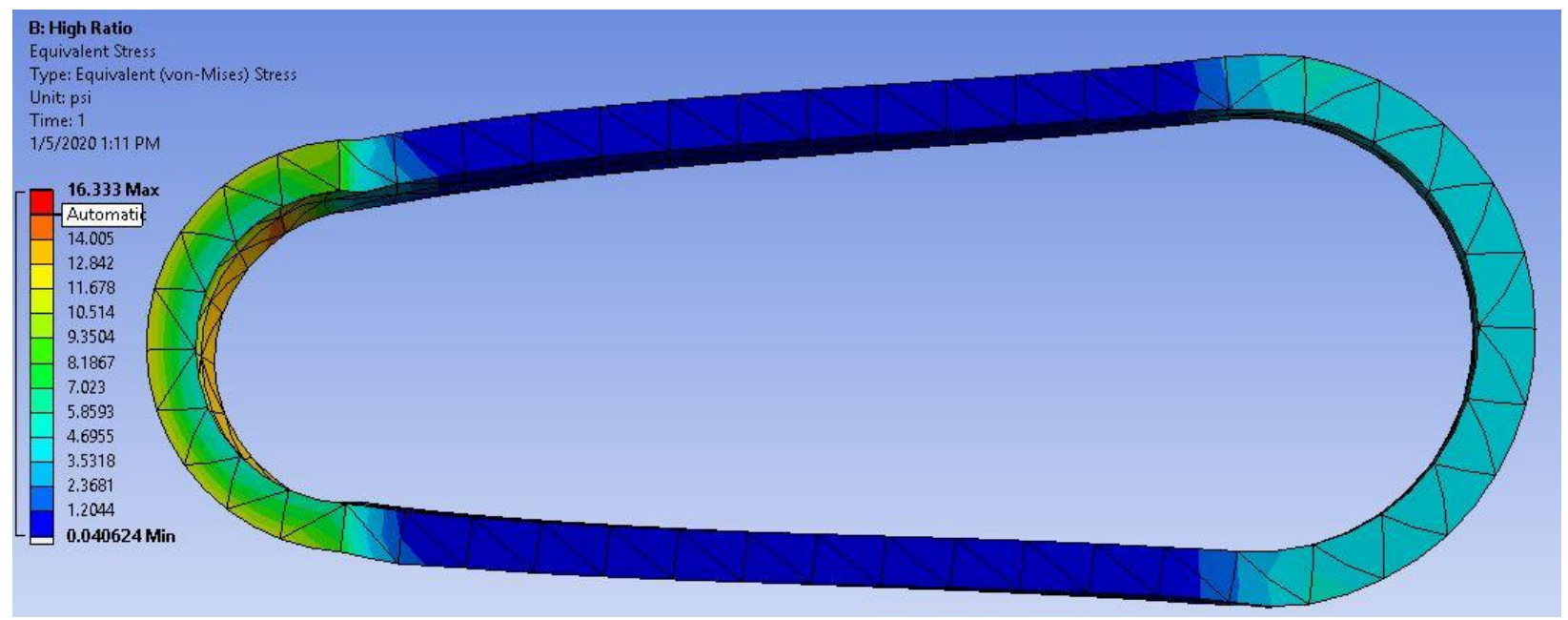

Figure 53: ANSYS High Ratio Stress Distribution. 


\section{Testing Data:}

Testing was initially conducted with the dynamometer to monitor horsepower and the DAQ system to monitor speed at the primary and secondary. After several trails it was concluded that the M19 engine is not powerful enough to efficiently overcome the inertia of the dyno. When loaded by the dyno, the motor couldn't spin up past 3000 RPM. This led to the CVT not being able to shift and being stuck in low ratio. Further tests were conducted with minimal water passing through the dyno, and the CVT being set to shift very easily. Even at this point the motor couldn't overcome the loading of the dyno. To attain proper data on the CVT while shifting, tests were conducted with the dyno removed, and the DAQ system still monitoring speed. Two different CVT setups were tested through shift out and compared to the speed predicted through MATLAB. This comparison is displayed below in Figure 54 and Figure 55. For these tests the vehicle weight was set to 0 in MATLAB to represent a system with no load on it.

The test data is displayed with error bars for the shift ratio and engine speed. These were found based on how the Arduino calculates RPM at the primary and secondary. The code was set up to take a reading every quarter second. Additionally, 6 magnets were placed on each end of the CVT, meaning every 6 magnet counts represents one full revolution. The error bars were found assuming a delta value of $\mp 1$ count per reading. Equation 57 shows that an additional magnet count per time interval would increase the measured angular velocity by 40 RPM. This delta is fixed throughout the operation range, but it should be noted that the percent difference is higher at lower speeds. In the MATLAB code a simple $\mp 40$ RPM was used for the engine speed error bars.

$$
\begin{gathered}
\text { Let: } 1 \text { Count }=\frac{1}{6} \mathrm{rev} \quad \text { And, } \Delta t=0.25 \mathrm{sec} \\
\text { Then, } \frac{\mp 1 \text { Count }}{1 \Delta t}=\frac{\mp \frac{1}{6} \mathrm{rev}}{0.25 \mathrm{sec}}=\mp 40 \mathrm{RPM} \\
\text { Equation 57: Engine RPM Error Bars. }
\end{gathered}
$$

The shift ratio is a bit more complicated because it is calculated based on the primary and secondary measured angular velocities following Equation 58. This means there are two sources of error for this calculation. The fixed $\mp 40$ RPM for either measurement still holds, but the magnitude of its affect depends on how fast the motor is spinning. Recall that the percent error decreases at higher RPM. The maximum error above the measured value occurs when the primary velocity is 40 RPM above and the secondary velocity is 40 RPM below. The maximum error below the measured value occurs when the primary velocity is 40 below and the secondary velocity is 40 RPM above. Thus, the following equations calculate the positive and negative error bars for the shift ratio. The error bar is calculated at each point. It can be observed that as the CVT shifts out, and the secondary RPM increases, the error bars get smaller.

$$
\text { ShiftRatio }=\frac{\text { Primary } R P M}{\text { Secondary } R P M}
$$




$$
X B \text { arPos }=\frac{\text { Primary } R P M+40}{\text { Secondary } R P M-40}-\frac{\operatorname{Primary} R P M}{\text { Secondary } R M}
$$

Equation 59: Shift Ratio Positive Error Bar.

$$
X B \text { arNeg }=-\frac{\text { Primary } R P M-40}{\text { Secondary } R P M+40}+\frac{\text { Primary } R P M}{\text { Secondary } R M}
$$

Equation 60: Shift Ratio Negative Error Bar.

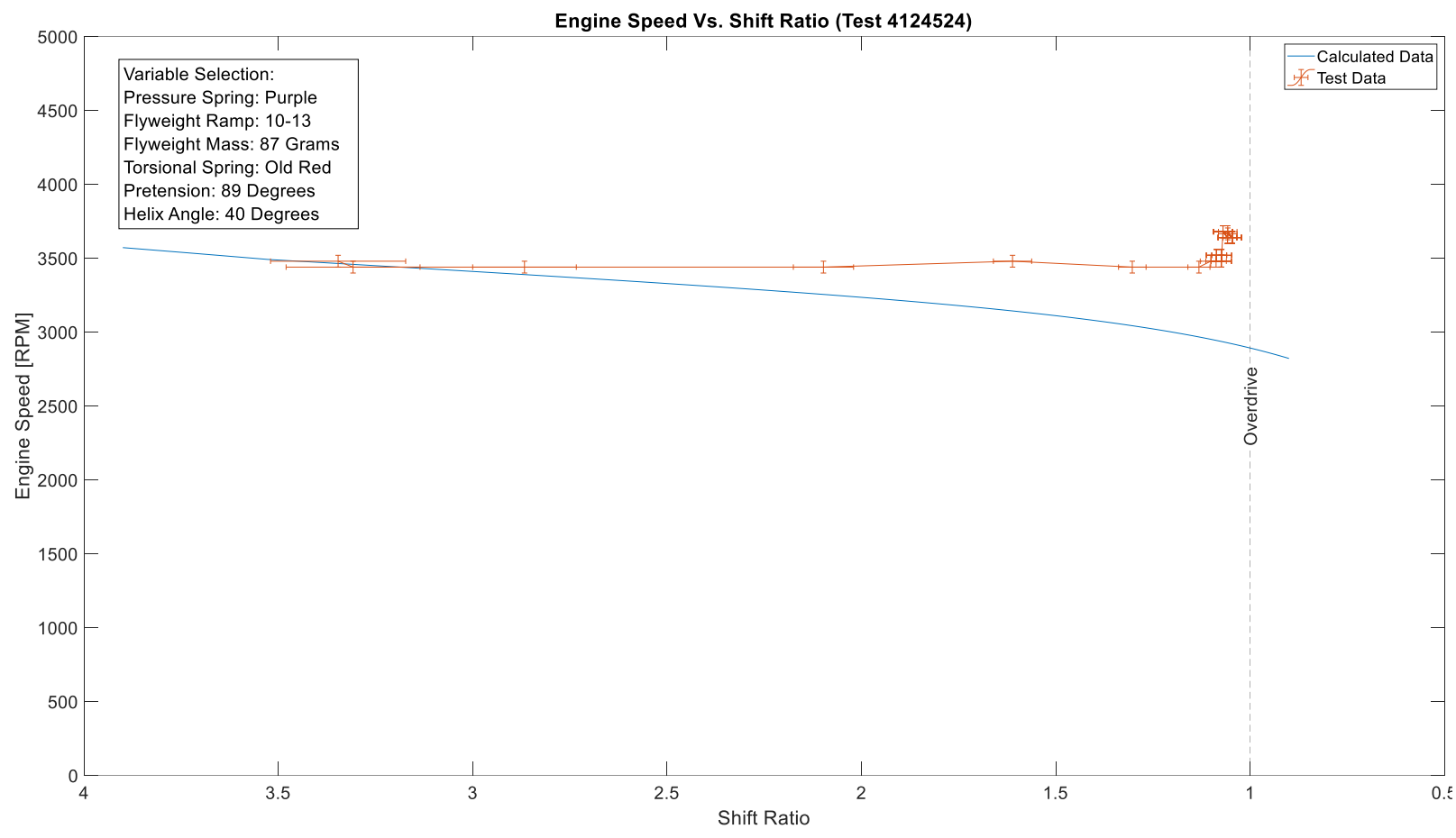

Figure 54: Setup 4124524 Test Comparison. 
Engine Speed Vs. Shift Ratio (Test 4223314)

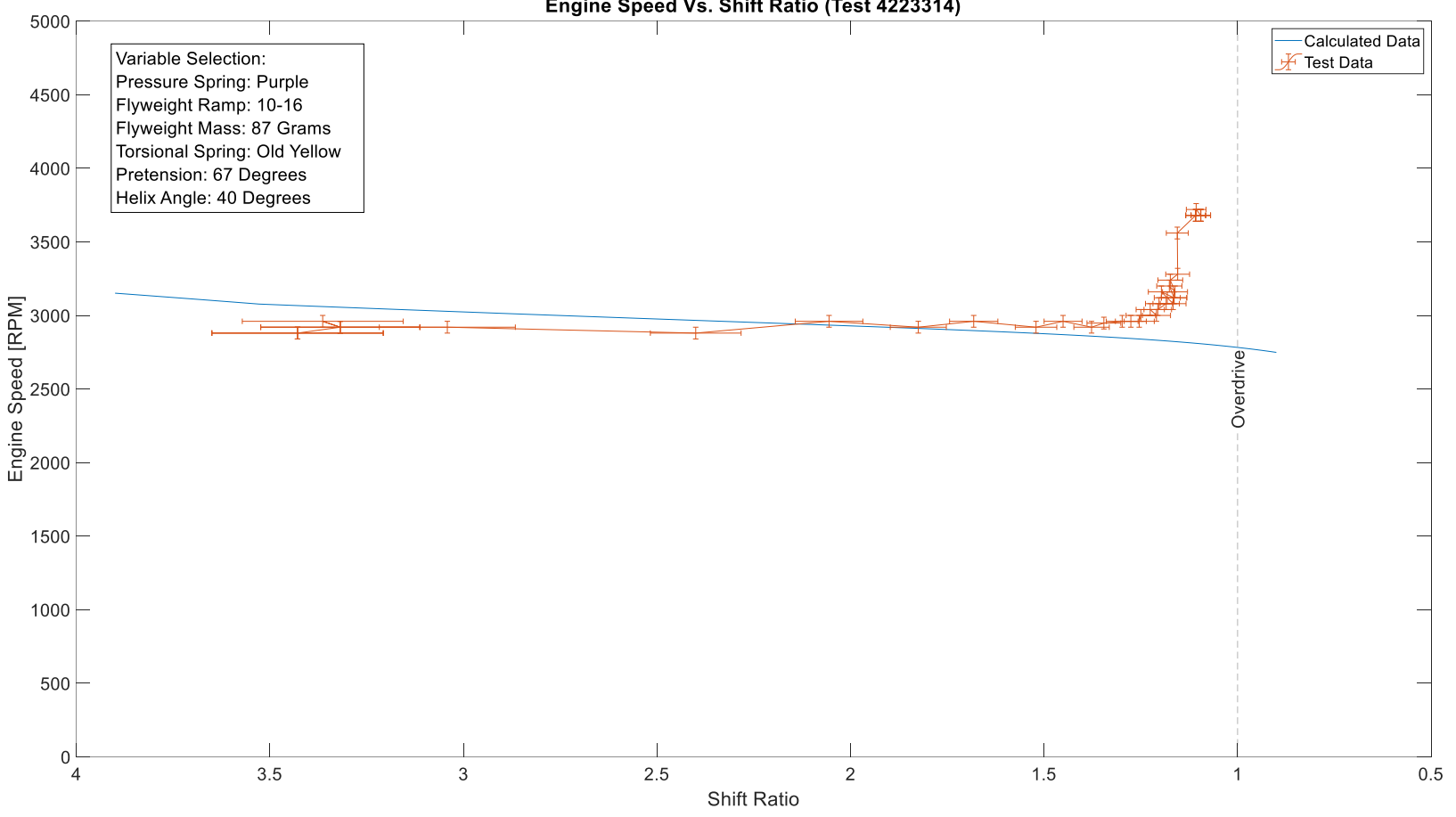

Figure 55: Setup 4223314 Test Comparison. 


\section{Uncertainty Analysis:}

One final level of testing confirmation was conducted. This involves determining the experimental error of values measured in testing. The error of a measurement would usually be considered its deviation from the "true" value. In the case of this research, there isn't a defined true value. Another meaning of experimental error is measuring the deviation between two measured values. More specifically in this application the uncertainty is being measured. This is the total possible error that a measurement might have. Equation 61 details the formula for finding the relative uncertainty. $R$ is the uncertainty, $X$ is the value being measured, $X 1-X n$ are the intermediate measured values, and finally $\Delta \mathrm{X} 1-\Delta X n$ are the resolution of those measurements. This equation was used for determining the uncertainty of linear spring constants and the RPM measured by the DAQ system.

$$
\begin{aligned}
\frac{d R}{R}= & \left\{\left(\Delta X 1 \frac{d X}{X 1}\right)^{2}+\left(\Delta X 2 \frac{d X}{X 2}\right)^{2} \ldots\left(\Delta X n \frac{d X}{X n}\right)^{2}\right\}^{1 / 2} \\
& \text { Equation 61: Uncertainty General Formulation. }
\end{aligned}
$$

In measuring the spring constants there were two measured values, the displacement and the load. The resolution of the linear displacement is 0.0001 inches. The resolution of the force output is $0.1 \mathrm{lbs}$. In the scenario the force load was $81 \mathrm{lbs}$. with a displacement of 1.5 inches. The resultant uncertainty was 0.05013 or $5.01 \%$.

In measuring angular velocity using the Arduino setup, there were two measured values, the Arduino board time, and the number of magnet passes. The resolution of the board time is 16 $\mathrm{MHz}$ or $6.25 \mathrm{E}-15$. The resolution of the number of magnet passes is 1 divided by the total number of magnet passes in that interval of board time. Reducing this expression yields the following, with $\omega$ representing angular velocity and BT representing board time. Board time is fixed at 0.25 seconds.

$$
\Delta N=\frac{2.5}{\omega * B T}
$$

Given the expression for $\Delta \mathrm{N}$ it shows that uncertainty will decrease as angular velocity increases. This makes sense because as more magnet passes go by per second it is less impactful if a magnet pass is missed. At the shift out point, 3600 RPM, the uncertainty is 0.002777 or $.277 \%$. 


\section{Conclusions:}

The purpose of modeling the CVT belt in ANSYS was to determine if the belt tension calculated in MATLAB was reasonable. Initial comparisons were promising, yielding between 1235 percent error at either end of the belt. This confirms that the tensions calculated were of the right magnitude. There are several factors that contributed to this error. Primarily, the biggest factor was the necessity to estimate the material properties of the belt by comparing to a similar CVT belt. The CVTech belt is wider and could possibly be made of a different material. Additionally, the CVTech belt doesn't have steel chording for added strength like the Enduro 100 belt does. Finally, the density of the belt was determined without considering the cogs in the belt. Observing these would have increased the density of the material and contributed to the material properties changing. With these considerations, the tensions calculated in MATLAB were determined to be acceptable.

Testing results showed a strong correlation between predicted and observed performance. Initial shift out speed predicted by MATLAB matched test results within 200 RPM. As the CVT approached overdrive the difference increased slightly. This is most likely because the system wasn't under load. In testing it was observed that once the sheaves started to move, they would shift all the way to over drive in under 1 second. This implies it is very likely the motor was spinning faster than required to continue shifting. If the system was under load this shift time would be drawn out longer, and the motor RPM would decrease as it approached overdrive. This would cause the shift curves between testing and MATLAB to be nearly identical. For future testing a smaller dynamometer designed for an approximately $10 \mathrm{hp}$ motor is recommended. With these considerations the MATLAB program is suitable for predicting actual vehicle performance.

Using the MATLAB program to cycle through all possible setups significantly cuts down on the time required to find a setup with optimal performance characteristics. The recommended use of this program is to first make all measurements for constants required for calculations. Next, consider the weight of the vehicle, and the terrain that it will be encountering. If there will be a variety of hills, mud, or jumps, it would be wise to increase the required transferrable torque to ensure proper power transfer and hill climbing ability. This will also account for shock loading after being air-born. Next the performance requirements for a setup should be determined. These will be dependent on the motor that is being used, and the speed at which it produces maximum torque and power. From this information, the desired shift and engagement speeds can be set.

Future testing will require use of a mobile DAQ system capable of monitoring primary and secondary speed on a moving vehicle. With this, a setup with predicted performance can be found and then compared to results on the moving vehicle. This will also allow more precise comparisons on how the vehicle weight and hill angle affect performance. This could have been more closely checked if testing with the dynamometer was possible, because a specific load could have been

placed on the motor. Overall, this system will allow the Baja SAE ${ }^{\circledR}$ team to set their CVT closer to their specific performance objectives before any vehicle testing is needed. 


\section{Contributions:}

The research conducted for this thesis required work in several areas. These areas cover new research efforts, physical testing systems, and new methodologies for the WVU Baja team.

\section{CVT Tuning Research:}

The team will now have precise objectives in mind when tuning their CVTs. Having specific goals and understanding what factors are most important will guide the tuning process. These objectives were defined based on research of CVTs in racing applications, specifically snowmobiling.

\section{Spring Testing Rig:}

The spring testing rig was manufactured to be able to accurately determine spring constants for the CVT. This was required for this research because the GX9 CVT manufacturer did not provide any constants for the springs. The spring rig will also be useful for determining how the age of springs affects its spring constants. Interestingly, the older springs tested had a higher spring constant than the newer springs of the same color.

\section{CAD Model:}

The CVT CAD model will be useful for a variety of reasons. The assembly was mated in a way that the entire system moves realistically, including the belt. The user could move the sheave at the primary and observe how the belt radii will change, and how the secondary will twist and shift. This is a great learning tool for someone who needs help understanding the moving parts in the GX9 CVT. Furthermore, the model is a resource for fitment checks when designing the car in CAD. This included intricate measurements such as designing the CVT cover. Finally, the model is used to perform FEA analysis. This will be useful to further research on more complicated belt dynamics.

\section{MATLAB Setup Analysis:}

The primary contribution of this research is the MATLAB code that was created. This code will be used to determine optimal setups without requiring physical testing. This will cut down on the time required to determine the best CVT setup for any given powertrain. Furthermore, this analysis considered many things that previous research did not. These include centrifugal forces of the belt and flyweight links, and torque loading from the weight of the vehicle. This research also only produces results based on the given setup combinations. This means that it tells the user the best setup based on what is available for use.

\section{Testing Setup:}

The last step of this research was confirmatory testing to determine accuracy of calculations from MATLAB. A complete dynamometer testing setup was put together in conjunction with the WVU Formula team. This included the design of a custom table for mounting the motor, CVT, reduction pulley system, and the water brake. The table was designed in solid works with help from the 2018-19 Baja team. The system has the capability of monitoring power and torque production from the motor at the brake. It was found that the brake itself had too much inertia for 
the M19 to motor to push effectively. However, given the flexible nature of the reduction system, a smaller dyno would work well on this table. When a new dyno more fitting for this motor is purchased there should be no issues using it with this table.

Finally, an Arduino based data acquisition system was put together by the 2019-20 Baja team for the purpose of monitoring RPM data of the primary and secondary. This system is mounted onto the table as well, monitoring both power output and speed data throughout operation of the system. This speed data is the primary means of comparing MATLAB calculations to real world testing data. In the future when reliable power output data can be gathered these values can be compared as well. 


\section{Bibliography:}

Aaen, Olav. Olav Aaen's Clutch Tuning Handbook. 2015.

Aulakh, Jot Singh. "Development of simulation approach for CVT tuning using dual level genetic algorithm." Cogent Engineering (2017): Vol. 4. Web.

Aztec Baja. AztecBaja: Drive-Train. 2017. Website. 10 July 2019.

Briggs \& Stratton Racing. Briggsracing.com. 2019. 2019.

Gaged Engineering. Gagedengineering.com. 2019. 2019.

Hibbeler, R. C. Engineering Mechanics Statics Thirteenth Edition. Upper Sadddle River: Pearson Prentice Hall, 2013.

Holloway, Hilton and Martin Buckley. 20th Century Cars. Carlton, 2002.

Johnson, Jack L. Designers' Handbook for Electrohydraulic Servo and Proportional Systems. IDAS Engineering Inc., 2000.

Land \& Sea, Inc. https://www.dynomitedynamometer.com/. 281 2019. 2019.

Messick, Matthew James. "An Experimentally-Validated V-Belt Model for Axial Force and Efficiency in a Continuously Variable Transmission." Thesis. 2018. Document. 082019.

Moradmand, Jamshid, Russell Marcks and Tom Looker. Statics for Engineering Technology. Ohio Digital Bookshelf, n.d.

Pennslyvania Tech. http://forums.bajasae.net. 10 2016. Forum. 82019.

PSU. Adaptivemap.ma.psu.edu. n.d. 2019.

Richard G. Budynas, J. Keith Nisbett. Shigley's Mechnical Engineering Design, Tenth Edition. McGraw Hill Education, 2015.

Strang, Gilbert. Introduction to Linear Algebra 5th Edition. Cambridge Press, n.d. PDF. 


\section{Appendix:}

\section{Spring Test Rig Data:}

\begin{tabular}{|c|c|c|c|c|c|c|c|c|c|c|c|c|c|}
\hline & \multicolumn{13}{|c|}{ Old Black Pressure Spring Compression } \\
\hline Displacement (in.) & Trial 1 & Trial 2 & Trial 3 & Trial 4 & Trial 5 & Trial 6 & Trial 7 & Trial 8 & Trial 9 & Trial 10 & Average Force (Ibs) & Spring Constant (Ibs/in) & Least Squares Constant (Ibs/in) \\
\hline 0.25 & 9.4 & 9.6 & 9.8 & 10 & 9.8 & 19 & 9.6 & 9.6 & 9.8 & 10 & 10.66 & 42.64 & \multirow{7}{*}{43.75} \\
\hline 0.5 & 20.4 & 20.6 & 20.4 & 20.8 & 20.2 & 20.6 & 20.2 & 20.2 & 20.6 & 20.6 & 20.46 & 40.92 & \\
\hline 0.75 & 31 & 31 & 31.4 & 31.8 & 31.2 & 31.4 & 31.2 & 31 & 31.4 & 31.4 & 31.28 & 41.70666667 & \\
\hline 1 & 42.2 & 42.2 & 42.4 & 42.6 & 42.4 & 42.4 & 42.2 & 42 & 42.4 & 42.4 & 42.32 & 42.32 & \\
\hline 1.25 & 53.8 & 53.8 & 53.8 & 54.2 & 53.8 & 53.8 & 53.4 & 53.4 & 54 & 54.2 & 53.82 & 43.056 & \\
\hline 1.5 & 65.6 & 65.6 & 66 & 66.6 & 66 & 66 & 66 & 65.6 & 66.2 & 66 & 65.96 & 43.97333333 & \\
\hline \multirow[t]{4}{*}{1.75} & 78.6 & 78.6 & 78.6 & 79.4 & 78.6 & 78.6 & 78.8 & 78.6 & 79 & 79.2 & 78.8 & 45.02857143 & \\
\hline & & & & & & & & & & & & & . \\
\hline & & & & & & & & & & & & & \\
\hline & \multicolumn{13}{|c|}{ New Black Pressure Spring Compression } \\
\hline Displacement (in.) & Trial 1 & Trial 2 & Trial 3 & Trial 4 & Trial 5 & Trial 6 & Trial 7 & Trial 8 & 3 Trial 9 & Trial 10 & Average Force (lbs) & Spring Constant (lbs/in) & Least Squares Constant (Ibs/in) \\
\hline 0.25 & 8.2 & 8 & 8.8 & 8.8 & 8.6 & 8.6 & 8.8 & 8.8 & 8.4 & 9.2 & 8.62 & 34.48 & \multirow{7}{*}{42.71} \\
\hline 0.5 & 18.8 & 18.8 & 19.6 & 19.6 & 19.4 & 19.4 & 19.6 & 19.6 & 19.2 & 19.8 & 19.38 & 38.76 & \\
\hline 0.75 & 29.6 & 29.4 & 30.4 & 30.4 & 30.2 & 30.2 & 30.4 & 30.4 & 30 & 30.8 & 30.18 & 40.24 & \\
\hline 1 & 40.6 & 40.6 & 41.4 & 41.2 & 41.2 & 41.2 & 41.4 & 41.4 & 41 & 41.6 & 41.16 & 41.16 & \\
\hline 1.25 & 52 & 51.8 & 52.8 & 52.8 & 52.6 & 52.6 & 52.8 & 52.8 & 52.4 & 53 & 52.56 & 42.048 & \\
\hline 1.5 & 64.2 & 64 & 65 & 64.8 & 64.8 & 64.8 & 64.8 & 64.8 & 64.4 & 65.2 & 64.68 & 43.12 & \\
\hline 1.75 & 76.8 & 76.6 & 77.6 & 77.6 & 77.4 & 77.4 & 77.6 & 77.6 & 77 & 78 & 77.36 & 44.20571429 & \\
\hline
\end{tabular}

\begin{tabular}{|c|c|c|c|c|c|c|c|c|c|c|c|c|c|}
\hline & \multicolumn{13}{|c|}{ New Purple Pressure Spring Compression } \\
\hline Displacement (in.) & Trial 1 & Trial 2 & Trial 3 & Trial 4 & Trial 5 & Trial 6 & Trial 7 & Trial 8 & Trial 9 & Trial 10 & Average Force (Ibs) & \begin{tabular}{|l|} 
Spring Constant (Ibs/in) \\
\end{tabular} & Least Squares Constant (Ibs/in) \\
\hline 0.25 & 18 & 19 & 19.4 & 19.4 & 17.6 & 16.4 & 18.4 & 17 & 19 & 17.4 & 18.16 & 72.64 & \multirow{7}{*}{$x_{1} x_{1}$} \\
\hline 0.5 & 39.6 & 40.8 & 41.2 & 41.4 & 39.4 & 38.2 & 40.2 & 39.2 & 41.2 & 39.2 & 40.04 & 80.08 & \\
\hline 0.75 & 61.6 & 62.6 & 63 & 63 & 61.2 & 60.2 & 62 & 61.4 & 61.6 & 61.4 & 61.8 & 82.4 & \\
\hline 1 & 83.4 & 84.4 & 85 & 85 & 83 & 82 & 84 & 83.2 & 80.4 & 83.4 & 83.38 & 83.38 & \\
\hline 1.25 & 105.6 & 106.6 & 101.2 & 107.4 & 105 & 104 & 106 & 104.8 & 108 & 105.8 & 105.44 & 84.352 & \\
\hline 1.5 & 131 & 132 & 133 & 132.8 & 130.2 & 129.4 & 131.2 & 129.8 & 127.2 & 131.2 & 130.78 & 87.186666667 & \\
\hline 1.75 & & & & & & & & & & & & & \\
\hline
\end{tabular}

\begin{tabular}{|c|c|c|c|c|c|c|c|c|c|c|c|c|c|c|c|}
\hline & \multicolumn{10}{|c|}{ New Orange Pressure Spring Compression } \\
\hline Displacement (in.) & Trial 1 & Trial 2 & Trial 3 & Trial 4 & \multicolumn{1}{|c|}{ Trial 5 } & Trial 6 & Trial 7 & Trial 8 & Trial 9 & Trial 10 & Average Force (lbs) & Spring Constant (Ibs/in) & Least Squares Constant (Ibs/in) \\
\hline & 16.4 & 17.2 & 16.6 & 17.4 & 19 & 19 & 18 & 17.6 & 16.8 & 17 & 17.5 & 70 \\
\hline 0.25 & 35.6 & 36.4 & 35.8 & 36.6 & 38.2 & 38 & 37.4 & 36.6 & 36 & 35.6 & 36.62 & 73.24 \\
\hline 0.5 & 55 & 55.8 & 55.4 & 56.2 & 57.4 & 57.8 & 57 & 56.4 & 55.6 & 55.88 & 56.248 & 74.99733333 \\
\hline 0.75 & 76.4 & 77.4 & 76.6 & 77 & 79.4 & 78.6 & 78.4 & 78 & 77 & 76.6 & 77.54 & 77.54 \\
\hline 1 & 99 & 100 & 99 & 100.2 & 102.6 & 102 & 101.6 & 100.2 & 99.4 & 99.4 & 100.34 & 80.272 \\
\hline 1.25 & 122.8 & 123.6 & 122.4 & 124.2 & 125.8 & 125.8 & 125.6 & 124 & 123.4 & 126 & 124.36 & 82.90666667 \\
\hline 1.5 & & & & & & & & & & & & \\
\hline 1.75 & & & & & & & & & & & & & & & \\
\hline
\end{tabular}




\begin{tabular}{|c|c|c|c|c|c|c|c|c|c|c|c|c|c|}
\hline & \multicolumn{13}{|c|}{ New Red Spring Compression } \\
\hline \begin{tabular}{|l|l} 
Displacement (in.) \\
\end{tabular} & Trial 1 & \begin{tabular}{|c|} 
Trial 2 \\
\end{tabular} & Trial 3 & $\begin{array}{c}\text { Trial } 4 \\
\end{array}$ & Trial 5 & $\begin{array}{l}\text { Trial } 6 \\
\end{array}$ & $\begin{array}{ll}\text { Trial } 7 \\
\end{array}$ & Trial 8 & \begin{tabular}{|l} 
Trial 9 \\
\end{tabular} & Trial 10 & Average Force (lbs) & Spring Constant $(\mathrm{lbs} / \mathrm{in})$ & \multirow[t]{9}{*}{ 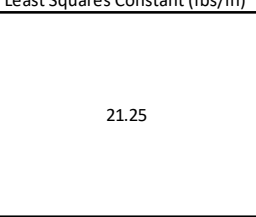 } \\
\hline 0.25 & 4.6 & 4.8 & 4.6 & 5 & 4.6 & 4.4 & 4.6 & 5 & 4.6 & 4.4 & 4.66 & 18.64 & \\
\hline 0.5 & 10 & 10.2 & 9.8 & 10.2 & 10 & 9.8 & 10 & 10.4 & 10 & 10 & 10.04 & 20.08 & \\
\hline 0.75 & 15.4 & 15.8 & 15.6 & 15.2 & 15.4 & 15.2 & 15.4 & 15.8 & 15.4 & 15.4 & 15.46 & 20.61333333 & \\
\hline 1 & 21 & 21.4 & 21.2 & 21.2 & 20.8 & 20.6 & 21 & 21.4 & 21 & 20.6 & 21.02 & 21.02 & \\
\hline 1.25 & 26.8 & 27 & 26 & 26.4 & 26.2 & 26.2 & 26.4 & 27 & 26.8 & 26 & 26.48 & 21.184 & \\
\hline 1.5 & 32.4 & 32.4 & 31.6 & 32.2 & 32 & 31.8 & 31.8 & 32.4 & 32.6 & 31.6 & 32.08 & 21.38666667 & \\
\hline 1.75 & 37.4 & 38 & 36.8 & 37.2 & 37.8 & 37.6 & 37.4 & 37.6 & 37.4 & 37.2 & 37.44 & 21.39428571 & \\
\hline 2 & 42.2 & 43.4 & 42.6 & 43.2 & 42.8 & 43 & 42.8 & 43.2 & 42.4 & 41.6 & 42.72 & 21.36 & \\
\hline
\end{tabular}

\begin{tabular}{|c|c|c|c|c|c|c|c|c|c|c|c|c|c|}
\hline & \multicolumn{10}{|c|}{ Old Red Spring Compression } \\
\hline Displacement (in.)) & Trial 1 & Trial 2 & Trial 3 & Trial 4 & Trial 5 & Trial 6 & Trial 7 & Trial 8 & Trial 9 & Trial 10 & Average Force (lbs) & Spring Constant (lbs/in) & Least Squares Constant (lbs/in) \\
\hline 0.25 & 3.8 & 4.2 & 4.2 & 3.8 & 3.8 & 4 & 4.2 & 5 & 4.4 & 4.2 & 4.16 & 16.64 & \\
\hline 0.5 & 10.6 & 10.8 & 10.2 & 10.4 & 10.6 & 10.6 & 11 & 11.6 & 11.4 & 11 & 10.82 & 21.64 \\
\hline 0.75 & 16.6 & 17 & 16.8 & 17.2 & 17.2 & 17 & 17.6 & 18.2 & 17.6 & 18.6 & 17.38 & 23.17333333 \\
\hline 1 & 22.4 & 23 & 23.2 & 23.8 & 23.6 & 23.2 & 24.2 & 25 & 25.2 & 25 & 23.86 & 23.86 \\
\hline 1.25 & 31.2 & 31.4 & 29.8 & 30 & 30.4 & 31.2 & 31.6 & 31.6 & 31.2 & 31.8 & 31.02 & 24.816 & \\
\hline 1.5 & 35.6 & 37.6 & 37.2 & 37.4 & 37.4 & 37 & 36.8 & 38.2 & 38.4 & 38.2 & 37.38 & 24.92 & 25.23 \\
\hline 1.75 & 44.8 & 45 & 42.2 & 45 & 44 & 42.4 & 45.6 & 45.6 & 45.8 & 45.6 & 44.6 & 25.48571429 \\
\hline 2 & 54.4 & 52.4 & 51 & 50.6 & 49.6 & 53.6 & 53.4 & 54.4 & 54 & 54 & 52.74 & 26.37 & \\
\hline
\end{tabular}

\begin{tabular}{|c|c|c|c|c|c|c|c|c|c|c|c|c|c|}
\hline & \multicolumn{13}{|c|}{ Old Yellow Spring Compression } \\
\hline \begin{tabular}{|l} 
Displacement (in.) \\
\end{tabular} & Trial 1 & Trial 2 & Trial 3 & Trial 4 & Trial 5 & Trial 6 & Trial 7 & Trial 8 & Trial 9 & Trial 10 & Average Force (lbs & Spring Constant (lbs/in) & \begin{tabular}{|l|l} 
Least Squares Constant (lbs/in) \\
\end{tabular} \\
\hline 0.25 & 4.2 & 4.6 & 4.2 & 4.4 & 4.4 & 4.6 & 4.8 & 5 & 4.8 & 4.8 & 4.58 & 18.32 & \multirow{8}{*}{22.75} \\
\hline 0.5 & 9.6 & 10 & 9.8 & 9.8 & 9.8 & 10 & 10.2 & 10.4 & 10.2 & 10.2 & 10 & 20 & \\
\hline 0.75 & 15 & 15.2 & 15 & 15.2 & 15.2 & 15.4 & 15.6 & 16 & 15.8 & 15.8 & 15.42 & 20.56 & \\
\hline 1 & 20.4 & 20.6 & 20.4 & 20.6 & 20.6 & 21 & 21 & 21.2 & 21.2 & 21.2 & 20.82 & 20.82 & \\
\hline 1.25 & 26.2 & 26.6 & 26.4 & 26.6 & 26.4 & 26.6 & 27 & 27.4 & 26.8 & 27.2 & 26.72 & 21.376 & \\
\hline 1.5 & 33 & 33 & 33 & 32.8 & 33.4 & 33.4 & 33.8 & 34.2 & 33.8 & 33.4 & 33.38 & 22.25333333 & \\
\hline 1.75 & 41.6 & 39.8 & 40.2 & 41 & 40.6 & 40.2 & 41.6 & 41 & 41.6 & 39.4 & 40.7 & 23.25714286 & \\
\hline 2 & 47 & 47.8 & 48.8 & 48.2 & 48.2 & 48.8 & 48 & 48.6 & 49.6 & 49 & 48.4 & 24.2 & \\
\hline
\end{tabular}

\begin{tabular}{|c|c|c|c|c|c|c|c|c|c|c|c|c|c|}
\hline & \multicolumn{13}{|c|}{ New Yellow Spring Compression } \\
\hline Displacement (in.) & Trial 1 & Trial 2 & Trial 3 & Trial 4 & Trial 5 & Trial 6 & Trial 7 & Trial 8 & Trial 9 & Trial 10 & Average Force (lbs) & Spring Constant (lbs/in) & Least Squares Constant (lbs/in) \\
\hline 0.25 & 3 & 3.4 & 3.4 & 3.2 & 3.4 & 3.4 & 3.2 & 3.4 & 3.4 & 3.4 & 3.32 & 13.28 & \multirow{8}{*}{14.65} \\
\hline 0.5 & 7 & 7.4 & 7.2 & 7.2 & 7.2 & 7.2 & 7.2 & 7.2 & 7.2 & 7.2 & 7.2 & 14.4 & \\
\hline 0.75 & 10.8 & 11.2 & 11.2 & 11 & 11 & 11 & 11 & 11.2 & 11 & 11 & 11.04 & 14.72 & \\
\hline 1 & 14.8 & 15 & 15 & 14.8 & 15 & 15 & 15 & 15 & 15 & 15 & 14.96 & 14.96 & \\
\hline 1.25 & 18.6 & 19 & 19 & 18.6 & 18.8 & 18.6 & 18.4 & 18.6 & 18.4 & 18.2 & 18.62 & 14.896 & \\
\hline 1.5 & 22.4 & 22.8 & 22.6 & 22.2 & 22.6 & 22.2 & 22.2 & 22.4 & 21 & 21.2 & 22.16 & 14.77333333 & \\
\hline 1.75 & 25.8 & 26 & 26 & 25.8 & 25.8 & 25.4 & 24.6 & 24.4 & 24.4 & 24.4 & 25.26 & 14.43428571 & \\
\hline 2 & 29.8 & 30.4 & 29.8 & 29.6 & 29.6 & 29.6 & 28 & 28.8 & 28.4 & 28 & 29.2 & $\begin{array}{ll}14.6 \\
\end{array}$ & \\
\hline
\end{tabular}




\section{MATLAB Script: \\ Main Script:}

\%WVU BAJA CVT Analysis

\%Sean Skinnr

Fall 18 - Spring 20

Clc

clear

close all

응 Defind Variables

\%SCRIPT

$\mathrm{n}=100 ; \quad$ Nomber of points during each

phase

Vehicle Mass

$\mathrm{WV}=350 ; \quad \therefore \mathrm{l}$ ibs

\%Hill Angle

Gamma $=20 ; \quad$ \%Degrees

Engine

Pee $=7.5 ; \quad$ Engine power engagment

(hp)

Pes $=8.5 ; \quad$ oEngine power shift (hp)

Wde $=2800 ; \quad$ oDesired engagement angular velocity of engine (RPM)

Wds = 3600; $\quad$ oDesired shift Angular

Velocity of engine (RPM)

oTires

RTire = 11; $\quad$ otire Radius (in.)

\%Gear Box

$\mathrm{Rgb}=1 / 7$;

oGear Box Reduction Ratio

BELT

$\mathrm{CC}=10 ;$

\%Center to center (in.)

$\mathrm{U}=0.13$;

o.Shigleys Pg 915. Values

from Gates Rubber Company

MBelt $=0.01579 / 32.174 ; \quad$ Mass per unit

length (Sluggs/in)

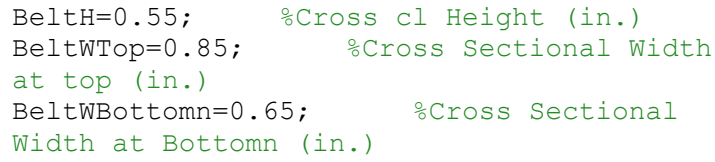

\%PRIMARY

\% Sheaves

Theta $=23 ; \quad \therefore B e l t$ groove primary

(Degrees)

$\mathrm{X} 1 \max =0.8 ; \quad \quad$ oTotal sheave displacement

(in.)

Rpmin $=0.75+($ BeltH $/ 2) ; \quad$ \%Minimum pitch

radius (in.)

Rpmax $=3-(\operatorname{BeltH} / 2) ; \quad \frac{\circ}{0}$ Maximum pitch

radius (in.)

oPressure Spring
Kp=xlsread ('CVT Constants 2',1, 'N2:Q2'); oLinar spring rates (lbf/in.) [Set: A] $\mathrm{Xp0}=0.975 ; \quad$ \%Intalled pretenion (in.)

Flyweights

Ufly=0.002; $\quad$ Coefficient of friction between flyweight ramps and flyweight roller bearings

Deltastart $=x l$ sread ( 'CVT Constants.

2',1,'D2:F2')'; $\quad$ oRamp angles (Degrees)

[Set: B]

Deltaend=xlsread ('CVT Constants

2', 1, 'D3:F3')' ;

FlatAngle $=x l s r e a d($ 'CVT Constants

2', 1, 'D9:F9')';

IO=.05; $\quad$ Flat angle constant. Affects initial shift out point

Mfly=xlsread ('CVT Constants

2', 1, 'D4:I4').*(4/14593.903);

:Flyweight Mass for four arm (Slugs) [Set: C ]

Rfly0 = 1.875; $\quad$ Initial radius (in.)

(1.34)

RflyMax=2.345; Full Shift Radius (in.)

$(1.815)$

Mlink=21 ** (4/14593.903);

Link mass

for four arms (Sluggs)

ECONDARY

○Sheaves

Phi $=23 ; \quad$ \%Belt groove secondary

(Degrees)

$\mathrm{x} 2 \max =0.8 ; \quad$ oTotal sheave displacement

(in.)

Rsmin $=\operatorname{Rpmax}^{*} 0.9 ; \quad$ oMinimum pitch radius

(in.)

Rsmax $=$ Rpmin*3.9; $\quad$ \%Maximum pitch

radius (in.)

oTorsional Spring

xto $=1.44 ; \quad$ Ontalled linar Length

(in.)

Kt=xlsread ('CVT Constants 2',1, 'N3:Q3');

oLinar spring rates (lbf/in.) [Set: D]

Yt0 $=x$ lsread ('CVT Constants 2',1,'D7:I7');

ontalled twists (Degrees) [Set: E]

Ymax=40; $\quad$ oTotal Secondary Twist

(Degrees)

Lambda=xlsread ('CVT Constants 2',1, 'N4:04') ;

oTorsional spring rates (in*lbf/Degree.)

[Set: $\mathrm{F}$ ]

$\% \mathrm{Helix}$

Etastart $=\mathrm{xls}$ lead $($ 'CVT Constants

2',1,'D5:H5')'; \%Ramp angles (Degrees)

[Set: G]

Etaend=xlsread ('CVT Constants

2', 1, 'D6:H6') ';

$\mathrm{Rr}=1.455 ; \quad \frac{\circ}{2} \quad$ Ramp radius (in.)

\%믄 Culated Variables:

Engine

\%Design Hp 
Hde $=\mathrm{Pee}^{\star} \mathrm{KS}{ }^{\star} \mathrm{Nd} ; \quad$ Design power engagement (hp) (Eq 17-19 Shigley's)

$\mathrm{Hds}=\mathrm{Pes}{ }^{\star} \mathrm{Ks}{ }^{\star} \mathrm{Nd} ; \quad$ \%Design power during shift (hp)

oVehicle Weight Torque Load

$\mathrm{TV}=\left(\mathrm{WV}{ }^{*} \mathrm{~S}\right.$ ind (Gamma) ${ }^{*} \mathrm{RT}$ ire $\left.{ }^{*} \mathrm{Rgb}\right) / 12$;

\%ft. *lbs

\%Belt

Belt $\mathrm{SH}=(($ ( (BeltwTop-

BeltWBottomn $\left.\left.) / 2)^{\wedge} 2\right)+\left(\operatorname{BeltH}{ }^{\wedge} 2\right)\right)^{\wedge}(.5)$;

oside Face Height (in.)

\%Flyweights

Rfly=linspace (Rflyo, RflyMax, n) ;

\%Flyweight ramp radius through shift (in.)

[Vector]

$\operatorname{Rlink}=1.625+(0.625 . * \operatorname{acos}((\operatorname{Rr}-1.625) . / 1.25))$;

\%Flyweight Ramp Angles

Delta=FlatAngle. *ones (length (Deltastart), n) ;

for $i=1$ : size (Delta, 1)

Delta $(i,((I 0 * n)+1): n)=l$ inspace (Deltastart $(i)$

, Deltaend (i), $(\mathrm{n}-(\mathrm{I} 0 * \mathrm{n})))$; $i=i+1$;

end

osheaves

$\mathrm{Rp}=$ linspace (Rpmin, Rpmax, n) ; $\quad$ orimary

radius through shift (in.) [Vector]

Rs = linspace (Rsmax, Rsmin, n);

osecondary radius through shift (in.)

[Vector]

\%Shift Ratio

$\mathrm{R}=\mathrm{Rs}$. / Rp ;

oshift Distances

$\mathrm{X} 1=1$ inspace $(0, \mathrm{X} 1 \mathrm{max}, \mathrm{n}) ; \quad$ oprimary

sheave displacement through shift (in.)

[Vector]

$\mathrm{X} 2=1$ inspace $(0, \mathrm{x} 2 \max , \mathrm{n})$;

o Secondary sheave displacement through shfit (in.) [Vector]

$\mathrm{Y} 2=1$ inspace $(0, \operatorname{Ymax}, \mathrm{n})$;

\% Secondary sheave twist through shift

(Degrees) [Vector]

ㄱelix

Eta $=$ Etastart . ${ }^{*}$ ones (length (Etastart), n) ;

for $i=1: \operatorname{size}($ Eta, 1$)$

$\operatorname{Eta}(i,:)=\operatorname{linspace}(\operatorname{Eta}(i, 1), \operatorname{Etaend}(i, 1), n)$; $i=i+1$;

end

Effective Coefficient of Friction

$\mathrm{Uep}=\mathrm{U} /(\operatorname{sind}(($ Theta $) / 2)) ; \quad \frac{\text { Primary }}{}$

effective coefficient of friction

Ues $=\mathrm{U} /($ sind $((\mathrm{Phi}) / 2)) ; \quad$ Secondary

effective coefficient of friciton

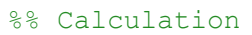

ㅇhift

[TpShift, TsShift, AlphaShift, BetaShift, TplShi ft, Tp0Shift, CpxShift, FpxShift, FpsShift, Fflyx Shift, FflyShift, wpcShift,MPMaxShift, FxtsShif t, FytsShift, FHelixShift, FsShift, FsxShift, Csx Shift, Ts0Shift, Ts1Shift,MSMaxShift, EffsShift
, Setupshift] =

ShiftV8 ( $\mathrm{n}$, Hds, Wds , Tv, Rp, Rs, R, X1 , X2 , Y2 , U, Uep, Ues, MBelt, BeltSH, Ufly, cc, Kc, Theta, Phi, Kp, Xp0 , Delta, Mfly, Rfly, Mlink, Rlink, Xt0, Kt, Yto, Lamb da, Eta, Rr);

\%Engagement Point

[TpEngage, TsEngage, Alpha, Beta, Tp1Engage, Tp0E ngage, CpxEngage, FpxEngage, FpsEngage, FflyxEng age, FflyEngage, wpcEngage, MPMaxEngage, FxtsEng age, FytsEngage, FHelixEngage, FsxEngage, CsxEng age, Ts0Engage, TslEngage, MSMaxEngage, EffsEnga ge, SetupEngage ] =

EngagementV2 ( $\mathrm{n}$, SetupShift, Hde, Wde, Tv, Rp, Rs, R , U, Uep, Ues, MBelt, Ufly, cc, Theta, Phi, Kp, Xp0, De lta, Mfly, Rfly, Mlink, Rlink, xto , Kt, Yto, Lambda, Eta, Rr);

-Truncating Unacceptable Setups

\%Primary

Tp1Shift=Tp1Shift (any (SetupEngage, 2), : ) ; Tp0Shift=Tp0Shift (any (SetupEngage, 2), : ); FpxShift=FpxShift (any (SetupEngage, 2), : ) ; FpsShift=FpsShift (any (SetupEngage, 2), : ) ; FflyxShift=FflyxShift (any (SetupEngage, 2), : ); FflyShift=FflyShift (any (SetupEngage, 2), :) ; wpcShift=wpcShift (any (SetupEngage, 2), : ); MPMaxShift=MPMaxShift (any (SetupEngage, 2), : ) ;

:Secondary

FxtsShift=FxtsShift (any (SetupEngage,2), : ) ; FytsShift=FytsShift (any (SetupEngage, 2), :); FHelixShift=FHelixShift (any (SetupEngage, 2), : ) ;

FsShift=FsShift (any (SetupEngage, 2), : );

FsxShift=FsxShift (any (SetupEngage, 2), : );

Ts0Shift=Ts0Shift (any (SetupEngage, 2), :);

Ts1Shift=Ts1Shift (any (SetupEngage, 2), :) ;

MSMaxShift=MSMaxShift (any (SetupEngage, 2), : ) ;

EffsShift=EffsShift (any (SetupEngage, 2), : ) ;

$\div$ System

SetupEngage=SetupEngage (any (SetupEngage, 2$)$, : ) ;

Setup=SetupEngage ;

OVehicle speed

wpcLow $=$ zeros $(\operatorname{size}(\operatorname{Setup}, 1), \mathrm{n})$;

VLow $=$ zeros $(\operatorname{size}($ Setup, 1$), \mathrm{n})$;

VShift=zeros (size (Setup, 1$), \mathrm{n})$;

for $i=1: \operatorname{size}($ Setup, 1$)$

wpcLow (i, :)=linspace (wpcEngage (i), wpcShift (i , 1), n) ;

$\operatorname{VLOW}(i,:)=(\operatorname{wpcLow}(i,:) . * \operatorname{Rgb} . * 60 . * 2 . * \mathrm{pi} . * \operatorname{RTir}$ e). / (R (1)*63360);

VShift $(i,:)=(\operatorname{wpcShift}(i,:) . * \operatorname{Rgb} . * 60 . * 2 . *$ pi.* RTire)./(R.*63360);

end

wpc $=[$ wpcLow wpcShift $]$;

$\mathrm{V}=[\mathrm{VLOW}$ VShift];

Rplot=R (1) . *ones $(1, \mathrm{n})$;

Rplot $=[$ Rplot $\mathrm{R}]$;

$\%$ Plotting

응 Engine Speed Vs. Shift Ratio

figure

plot (Rplot', wpc')

grid on 
set (gca,'xdir', 'reverse')

set (gca, 'FontSize', 20)

hold on

oPlot Labels

title('Engine speed Vs. Shift Ratio')

xlabel ('Shift Ratio')

ylabel ('Engine speed [RPM]')

$\operatorname{ylim}\left(\left[\begin{array}{ll}0 & 5000\end{array}\right)\right.$

Reference Markers

yl=yline (Wds, '--', \{'Desired Shift

RPM'\},'fontsize', 20);

yl. LabelVerticalAlignment='middle' ;

yl. LabelHorizontalAlignment=' center' ;

yl=yline (Wde, '--' , \{'Desired Engagement RPM'\}, 'fontsize', 20);

y1. LabelVerticalAlignment='middle',

y1. LabelHorizontalAlignment=' center' ;

$\because$ Reference Lines

$\mathrm{x} 1=\mathrm{xline}(1, \mathrm{\prime}-\mathrm{-}$

',\{'Overdrive'\},'fontsize',20):

x1. LabelVerticalAlignment='middle' ;

xl. LabelHorizontalAlignment=' center' ;

응 Vehicle Speed Vs. Engine Speed

figure

$\operatorname{plot}\left(V^{\prime}, w p c^{\prime}\right)$

grid on

hold on

\%Plot Axes

set (gca, 'FontSize', 20)

title('Engine Speed Vs. Vehicle Speed')

xlabel('Vehicle speed [MPH]')

ylabel ('Engine speed (RPM)')

$y \lim \left(\left[\begin{array}{ll}0 & 5000\end{array}\right)\right.$

$x \lim ([0 \max (V(:, 2 * n))])$

Reference Markers

yl=yline (Wds, '--' ', \{'Desired Shift

RPM'\},' fontsize', 20);

yl. LabelVerticalAlignment=' middle',

y1.LabelHorizontalAlignment=' center' ;

y1=yline (Wde, '--' , \{'Desired Engagement

RPM' \}, 'fontsize', 20);

yl. LabelVerticalAlignment='middle' ;

y1. LabelHorizontalAlignment= ' center' ;

응 Vehicle Speed Vs. Shift Ratio

figure

plot (Rplot, $\left.V^{\prime}\right)$

grid on

set (gca,' 'xdir', 'reverse')

set (gca, 'FontSize',20)

잉t Axes

title('Vehicle speed Vs. Shift Ratio')

xlabel ('Shift Ratio')

ylabel ('Vehicle speed [MPH]')

$x \lim \left(\left[\operatorname{Rplot}\left(2^{*} \mathrm{n}\right)\right.\right.$ 4])

$\because$ Reference Lines

$\mathrm{x} 1=\mathrm{xl}$ ine $(1, \mathrm{\prime}-\mathrm{-}$

',\{'Overdrive'\},'fontsize',20);

x1. LabelVerticalAlignment='middle',

xl. LabelHorizontalAlignment= ' center' ; $\circ$ Primary

응 Torque Received Vs. Maximum Torque

Tranferrable

figure

plot (R, MPMaxShift)

grid on

hold on

ㅇlot Axes

set (gca, 'FontSize', 20)

set (gca, 'xdir', 'reverse')

title('Tranferrable Torque at Primary

Through Shift')

xlabel('Shift Ratio')

ylabel ('Tranferrable Torque (Ft.*lbs)')

$\mathrm{y} \lim \left(\left[\begin{array}{ll}0 & 50\end{array}\right]\right)$

$x \lim ([R(n) R(1)])$

\%Reference Markers

y1=yline (TpShift, '-- ', \{'Minimum

Requirement'\}, 'fontsize', 20$)$

Torque

Ninimum

yl. LabelVerticalAlignment=' middle' ;

yl. LabelHorizontalAlignment= 'center' ;

응 Tension Through Shift

figure

plot (R, Tp0Shift, '-- ', R, Tp1Shift)

grid on

hold on

Plot Axes

set (gca, 'FontSize', 20)

set (gca,'xdir', 'reverse')

title('Belt Tension Through Shift at

Primary')

xlabel('Shift Ratio')

ylabel ('Belt Tension (Lbf)')

$y \lim ([-100200])$

$x \lim ([R(n) R(1)])$

otext Box

str $=\{$ 'Dashed Line: Slack Side

Tension', 'Solid Line: Taught Side Tension'\};

$\operatorname{dim}=\left[\begin{array}{llll}0.7 & 0.6 & 0.3 & 0.3\end{array}\right]$;

t=annotation('textbox', dim, 'String', str, 'Fit

BoxToText', 'on', 'fontsize',20);

\%응 Clamping Force Through Shift

figure

plot (R, FpxShift)

grid on

$\because \mathrm{Plot}$ Axes

set (gca, 'FontSize', 20)

set (gca,'xdir', 'reverse')

title('Total Clamping Force Through Shift at Primary')

xlabel('Shift Ratio')

ylabel('Clamping Force (Lbf)')

$\operatorname{ylim}\left(\left[\begin{array}{ll}0 & 200\end{array}\right]\right)$

$x \lim ([R(n) R(1)])$

$\div$ Secondary

응 Torque Received Vs. Maximum Torque

Tranferrable

figure

plot (R, MSMaxShift)

grid on

hold on 
$\therefore$ Plot Axes

set (gca, 'FontSize', 20)

set (gca,' 'xdir', 'reverse')

title('Tranferrable Torque at Secondary Through Shift')

xlabel ('Shift Ratio')

ylabel('Tranferrable Torque (Ft.*lbs)')

$\operatorname{ylim}\left(\left[\begin{array}{ll}0 & 80\end{array}\right]\right)$

$x \lim ([R(n) R(1)])$

$\therefore$ Reference Markers

plot (R, TsShift, '-- ')

text $\left(2.25,20,{ }^{--}=\right.$Minimum

Requirement','FontSize',20);

\%label (R, 'Minimum

Requirement','slope', 'right')

set (gca, 'FontSize', 20)

응 Tension Through Shift

figure

plot (R, Ts0Shift, '--', R, Ts1Shift)

grid on

hold on

oplot Axes

set (gca, 'FontSize', 20)

set (gca,' 'xdir', 'reverse')

title('Belt Tension Through Shift at

Secondary')

xlabel ('Shift Ratio')

ylabel ('Belt Tension (Lbf)')

$y \lim ([-100200])$

$x \lim ([R(n) R(1)])$

\%Text Box

str $=\{$ 'Dashed Line: Slack Side

Tension','Solid Line: Taught Side Tension'\}; $\operatorname{dim}=\left[\begin{array}{llll}0.7 & 0.6 & 0.3 & 0.3\end{array}\right]$

t=annotation('textbox', dim, 'String', str, 'Fit

BoxToText', 'on' , 'fontsize', 20);

응 Clamping Force Through Shift

figure

plot (R, FsxShift)

grid on

일 Axes

set (gca,' 'FontSize',20)

set (gca,' 'xdir', 'reverse')

title('Total Clamping Force Through Shift at

Secondary')

xlabel ('Shift Ratio')

ylabel ('Clamping Force (Lbf)')

$y \lim \left(\left[\begin{array}{ll}0 & 100\end{array}\right)\right.$

$x \lim ([R(n) R(1)])$

\section{Shift Function:}

function

[TpShift, TsShift, AlphaShift, BetaShift, Tp1Shi ft,Tp0Shift, CpxShift, FpxShift, FpsShift, Fflyx Shift,FflyShift, wpcShift, MPMaxShift, FxtsShif t,FytsShift,FHelixShift,FsShift,FsxShift, Csx Shift, Ts0Shift, TslShift, MSMaxShift, EffsShift , Setupshift $]=$

ShiftV8 ( $\mathrm{n}$, Hds, Wds, Tv, Rp, Rs, R, X1, X2, Y2, U, Uep, Ues, MBelt, BeltSH, Ufly, Cc, Kc, Theta, Phi, Kp, XpO , Delta, Mfly, Rfly, Mlink, Rlink, Xt0, Kt, Yto, Lamb da, Eta, Rr)

ocalculate Setup For Shift
응 Shift Condition

oDesign Power

Hd = Hds; $\quad$ Design power (hp)

\%Angular Velocity

$\mathrm{Wp}=$ Wds; $\quad$ oPrimary angular velocity

(RPM)

Ws $=W p . / R s ; \quad$ oSecondary angular velocity

(RPM)

oTorque at Desired RPM

$\mathrm{Tp}=($ Hd. * 5252) . /Wp; OPrimary Torque

(ft.*Ibs)

$\mathrm{Ts}=\mathrm{Tp} \cdot{ }^{\star} \mathrm{R}$; $\quad$ oTorque at secondary through

shift (ft.*lbs)

응 Belt Calculation

$[\mathrm{Alpha}, \mathrm{Beta}]=\operatorname{BeltV2}(\mathrm{n}, \mathrm{Rp}, \mathrm{Rs}, \mathrm{cc})$;

\% $\frac{\circ}{\circ}$ Preallocation

orimary

Tp1Shift=zeros (length (Kp) *size (Delta, 1) *leng th $(\mathrm{Mfly}) *$ length $(\mathrm{Kt}) *$ length (YtO) * length (Lambd a) * size (Eta, 1), n);

Tp0Shift=zeros (length $(\mathrm{Kp}) * \operatorname{size}($ Delta, 1$)$ *leng th $(\mathrm{Mfly}) *$ length $(\mathrm{Kt})$ *length (YtO) * length (Lambd a) $\left.{ }^{2} \operatorname{size}(\mathrm{Eta}, 1), \mathrm{n}\right)$;

CpxShift=zeros (length (Kp) *size (Delta, 1$)$ *leng th $(\mathrm{Mfly})$ *length $(\mathrm{Kt})$ *length (YtO) * length (Lambd a) * size (Eta, 1), n) ;

FpxShift=zeros (length $(\mathrm{Kp})$ *size (Delta, 1$)$ *leng th $(\mathrm{Mfly}) *$ length $(\mathrm{Kt}) *$ length (YtO) * length (Lambd a) $\left.{ }^{\text {Size }}(\mathrm{Eta}, 1), \mathrm{n}\right)$;

FpsShift=zeros (length $(\mathrm{Kp}) * \operatorname{size}($ Delta, 1$)$ *leng th $(\mathrm{Mfly}) *$ length $(\mathrm{Kt}){ }^{*}$ length (YtO) * length (Lambd a) $\left.{ }^{\operatorname{size}}(\mathrm{Eta}, 1), \mathrm{n}\right)$;

FflyxShift=zeros (length (Kp) *size (Delta, 1) *le ngth $(\mathrm{Mfly})$ *length $(\mathrm{Kt})$ *length (YtO) *length ( Lam bda) * size (Eta, 1), n) ;

FflyShift=zeros (length $(\mathrm{Kp})$ *size $($ Delta, 1$)$ *len gth $(\mathrm{Mfly}) *$ length $(\mathrm{Kt}) *$ length (YtO) * length ( Lamb da) ${ }^{*} \operatorname{size}($ Eta, 1), n)

wpcShift=zeros (length $(\mathrm{Kp}) * \operatorname{size}($ Delta, 1$) *$ leng th $(\mathrm{Mfly}) *$ length $(\mathrm{Kt})$ *length (YtO) * length (Lambd a) $\left.{ }^{\text {size }}(\mathrm{Eta}, 1), \mathrm{n}\right)$;

MPMaxShift=zeros (length $(\mathrm{Kp})$ *size (Delta, 1) *le ngth $(\mathrm{Mfly}) *$ length $(\mathrm{Kt}) *$ length $(\mathrm{YtO}) *$ length (Lam bda) * size (Eta, 1), n) ;

$\because$ Secondary

FxtsShift=zeros (length $(\mathrm{Kp})$ *size $(\operatorname{Delta}, 1) *$ len gth $(\mathrm{Mfly}) *$ length $(\mathrm{Kt}) *$ length (Yto) * length (Lamb da) * size (Eta, 1), n) ;

FytsShift $=$ zeros (length $(\mathrm{Kp}) * \operatorname{size}(\operatorname{Delta}, 1) *$ len gth $(\mathrm{Mfly}) *$ length $(\mathrm{Kt})$ * length (YtO) * length (Lamb da) * size (Eta, 1), n);

FHelixShift=zeros (length $(\mathrm{Kp})$ * size (Delta, 1) *l ength $(\mathrm{Mfly}) *$ length $(\mathrm{Kt}) *$ length (YtO) *length ( La mbda) *size (Eta, 1), n) ;

CsxShift=zeros (length (Kp) *size (Delta, 1$)$ *leng th $(\mathrm{Mfly}) *$ length $(\mathrm{Kt})$ *length (YtO) * length (Lambd a) * size (Eta, 1), n);

FsxShift=zeros (length $(\mathrm{Kp})$ *size $($ Delta, 1$)$ *leng th $(\mathrm{Mfly}) *$ length $(\mathrm{Kt})$ *length (YtO) * length (Lambd a) $\left.{ }^{*} \operatorname{size}(\mathrm{Eta}, 1), \mathrm{n}\right)$;

Ts0Shift=zeros (length (Kp) *size (Delta, 1$)$ *leng th $(\mathrm{Mfly})$ *length $(\mathrm{Kt})$ *length (YtO) * length (Lambd a) $\left.{ }^{*} \operatorname{size}(\mathrm{Eta}, 1), \mathrm{n}\right)$;

Ts1Shift=zeros (length (Kp) *size (Delta, 1$)$ *leng th $(\mathrm{Mfly}) *$ length $(\mathrm{Kt})$ *length (YtO) * length (Lambd a) * size (Eta, 1), n); 
MSMaxShift=zeros (length $(\mathrm{Kp}) * \operatorname{size}(\operatorname{Delta}, 1) * l e$ ngth $(\mathrm{Mfly})$ *length $(\mathrm{Kt}) *$ length (YtO) * length (Lam bda) *size (Eta, 1), n);

EffsShift=zeros (length $(\mathrm{Kp}) * \operatorname{size}(\operatorname{Delta}, 1)$ *len gth $(\mathrm{Mfly}) *$ length $(\mathrm{Kt}) *$ length $($ YtO $) *$ length (Lamb da) *size $(E t a, 1), n)$;

\% System

SetupShift=zeros (length $(\mathrm{Kp}) * \operatorname{size}(\operatorname{Delta}, 1) * l e$ ngth $(\mathrm{Mfly}) *$ length $(\mathrm{Kt})$ *length (YtO) * length (Lam bda) *size (Eta, 1), 7);

응 Primary and Secondary Calculation

$i=0$;

for $A=1$ : length $(\mathrm{Kp})$

for $B=1:$ size (Delta, 1)

for $\mathrm{C}=1$ : length $(\mathrm{Mfly})$

for $D=1$ : length (Kt)

for $\mathrm{E}=1$ : length (Yto)

for $\mathrm{F}=1$ : length (Lambda)

for $G=1$ : size (Eta, 1)

[Fxts, Fyts, FHelix, Fs, Fsx, Csx, Ts0, Ts1, MSMax, E $\mathrm{ffs}]=$

SecondaryV3 ( $\mathrm{n}, \mathrm{MBel}$, Ws, Tv, Ts, X2, Y2, Rs, Phi, Be ta, BeltSH, Ues, Xt0, Kt, Yto, Lambda, Eta, Rr, D, E, F $, \mathrm{G})$;

$[\mathrm{Tp} 1, \mathrm{Tp} 0, \mathrm{Cpx}, \mathrm{Fpx}, \mathrm{Fps}, \mathrm{Fflyx}, \mathrm{Ffly}, \mathrm{wpc}, \mathrm{MPMax}]=$ PrimaryV3 ( $\mathrm{n}, \mathrm{Tp}, \mathrm{MBelt}, \mathrm{Ts} 1$, Wp , X1, Rp, Alpha, Belt $\mathrm{SH}, \mathrm{Uep}, \mathrm{Ufly}, \mathrm{Theta}, \mathrm{Kp}, \mathrm{xp} 0$, Delta, Mfly, Rfly, Mli $\mathrm{nk}$, Rlink, A, B, C) ;

$$
\text { if } w p c>3400 \&
$$

wpc $<3800 \& \operatorname{MPMax}>=0.8 .{ }^{*} \mathrm{Tp} \& \operatorname{MSMax}>=0.8 . * \mathrm{TS}$ $\circ \& \operatorname{MPMax}<=1 \cdot 3 \cdot{ }^{*} \mathrm{TP}$

$i=i+1$

Data Points

Compiling

Primary

Tp1Shift $(i,:)=T p 1$;

Tp0Shift $(i,:)=T p 0$;

CpxShift $(i,:)=C p x$;

FpxShift (i, :)=Fpx;

FpsShift (i, : )=Fps ;

FflyxShift $(i,:)=F f l y x$;

FflyShift $(i,:)=F f l y$;

wpcShift $(i,:)=w p c$;

MPMaxShift $(i,:)=$ MPMax;

․ Secondary

FxtsShift $(i,:)=$ Fxts;

FytsShift $(i,:)=$ Fyts ;

FHelixShift $(i,:)=$ FHelix;

FsShift $(i,:)=F S$;

FsxShift $(i,:)=$ Fsx;

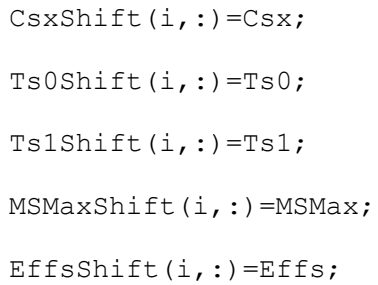

$\%$ Truncate unacceptable combination orimary

Tp1Shift=Tp1Shift (any (Tp1Shift, 2), : ) ; Tp0Shift=Tp0Shift (any (Tp0Shift, 2), : ); CpxShift=CpxShift (any (EpxShift, 2), : ) ; FpxShift=FpxShift (any (FpxShift,2), :) ; FpsShift=FpsShift (any (FpsShift, 2), :) ; FflyxShift=FflyxShift (any (FflyxShift,2), :) ; FflyShift=FflyShift (any (FflyShift,2), : ); wpcShift=wpcShift (any (wpcShift,2), :); MPMaxShift=MPMaxShift (any (MPMaxShift, 2), : ) ;

-Secondary

FxtsShift=FxtsShift (any (FxtsShift,2), : ); FytsShift=FytsShift (any (FytsShift,2), :) ; FHelixShift=FHelixShift (any (FHelixShift, 2$)$, : ) ;

FsShift=FsShift (any (FsShift, 2), : );

FsxShift=FsxShift (any (FsxShift, 2), : );

CsxShift $=$ CsxShift (any (CsxShift, 2), : );

Ts0Shift=Ts0Shift (any (Ts0Shift, 2), :);

Ts1Shift=Ts1Shift (any (TslShift,2), :) ;

MSMaxShift=MSMaxShift (any (MSMaxShift, 2), : ) ; EffsShift=EffsShift (any (EffsShift,2), :) ;

osystem

SetupShift=SetupShift (any (SetupShift,2), : ) ;

\% Renaming

TpShift=Tp;

TsShift=Ts;

AlphaShift=Alpha;

BetaShift=Beta;

end 


\section{Engagement Function:}

\section{function}

[TpEngage, TsEngage, Alpha, Beta, TplEngage, Tp0E ngage, CpxEngage, FpxEngage, FpsEngage, FflyxEng age, FflyEngage, wpcEngage, MPMaxEngage, FxtsEng age, FytsEngage, FHelixEngage, FsxEngage, CsxEng age, Ts0Engage, TslEngage, MSMaxEngage, EffsEnga ge, , SetupEngage $]=$

Engagement ( $n$, SetupShift, Hde, Wde, Tv, Rp, Rs, R, U , Uep, Ues, MBelt, Ufly, cc, Theta, Phi, Kp, Xp0, Delt a, Mfly, Rfly, Mlink, Rlink, Xt0, Kt, Yto, Lambda, Et $\mathrm{a}, \mathrm{Rr})$

oCalculate Setup For Optimal Engagement Point

음 Engagement Condition

$\therefore$ Design Power

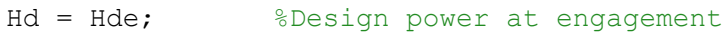

(hp)

$\therefore$ Sheaves

$\mathrm{Rp}=\mathrm{Rp}(1)$;

engagement

$\mathrm{Rs}=\mathrm{Rs}(1)$;

engagement

oPrimary radius during

oSecondary radius during

oAngular Velocity

Wp $=$ Wde; $\quad$ oPrimary angular velocity

(RPM) *Assuming minimal speed differential* Ws=Wp./Rs; $\quad$ osecondary angular velocity (RPM)

$\therefore$ Flyweights

$\operatorname{Rfly}=\operatorname{Rfly}(1)$;

Rlink=Rlink (1);

\%Torque at Desired RPM

$\mathrm{Tp}=($ Hd. *5252).$/ \mathrm{Wp} ; \quad$ orimary Torque

(ft.* ${ }^{*}$ bs)

$\mathrm{Ts}=\mathrm{Tp} \cdot{ }^{\star} \mathrm{R}(1) ; \quad$ oTorque at secondary through

shift (ft.*lbs)

$\therefore$ Preallocation

oPrimary

Tp1Engage=zeros $(\operatorname{size}($ SetupShift, 1$), 1)$; Tp0Engage=zeros ( $\operatorname{size}($ SetupShift, 1$), 1)$; CpxEngage=zeros ( $\operatorname{size}($ SetupShift, 1$), 1)$; FpxEngage=zeros ( $\operatorname{size}($ SetupShift, 1$), 1)$; FpsEngage $=$ zeros $(\operatorname{size}($ SetupShift, 1$), 1)$; FflyxEngage=zeros (size (SetupShift, 1$), 1)$; FflyEngage=zeros ( $\operatorname{size}($ SetupShift, 1$), 1)$; wpcEngage=zeros $($ size $($ SetupShift, 1$), 1)$; MPMaxEngage $=$ zeros $(\operatorname{size}($ SetupShift, 1$), 1)$;

$\div$ Secondary

FxtsEngage $=$ zeros $(\operatorname{size}($ SetupShift, 1$), 1)$; FytsEngage=zeros $(\operatorname{size}($ SetupShift, 1$), 1)$; FHelixEngage=zeros ( $i z e($ SetupShift, 1), 1); CsxEngage $=$ zeros $(\operatorname{size}($ SetupShift, 1$), 1)$; FsxEngage $=$ zeros $($ size $($ SetupShift, 1$), 1)$; Ts0Engage=zeros ( $\operatorname{size}($ SetupShift, 1$), 1)$; TslEngage=zeros $(\operatorname{size}($ SetupShift, 1$), 1)$; MSMaxEngage=zeros ( $\operatorname{size}($ SetupShift, 1$), 1)$; EffsEngage=zeros ( $\operatorname{size}($ SetupShift, 1$), 1)$;

\%System

SetupEngage=zeros ( $\operatorname{size}($ SetupShift, 1$), 7)$; KpEngage=zeros $(1$, size $($ SetupShift, 1$))$;

DeltaEngage $=\operatorname{zeros}(1, \operatorname{size}($ SetupShift, 1$))$; MflyEngage=zeros $(1, \operatorname{size}($ SetupShift, 1$))$;

KtEngage $=$ zeros $(1, \operatorname{size}($ SetupShift, 1$))$;
YtOEngage=zeros $(1, \operatorname{size}($ SetupShift, 1$))$; LambdaEngage=zeros $(1, \operatorname{size}($ SetupShift, 1$))$; EtaEngage=zeros $(1, \operatorname{size}($ SetupShift, 1$))$;

응 Possible Engagement Setups

for $i=1$ :size (SetupShift, 1 ) KpEngage $(i)=\operatorname{Kp}(\operatorname{SetupShift}(i, 1))$ '; DeltaEngage $(i)=\operatorname{Delta}(\operatorname{SetupShift}(i, 2))$ ' ; MflyEngage $(i)=\operatorname{Mfly}(\operatorname{SetupShift}(i, 3))$ '; KtEngage (i) $=$ Kt $($ SetupShift $(i, 4))$ ' ; YtOEngage $(i)=$ Yt0 $($ SetupShift $(i, 5))$ ';

LambdaEngage (i) =Lambda ( $\operatorname{SetupShift~}(i, 6))$ ' ; end EtaEngage $(i)=$ Eta $($ SetupShift $(i, 7))$ ';

$\div$ Belt Calculations Alpha $=(p i-(2 * \operatorname{asin}((\operatorname{Rs}-\mathrm{Rp}) / \mathrm{CC}))) ;$ $\operatorname{Beta}=(p i+(2 * \operatorname{asin}((\mathrm{Rs}-\mathrm{Rp}) / \mathrm{cC})))$;

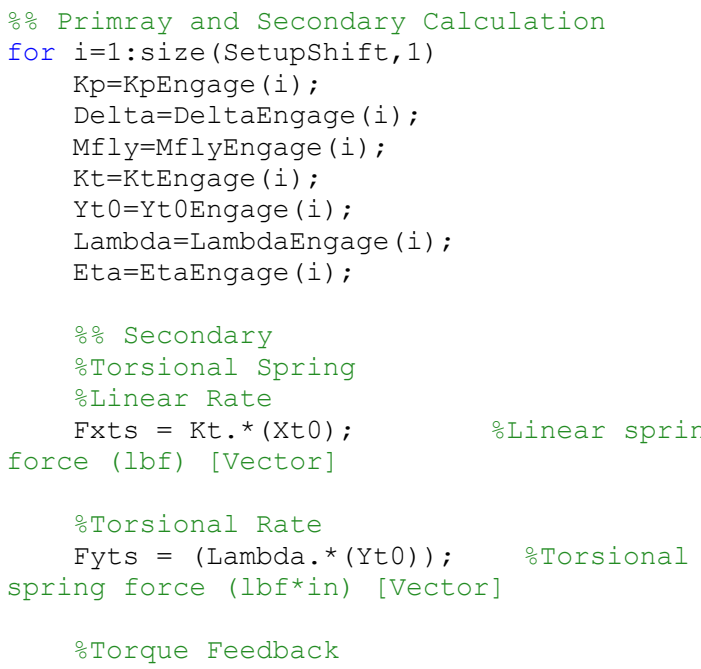


Adjusting For Torque Load from Vehicle Weight

Ts $0=\mathrm{Ts} 0-\left(\mathrm{TV} \cdot{ }^{*} 6\right) \cdot / \mathrm{Rs}$;

$\mathrm{Ts} 1=\mathrm{Ts} 1+(\mathrm{TV} \cdot * 6) \cdot / \mathrm{Rs}$;

\%Maximum Torque Tranferable (Without Slip)

$\operatorname{MSMax}=(\operatorname{Ts} 1-\mathrm{Ts} 0) . *(\mathrm{Rs} . / 12) ; \quad$ \%Maximum torque through shift without slipping

(Ft. * $1 \mathrm{bs})$

\% Secondary Efficiency

Effs $=($ Ts. $/$ MSMax $) . * 100$;

Efficiency into gear box

응 Primary

oTaught side tenion

$\mathrm{Tp} 1=\mathrm{Ts} 1$;

\% Slack side tenion

$\mathrm{Tp} 0=\mathrm{Tp} 1$. / exp (Uep . *Alpha) ;

\%Accounting for Motor Torque

$\mathrm{Tp} 1=\mathrm{Tp} 1+\left(\left(\mathrm{Tp} \cdot{ }^{*} 6\right) \cdot / \mathrm{Rp}\right)$;

$\mathrm{Tp} 0=\mathrm{Tp} 0-((\mathrm{Tp} \cdot * 6) \cdot / \mathrm{Rp})$;

\%Tension Component in X Direction

if Alpha $<=\mathrm{pi}$ $\operatorname{Tp} 0 \mathrm{x}=\mathrm{Tp} 0 .{ }^{\star} \cos (.5 *(\mathrm{pi}-\mathrm{Alpha})) ;$

elseif Alpha>pi $\operatorname{Tp} 0 \mathrm{x}=\mathrm{Tp} 0 .{ }^{\star} \cos (.5 *(\mathrm{Al}$ pha $-\mathrm{pi})) ;$ end

if Alpha $<=p i$ $\operatorname{Tp} 1 \mathrm{x}=\mathrm{Tp} 1 .{ }^{*} \cos (.5 *(\mathrm{pi}-\mathrm{Alpha})) ;$

elseif Alpha>pi end

$\operatorname{Tp} 1 x=T p 1 .{ }^{*} \operatorname{Cos}(.5 *(A l p h a-p i)) ;$

oCentrifugal Force

$\mathrm{Wp}=W \mathrm{p} *(\mathrm{pi} / 30)$;

$\mathrm{Cp}=(1 / 12) \cdot{ }^{\star M B e l t} \cdot{ }^{*}\left(\mathrm{Rp} \cdot{ }^{\wedge} 2\right) \cdot{ }^{\star}\left(\mathrm{Wp} \cdot{ }^{\wedge} 2\right)$;

○Distributed Centrifugal Force

$\mathrm{Cpx}=2 .{ }^{*} \mathrm{Cp} \cdot{ }^{*} \sin (\mathrm{Alpha} \cdot / 2) ; \quad$ \%Total

Centrifugal Force

Required side force

$\mathrm{Fpx}=(-\mathrm{Cpx}) ; \quad$ oTotal clamping force

$\mathrm{Fp}=\left(\mathrm{Fpx} . /\left(2 .{ }^{*} \sin (\mathrm{Alpha} . / 2)\right)\right) . /(2 . *$ tand $($ Theta

/2)); \%Distributed clamping force

Pressure Spring

Fps $=\mathrm{Kp} \cdot{ }^{*}(\mathrm{Xp} 0) ; \quad$ oPressure spring

force vector ( $1 \mathrm{bf})$

\%Required Flyweight force

$\mathrm{Fflyx}=\mathrm{Fp}+\mathrm{Fps}$;

Ffly $=($ Fflyx./tand (Delta) $)-$

$\left(\mathrm{Fflyx} . / \tan \left(\operatorname{acos}\left(\left(\operatorname{Rfl}_{\mathrm{y}}-1.625\right) . / 1.25\right)\right)\right)$;

ㅇequired engine speed

$\mathrm{wpc}=\left(12 \cdot{ }^{\star} \mathrm{Ffly} \cdot /\left(\left(\mathrm{Mfly} \cdot{ }^{\star} \mathrm{Rfly}\right)+\left(\mathrm{Mlink} \cdot{ }^{\star} \mathrm{Rlink}\right)\right)\right.$ ).$\wedge .5$;

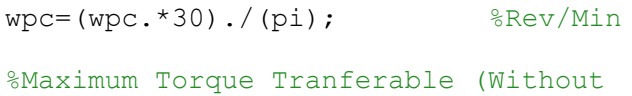


Ts1Engage=Ts1Engage (any (Ts1Engage, 2$),:)$; MSMaxEngage=MSMaxEngage (any (MSMaxEngage, 2 ) , : );

EffsEngage=EffsEngage (any (EffsEngage, 2), : ) ;

응 Renaming

TpEngage $=\mathrm{Tp}$;

TsEngage $=$ Ts ;

AlphaEngage $=$ Alpha;

BetaEngage=Beta;

end

\section{Belt Function:}

function [Alpha, Beta] $=\operatorname{BeltV} 2(\mathrm{n}, \mathrm{Rp}, \mathrm{Rs}, \mathrm{CC})$

oCalculates angle of wrap at primary and

secondary through shift

oBelt Wrap

$\mathrm{A}=\mathrm{RS}>=\mathrm{Rp} ; \quad \quad$ DDetermine when secondary

has larger working radius than primary

$\mathrm{A}=\mathrm{A}(:, \operatorname{any}(\mathrm{A}, 1))$;

opreallocation

Alpha=zeros $(1, n) ; \quad \frac{0}{0}$ Primary belt wrap

through shift (Degrees)

Beta=zeros $(1, n) ; \quad$ osecondary belt wrap

through shift (Degrees)

for $i=1$ : length $(A)$

Alpha $(i)=(p i-(2 * a s i n($ Rs $(i)-$

$\operatorname{Rp}(i)) /(C)$ )) );

$\operatorname{Beta}(i)=(p i+(2 * \operatorname{asin}((\operatorname{Rs}(i)-$

$\operatorname{Rp}(i)) / C C)$ )) ;

end

for $i=($ length $(A)+1): n$

Alpha $(i)=(p i+(2 * \operatorname{asin}((R p(i)-$

Rs (i))/CC))!;

$\operatorname{Beta}(i)=(p i-(2 * \operatorname{asin}((\operatorname{Rp}(i)-$

Rs(i))/CC)) );

end

end

\section{Primary Function:}

function

$[T p 1, T p 0, C p x, F p x, F p s, F f l y x, F f l y, w p c, M P M a x]=$ PrimaryV3 ( $n, T p$, MBelt, Ts1,Wp, X1, Rp, Alpha, Belt $\mathrm{SH}, \mathrm{Uep}, \mathrm{Ufly}$, Theta, Kp, Xpo, Delta, Mfly, Rfly, Mli nk, Rlink, A, B, C)

oCalculate Primary RPM

oTaught side tenion

$\mathrm{Tp} 1=\mathrm{Ts} 1$;

Slack side tenion

$\operatorname{Tp} 0=\operatorname{Tp} 1 . / \exp ($ Uep. $*$ Alpha $)$;

oAccounting for Motor Torque

$\mathrm{Tp} 1=\mathrm{Tp} 1+((\mathrm{Tp} \cdot * 6) \cdot / \mathrm{Rp})$;

$\mathrm{Tp} 0=\mathrm{Tp} 0-((\mathrm{Tp} \cdot * 6) \cdot / \mathrm{Rp})$;

oTension Component in $\mathrm{X}$ Direction

$\operatorname{Tp} 0 \mathrm{x}=\operatorname{zeros}(1, \mathrm{n})$;

for $i=1: 1: n$

if Alpha (i) $<=$ pi

Alpha (i)))

$\operatorname{Tp} 0 x(i)=\operatorname{Tp} 0(i) \cdot{ }^{\star} \cos (.5 *(\mathrm{pi}-$

elseif Alpha(i)>pi

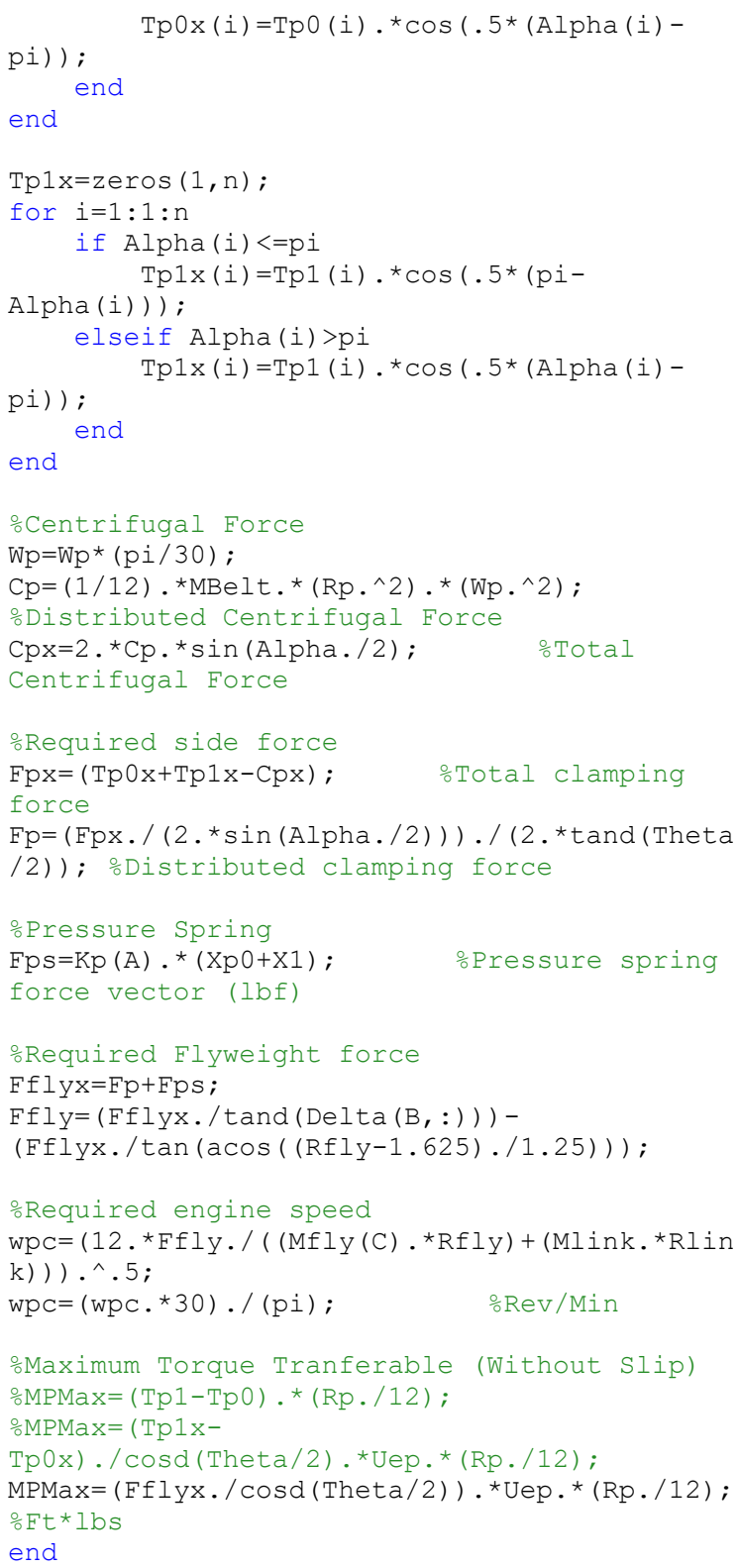

\section{Secondary Function:}

function

[Fxts, Fyts, FHelix, Fs, Fsx, Csx, Ts0, Ts1, MSMax, E ffs] $=$

SecondaryV3 ( $\mathrm{n}, \mathrm{MBelt}, \mathrm{Ws}, \mathrm{TV}, \mathrm{Ts}, \mathrm{X} 2, \mathrm{Y} 2, \mathrm{Rs}, \mathrm{Ph}$, $\mathrm{Be}$ ta, BeltSH, Ues, Xt0, Kt, Yto, Lambda, Eta, Rr, D, E, F $, \mathrm{G})$

oCalculate Secondary Belt Tenion

oTorsional Spring

oLinear Rate

Fxts $=$ Kt $(\mathrm{D}) \cdot{ }^{*}(\mathrm{Xt} 0+\mathrm{X} 2) ; \quad$ oLinear

spring force (lbf) [Vector]

oTorsional Rate

Fyts $=\left(\operatorname{Lambda}(\mathrm{F}) \cdot{ }^{*}(\mathrm{YtO}(\mathrm{E})+\mathrm{Y} 2)\right)$

oTorsional spring force (lbf*in) [Vector] 


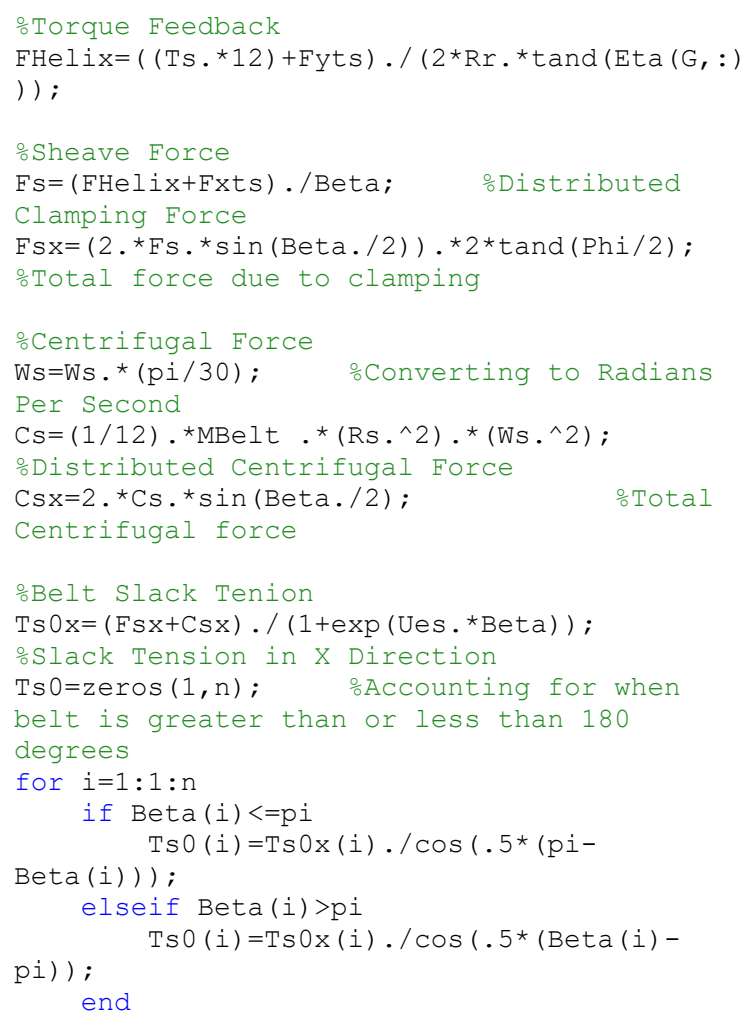

end

oTaught Side Tenion

Ts $1=$ Ts $0 .{ }^{*} \exp ($ Ues. *Beta) ;

\%Adjusting For Torque Load from Vehicle Weight

$\operatorname{Ts} 0=\mathrm{Ts} 0-(\mathrm{TV} \cdot * 6) \cdot / \mathrm{Rs}$;

$\mathrm{Ts} 1=\mathrm{Ts} 1+\left(\mathrm{TV} \cdot{ }^{*} 6\right) \cdot / \mathrm{Rs}$;

oMaximum Torque Tranferable (Without Slip)

$\circ \mathrm{MSMax}=(\mathrm{FSX} \cdot / \mathrm{Cosd}(\mathrm{Phi} / 2)) . * \mathrm{Ues} \cdot{ }^{*}(\mathrm{RS} \cdot / 12)$; $\operatorname{MSMax}=(\operatorname{Ts} 1-\mathrm{Ts} 0) . *(\mathrm{Rs} . / 12) ; \quad$ oMaximum torque through shift without slipping (Ft. * $1 \mathrm{bs})$

\% Secondary Efficiency EffS $=(($ MSMax-TS $) . /$ MSMax $) . * 100$;

\% Secondary Efficiency into gear box

end 